\title{
Adaptive Estimation of Acoustic Normal Modes
}

by

\author{
Kathleen E. Wage
}

B.S., Electrical and Computer Engineering, University of Tennessee (1990)

Submitted in partial fulfillment

of the requirements for the degree of

\begin{tabular}{|c|c}
\hline $\begin{array}{c}\text { MARINE } \\
\text { BIOCOGICAL } \\
\text { LAOORATORY }\end{array}$ & $\begin{array}{c}\text { Master of Science } \\
\text { at the }\end{array}$ \\
\hline LISRARY & MASSACHUSETTS INSTITUTE OF TECHNOLOGY \\
\hline $\begin{array}{c}\text { WOUS HOLE, MASS. } \\
\text { W. H. O. I. }\end{array}$ & WOODS HOLE OCEANOGRAPHIC INSTITUTION
\end{tabular}

September 1994

(C)Massachusetts Institute of Technology and Woods Hole Oceanographic Institution, 1994

All rights reserved.

Author

MIT Department of Electrical Engineering and Computer Science/ WHOI Department of Applied Ocean Science and Engineering

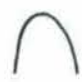

September 9, 1994

Certified by

Arthur B. Baggeroer

Ford Professor of Electrical and Ocean Engineering

Accepted by

Arthur B. Baggeroer

Chairman, MIT/WHOI Joint Committee for Applied Ocean Science and Engineering

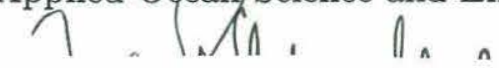

Accepted by

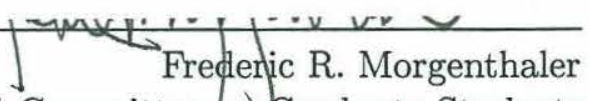

Chairman, MIT Electrical Engineering Departmental Committee on Graduate Students

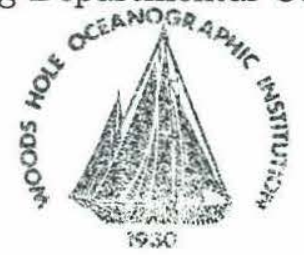





\title{
Adaptive Estimation of Acoustic Normal Modes
}

\author{
by
}

Kathleen E. Wage

\begin{abstract}
Submitted to the MITT Department of Electrical Engineering and Computer Science and to the WHOI Department of Applied Ocean Science and Engineering

in partial fulfillment of the requirements for the degree of

Master of Science
\end{abstract}

\begin{abstract}
Normal mode theory provides an efficient description of signals which propagate axially in the SOFAR channel and are detectable at long ranges. Mode amplitudes and their second order statistics are useful in studies of long-range acoustic propagation and for applications such as Matched Mode Processing (MMP) and Matched Field Tomography (MFT). The purpose of this research is to investigate techniques for estimating the average power in the modes of a signal given pressure measurements from a vertical line array.

This thesis develops the problem of mode estimation within a general array processing framework which includes both deterministic and stochastic characterizations of the modal structure. A review of conventional modal beamforming indicates that these methods provide poor resolution in low signal-to-noise ratio environments. This is not surprising since standard estimation techniques rely on minimizing a squared error criterion without regard to the ambient noise. The primary contribution of this thesis is an adaptive estimator for coherent modes that is based on a method suggested by Ferrara and Parks for array processing using diversely-polarized antennas. Two formulations of the adaptive method are investigated using a combination of analytical techniques and numerical simulations. The performance evaluation considers the following issues: (i) power level of the noise, (ii) orthogonality of the sampled modeshapes, (iii) number of data snapshots, and (iv) coherence of the signal. The new approach is fundamentally different from other modal estimators such as those used in MMP because it is data-adaptive and maximizes the received power instead of minimizing the squared error. As a result, the new methods perform significantly better than least squares in high noise environments. Specifically, the Ferrara/Parks formulations are able to maintain nulls in the modal spectrum since they do not suffer the bias error that significantly affects the least squares processor.

A second contribution of the thesis is an extension of the coherent estimator to facilitate estimation of phase-randomized modes. Although the results of this work are preliminary, the extended formulation appears to offer several advantages over least squares in certain cases.
\end{abstract}

Thesis Supervisor: Arthur B. Baggeroer

Title: Ford Professor of Electrical and Ocean Engineering 


\section{Acknowledgements}

First, I thank God for the many blessings that have enriched my life and ultimately made this work possible.

I thank my advisor, Prof. Arthur Baggeroer, for suggesting this area of research and for providing invaluable technical guidance along the way. Thanks also to Brian Sperry for many useful discussions regarding this research.

I have learned a great deal from my colleagues in the Digital Signal Processing Group. Their help and friendship is much appreciated. In particular, I am indebted to Steve Isabelle for his thoughtful advice and encouragement.

I consider my experiences as a teaching assistant to be one of the most valuable aspects of the past three years. It has been a true privilege to work with John Buck, Prof. Steve Leeb, Prof. Alan Oppenheim, Stephen Scherock, and Andy Singer.

Many people have provided the necessary doses of humor, encouragement, and e-mail that have helped me maintain my sanity in graduate school. For their kindness and friendship I am especially grateful to: Amy Troutman, Emily Parkany, Dr. Cristi Bell-Huff, Melissa Caldwell, Aradhana Narula, Karin Knoll, Dr. Ellen Livingston, Mark Allen, and Susie Wee. A special thanks to the Knoxville-based moral support engineering firm of "Dr." Kathy and Steve Drevik for their southern hospitality.

At this stage of my life, no thesis cannot be complete without acknowledging all of the people who got married while I was working on it. So a special thanks (and best wishes!) to the following couples who provided me with some welcome weekends away from MIT: Amanda and Randy, Kathy and Steve, Pam and David, Lizette and Frank, Becky and Mike, Karen and Randy, Cristi and John, Melissa and Eric, and Traci and Ernesto.

Finally, I am eternally grateful to my parents, Jim and Virginia Wage, for their love and support. It is with sincere thanks that I dedicate this thesis to them.

Funding for this work was provided by a Clare Boothe Luce Fellowship (1991-1992) and the University of California - Scripps ATOC Agreement, PO\#10037359. 


\section{Contents}

$\begin{array}{ll}\text { Abstract } & 3\end{array}$

$\begin{array}{ll}\text { Acknowledgements } & 4\end{array}$

Table of Contents $\quad 5$

$\begin{array}{ll}\text { List of Figures } & 7\end{array}$

1 Introduction $\quad 10$

1.1 Motivation .............................. 10

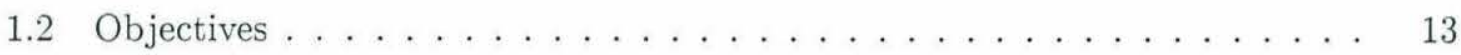

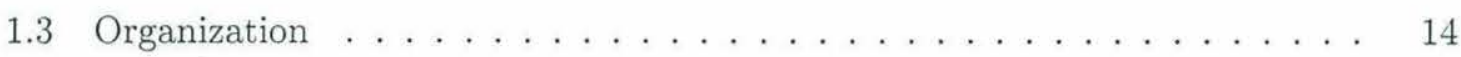

2 Background $\quad 15$

2.1 Ocean Acoustic Waveguide . . . . . . . . . . . . . . . 15

2.2 Normal Mode Representation of Narrowband Signals . . . . . . . . . . . 17

2.2.1 Modal Propagation in a Range-Independent Waveguide . . . . . . 18

2.2.2 Modal Propagation in a Range-Dependent Waveguide . . . . . . . 22

2.2.3 Modal Propagation in a Random Waveguide . . . . . . . . . . . 23

2.3 Problem Formulation . . . . . . . . . . . . . . . . . . . . . . . . . . . . . . 24

2.4 Important Considerations in Modal Array Processing . . . . . . . . . . . 26

2.4 Ambient Noise . . . . . . . . . . . . . . . 27

2.4 .2 Modal Orthogonality . . . . . . . . . . . . . . 28

2.4 .3 Estimation of the Covariance Matrix . . . . . . . . . . . . . 30

2.4 .4 Signal Coherence . . . . . . . . . . . . . . . . . . . . . 31

2.4 .5 Performance Measures . . . . . . . . . . . . . . . . . . 32 


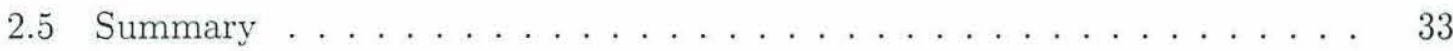

3 Least Squares Methods $\quad 34$

3.1 Standard Least Squares . . . . . . . . . . . . . . . . . . . . . . 35

$3.1 .1 \quad$ Ambient Noise . . . . . . . . . . . . . . . . . . . . . 39

3.1 .2 Modal Orthogonality . . . . . . . . . . . . . . . . . 41

3.1.3 Estimated Covariance Issues . . . . . . . . . . . . . . . . . . . . . . 44

3.1 .4 Signal Coherence . . . . . . . . . . . . . . . . . . . . . . 45

3.2 Summary . . . . . . . . . . . . . . . . . . . 45

4 Adaptive Estimation of Coherent Modes $\quad 47$

4.1 Minimum Variance Modal Estimator . . . . . . . . . . . . . . . . . 49

4.1 .1 Ambient Noise . . . . . . . . . . . . . . . . 55

4.1 .2 Modal Orthogonality . . . . . . . . . . . . . . . . . . . 57

$4.1 .3 \quad$ Estimated Covariance Issues . . . . . . . . . . . . . . . . . . 59

4.1 .4 Signal Coherence . . . . . . . . . . . . . . . . . . . . 63

4.2 MUSIC Modal Estimator . . . . . . . . . . . . . . . . . 65

4.2 .1 Ambient Noise . . . . . . . . . . . . . . . . . . . 71

4.2 .2 Modal Orthogonality . . . . . . . . . . . . . . . . 73

$4.2 .3 \quad$ Estimated Covariance Issues . . . . . . . . . . . . . . . . 74

4.2 .4 Signal Coherence . . . . . . . . . . . . . . . . . 76

4.3 Summary . . . . . . . . . . . . . . . . . . . . . 77

5 Adaptive Estimation of Incoherent Modes $\quad 79$

5.1 Modified Minimum Variance Formulation . . . . . . . . . . . . . . . . . 79

5.2 Summary . . . . . . . . . . . . . . . . . . 86

$\begin{array}{llr}6 & \text { Conclusion } & 88\end{array}$

6.1 Summary . . . . . . . . . . . . . . . . . . . . . . . 88

6.2 Suggestions for Further Research . . . . . . . . . . . . . . . . . . . . . 89

A Fisher Information Matrix for Modal Estimation $\quad 91$

B Mean and Variance of the Least Squares Power Estimates 93 
C Mean and Variance of the MV Power Estimates

D Mean and Variance of the MUSIC Power Estimates

Bibliography

99 


\section{List of Figures}

2-1 Model of an ocean environment . . . . . . . . . . . . . . . . 16

2-2 Data acquisition and pre-processing . . . . . . . . . . . . 17

2-3 Idealized range-independent waveguide . . . . . . . . . . . . . . 18

2-4 Sound speed profile and modeshapes for the deep water waveguide . . . . 20

$2-5$ Simulation array for the deep water waveguide $\ldots \ldots \ldots \ldots$

2-6 Modeshape correlation for the deep water waveguide . . . . . . . . . 30

2-7 Degrees of freedom for the simulation array . . . . . . . . . . . . 31

3-1 Least squares power estimates for the deep water example . . . . . . . . . 37

3-2 Error in least squares power estimates for the deep water example . . . . . 39

3-3 Error vs. SNR for modes 1, 2, and 10 of the deep water waveguide. Ambient noise consists of white sensor noise only. . . . . . . . . . . . . . . 40

3-4 Error vector for the 16-mode example using the deep water waveguide . . . 42

3-5 Total error vs. number of modes to estimate for the deep water example . . 43

3-6 Bias error vs. number of snapshots for mode 1. Predicted and Monte Carlo results for the LS estimator are shown. . . . . . . . . . . . . . . . 45

3-7 Variance vs. number of snapshots for mode 1. Predicted and Monte Carlo results for the LS estimator are shown along with the Cramer Rao bound. . 46

4-1 Minimum variance power estimates for the deep water example . . . . . . . 52

4-2 Error in minimum variance power estimates for the deep water example . . 55

4-3 Error vs. SNR for modes 1, 2, and 10 of the deep water waveguide. Ambient noise consists of white sensor noise only. Both MV and LS errors are shown for the ideal covariance case. Note that the vertical scale for mode 10 is different than for modes 1 and $2 \ldots \ldots \ldots \ldots$. . . . . . . . . 56 
4-4 Error vector for the 16-mode example using the deep water waveguide . . .

4-5 Total error vs. number of modes to estimate for the deep water example. Two cases are shown: the top plot displays the $0 \mathrm{~dB}$ SNR results and the bottom plot displays the $-10 \mathrm{~dB}$ SNR results. . . . . . . . . . . . . . 58

4-6 Example of global localization problem for the MV estimator: 15 modes estimated at $-10 \mathrm{~dB}$ SNR. . . . . . . . . . . . . . . . 6 60

4-7 Peaks of the minimum variance power spectrum for the 15 -mode estimate at two SNR levels: $0 \mathrm{~dB}$ and $-10 \mathrm{~dB}$. . . . . . . . . . . . . . . . . 60

4-8 Bias error vs. number of snapshots for mode 1. Predicted and Monte Carlo results are shown. . . . . . . . . . . . . . .

4-9 Variance v's. number of snapshots for the MV estimator. Predicted and Monte Carlo simulation values are shown. The Cramer Rao bound is given for reference. . . . . . . . . . . . . . . . 63

4-10 Total error vs. coherence for the Ferrara/Parks MV formulation . . . . . . . 64

4-11 MIUSIC power estimates for the deep water example . . . . . . . . . . . . 69

4-12 Error in MUSIC power estimates for the deep water example . . . . . . . 71

4-13 Error vs. SNR for modes 1, 2, and 10 of the deep water waveguide. Ambient noise consists of white sensor noise only. MUSIC, MV, and LS error curves are shown for the ideal covariance case. Note that the vertical scale for mode 10 differs from the one used for modes 1 and $2 \ldots \ldots \ldots$. . . . . . .

4-14 Error vector for the 16-mode example using the deep water waveguide. ML$\mathrm{SIC}, \mathrm{MV}$, and LS results are shown. . . . . . . . . . . .

4-15 Total error vs. number of modes to estimate for the deep water example. Results for two input SNR's $(0 \mathrm{~dB}$ and $-10 \mathrm{~dB})$ are shown. . . . . . . . . . 74

4-16 Error vs. number of snapshots for mode 1. Predicted and Monte Carlo results are shown for all three estimators. . . . . . . . . . . . 76

4-17 Tariance vs. number of snapshots for the MUSIC estimator. . . . . . . . . 77

4-18 Total error vs. coherence for the Ferrara/Parks MUSIC formulation . . . . . 78

5-1 Estimation Error for Partially Incoherent Mode Example . . . . . . . . . 82

5-2 Total Error vs. Coherence for the Modified MV Algorithm. SNR=0 dB. . . 85

5-3 Total Error vs. Coherence for the Modified MV Algorithm. SNR=10 dB. . 86 


\section{Chapter 1}

\section{Introduction}

\subsection{Motivation}

The ocean is an extremely efficient channel which is capable of transmitting low frequency sound over long distances. Sound waves refract towards regions of lower velocity, therefore a minimum in sound speed creates an acoustic channel. For example, sound propagating downward from a source bends back toward the channel axis (depth of minimum sound speed). Once it passes through the axis on an upward path, it begins to bend away from the surface. In this fashion, sound is effectively trapped in a duct and can propagate from a source to a receiver hundreds or even thousands of kilometers away. In the deep ocean a minimum sound speed (occurring at approximately $1 \mathrm{~km}$ below the surface) defines the ocean acoustic waveguide which is also known as the SOund Fixing And Ranging (SOFAR) channel [1].

Normal mode theory provides an efficient description of low frequency sound traveling along refracted paths in the SOFAR channel. Coherent interference of a family of rays that share the same phase speed creates a standing wave (or mode) in depth. The modes form a complete set of orthogonal basis functions in the vertical. As a result, the sound pressure at any receiver point in the waveguide consists of a weighted sum of normal modes. The phase speed specifies the turning depths of the rays and determines the vertical extent of each corresponding mode. High order modes have low phase speeds and are associated with families of rays that intersect the surface or bottom boundaries. Since these modes suffer severe losses, the lower order or axial modes contain most of the signal energy at long ranges. The normal mode representation is efficient because only a subset of modes are 
required to describe a signal at a significant distance from its source.

Normal mode theory offers more than just a convenient description of acoustic signals. Since the modes span different vertical sections of the water column, they each provide specific information about the sound source and the propagation path. Consequently, the normal mode decomposition is useful in several applications such as source localization and acoustic tomography. For example, recent papers indicate that source range and depth information may be obtained through matched field beamforming using the modal coefficients (or weights) $[2,3,4]$. In the literature this technique is known as Matched Mode Processing (MMP). ${ }^{1}$ In another application, acoustic tomography is used to measure the speed of sound in the ocean. Normal modes are useful in this regard since modal group delays depend on the sound speed. Provided the mode arrivals can be reliably tracked, researchers can obtain estimates of the sound speed. In turn, these estimates provide valuable information about the channel. Note that one of the fundamental problems associated with both of the aforementioned applications is the detection and estimation of the modes in an acoustic signal. The purpose of this research is to explore techniques for modal estimation.

Receivers capable of extracting the underlying modal structure typically consist of vertical arrays of sensors. One characteristic of acoustic propagation is that modes travel with different group velocities. Note that the group delays associated with the higher order modes may permit resolution on the basis of arrival time. ${ }^{2}$ Temporal resolution of the lower order modes, however, is not usually possible since their group delays are almost identical. Instead, an array can resolve these axial modes based on differences in their spatial distributions. This thesis focuses on array processing algorithms for estimation of the low order modes because they remain the most energetic at long ranges from the source.

The success of the applications described above depends on accurate modeling of acoustic propagation. Many issues concerning transmissions over long distances in the ocean remain unresolved. Two of these issues, mode coherence and mode coupling, are particularly relevant to this thesis since they affect the mode estimation problem.

Mode coherence refers to the relative phasing between the modes of an acoustic signal. Coherent propagation means that the modes remain phase-locked as they travel. Incoherent propagation implies that the phase fluctuations in the signal vary from mode to mode.

\footnotetext{
${ }^{1}$ Baggeroer et. al. provide a comprehensive review of matched field techniques [5].

${ }^{2}$ In this case it is usually easier to track the ray arrivals associated with the higher order modes.
} 
Although coherent signal models appear to be fairly accurate over moderate distances, recent experimental evidence suggests that transmission over megameter ranges results in incoherent signals [6]. More experiments are required before accurate predictions of signal coherence are possible. Since the characteristics of a channel may not be known a priori, it is important to analyze the effects of coherence on modal estimation algorithms.

Mode coupling is the second major issue to consider. Models for slowly range-varying environments typically assume that the propagation is adiabatic, therefore no energy is transferred between modes. This simplifying assumption is not realistic over long ranges. Travel time analysis using modal arrivals requires a knowledge of the coupling characteristics. In practice it is often necessary to measure the coupling by examining the modal content of signals from a known source. The need for this type of experiment motivates the development of high resolution mode estimation algorithms.

This thesis considers mode estimation in a general context, but it is useful to mention a specific practical application in order to highlight several important aspects of the problem. Over the past several years, researchers have endeavored to exploit the efficiency of the ocean waveguide to study global environmental change. The fundamental idea behind these experiments is that changes in water temperature may be inferred from changes in the travel time of acoustic signals since the speed of sound depends on temperature. Because of the large local variability inherent in the ocean environment, this type of acoustic tomography requires long transmission paths in order to obtain reliable measurements of average water temperature. Accurate estimation of travel times and subsequent inversions for temperature depend on a thorough understanding of acoustic propagation over ranges on the order of 10,000 to $20,000 \mathrm{~km}$ (10-20 megameters). Two experiments have been designed to study global-scale propagation [7]. The first of these is the Heard Island Feasibility Test (HIFT) which took place over a 5-day period in January of 1991. HIFT demonstrated that coded low frequency signals can be received at megameter distances from a source. The Acoustic Thermometry of Ocean Climate (ATOC) project is a follow-up to Heard Island which is intended to show that the techniques developed with HIFT can be extended to study propagation paths and characteristics over several seasons. Both projects have emphasized the importance of using vertical line arrays (VLA's) to resolve the modal structure of received signals.

The nature of long-range propagation imposes some design requirements on the array 
processing for the VLA's. First of all, the algorithms must be able to extract the modal arrivals from a relatively high noise background. Low signal-to-noise ratios are unavoidable in global acoustics research because of restricted source levels and large transmission losses. Environmental impact concerns and technical limitations constrain the source power output. Also, transmission losses over ranges of 10 to 20 megameters are quite significant. A second design requirement is that the estimators provide an accurate indication of which modes are truly present in the received signal. Obviously this is always a desirable property, but it is especially important for global experiments since adequate models for propagation over megameter distances do not exist. For example, if the output of the array processing is to be used to study mode coupling, then it is essential that nulls in the modal power distribution be maintained.

This section has indicated the importance of the normal mode decomposition for acoustic signals, thereby motivating the study of array processing methods for modal estimation. An example has been given to illustrate some of the relevant design constraints. The next section briefly reviews conventional methods and outlines the objectives of this thesis.

\subsection{Objectives}

The relative distribution of power among the modes of a signal provides valuable insights about mode coherence and coupling and is useful in detecting the modal arrivals for time delay estimation. Consequently, the goal of this thesis is to estimate the modal power spectrum given a set of measurements from a vertical line array.

Recent research in this area has concentrated on modal beamforming algorithms which produce time series of modal amplitudes $[8,9]$. These algorithms, which have been developed primarily for MMP applications, rely on least squares estimation theory. Least squares methods minimize a squared error criterion without regard to the noise contained in the signal. Naturally, a strategy that ignores noise components is not expected to perform well in low SNR environments. This motivates the search for a fundamentally new approach to mode estimation.

This thesis has three primary objectives:

1. To develop the modal estimation problem within a general array processing framework which includes both deterministic and stochastic characterizations of the modal 
structure.

2. To formulate a fundamentally new approach to the modal estimation problem.

3. To evaluate the performance of the new algorithms with respect to conventional estimation techniques.

Four criteria are used in evaluating the new approach: (i) power level of the noise, (ii) orthogonality of the sampled modeshapes, (iii) number of data snapshots, and (iv) coherence of the signal.

\subsection{Organization}

The remainder of this thesis consists of five chapters. Chapter 2 develops relevant background material and formulates the modal estimation problem. In addition, the simulation environment used for the numerical examples throughout the thesis is described. Chapter 3 reviews the conventional approach to modal analysis. Chapter 4 develops a new estimator for coherent modes and analyzes it with respect to the criteria mentioned above. Two formulations of the new method are considered, and a set of numerical examples are used to draw comparisons between the new and the conventional approaches. Chapter 5 presents an extension of the coherent mode estimation algorithm to the more general case of random

or incoherent modes. Finally, Chapter 6 provides a summary and indicates future directions for research. 


\section{Chapter 2}

\section{Background}

The purpose of this chapter is to define clearly the acoustic mode estimation problem within an array processing framework. The first section describes a basic model of an ocean waveguide which contains an acoustic source and a set of receivers. Section 2.2 reviews the normal mode representation of signals in both range-independent and range-varying waveguides. This section is intended as a brief overview of modal propagation. More comprehensive treatments of normal mode theory are found in the classic text by Brekhovskikh and Lysanov [10] and in more recent books by Frisk [11] and Jensen, et. al. [12]. Section 2.3 formulates the general mode estimation problem and highlights the importance of two special cases: (i) coherent modes and (ii) incoherent modes. The remainder of the chapter discusses important issues related to the signal processing and introduces performance measures for modal estimation algorithms. Over the course of the chapter, a deep water simulation environment is developed. This waveguide is used for the numerical examples throughout the thesis. ${ }^{1}$

\subsection{Ocean Acoustic Waveguide}

Figure 2-1 depicts a model of an ocean environment containing a narrowband source, several types of noise sources, and a receiving array. The density and sound speed profiles along with a set of boundary conditions for the surface, sediment and basement layer interfaces specify

\footnotetext{
${ }^{1}$ For the purposes of this thesis, scalar quantities are denoted by italics, column vectors by lowercase bold letters and matrices by uppercase bold letters. The superscript $\dagger$ represents the complex conjugate transpose operation and $\mathcal{E}$ is the expected value operator.
} 
the propagation characteristics of the medium. A cylindrical coordinate system with range $r$ and depth (positive-downward) $z$ describes the waveguide. For the purposes of this thesis, the environment is assumed to be independent of azimuthal angle. The model shown in the figure is range-invariant, but this is obviously not true for real ocean waveguides. Acoustic propagation using both range-independent and range-dependent models is discussed below.

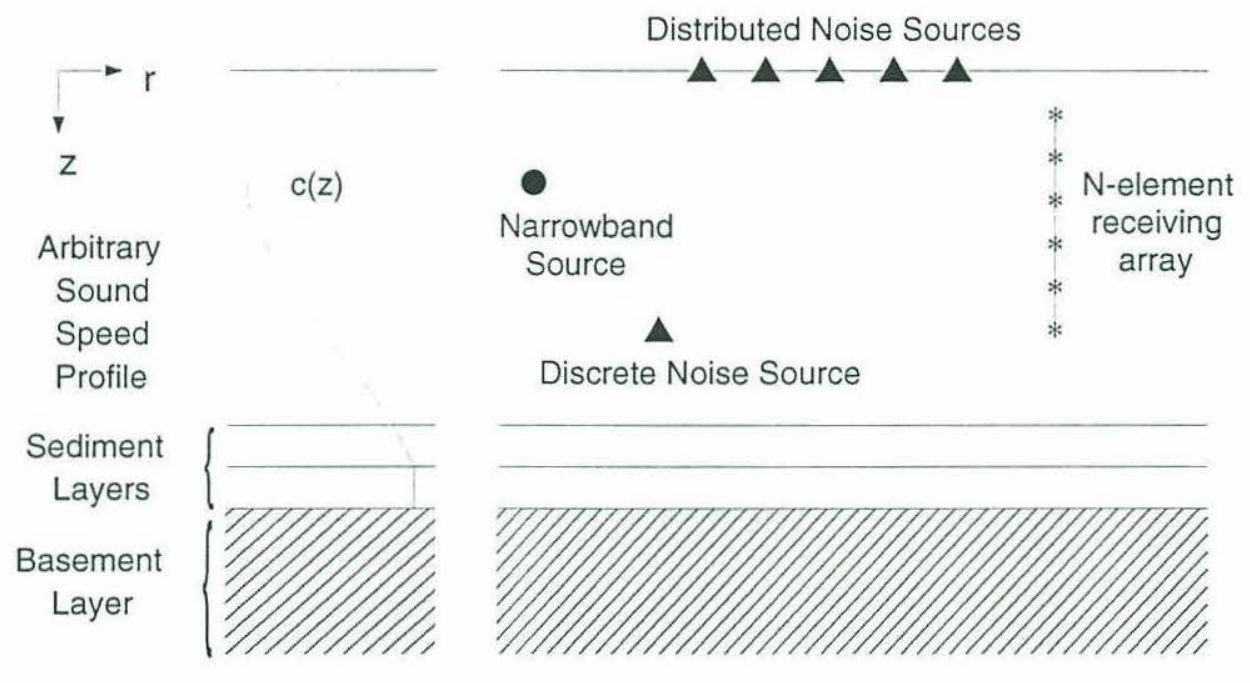

Figure 2-1: Model of an ocean environment

The source is a narrowband point source operating at a frequency $\omega$. Propagation studies typically use low frequency tonal sources because higher frequencies are attenuated rapidly by the ocean's intrinsic absorptive processes. Several noise sources are indicated in the figure. Surface noise sources generate spatially correlated noise that has a structure which is strongly influenced by the propagation environment. Discrete noise sources are modeled as narrowband point sources with temporal and spatial characteristics similar to that of the signal source. The spatial structure of the noise is explored in more detail in Section 2.4.

The receiving array and the associated data acquisition system are responsible for temporal and spatial sampling of the ambient wave field. Vertical deployment, as shown in Figure 2-1, is common for mode resolving arrays. Figure 2-2 shows a typical data acquisition and pre-processing system. Standard pre-processing of the antenna outputs consists of temporal sampling followed by demodulation at the frequency of the source. The result is a vector time series, $\mathrm{p}(l)$, of quadrature components representing the pressure field. 
Most processors include filters to improve input signal-to-noise ratio. The rest of this thesis implicitly assumes that these basic pre-processing steps have been taken.

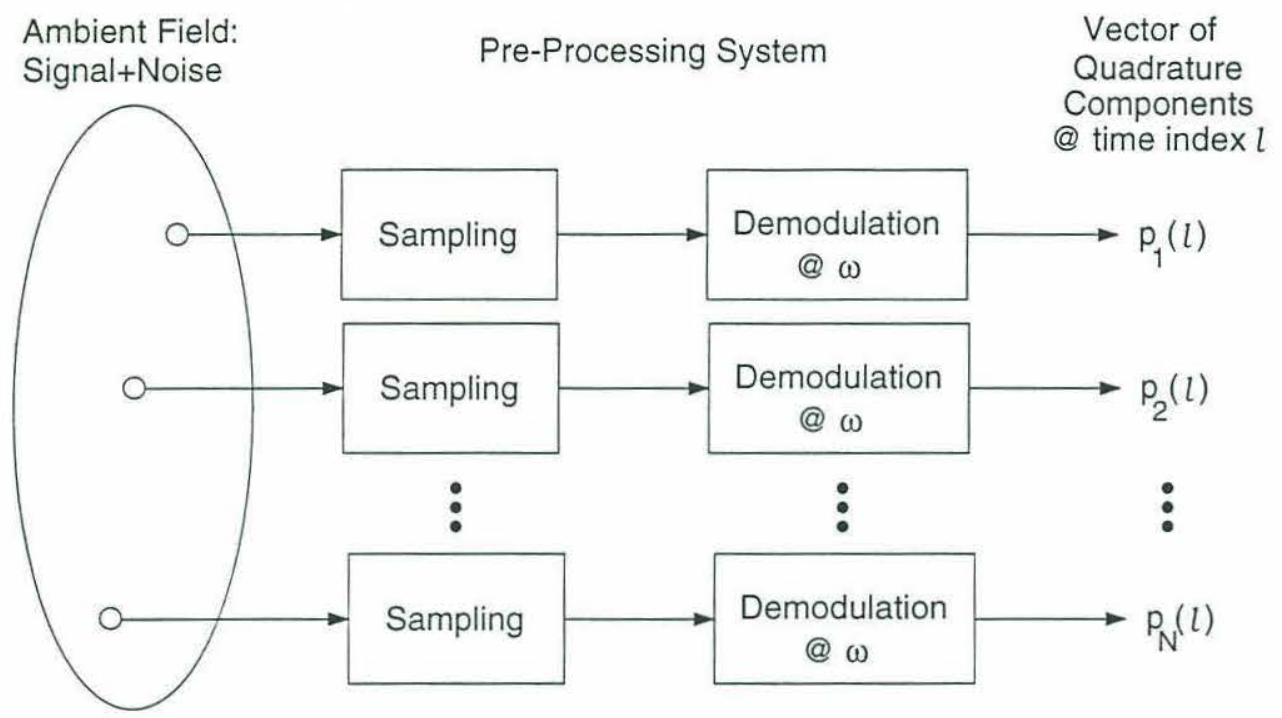

Figure 2-2: Data acquisition and pre-processing

The ocean model used in this research consists of a horizontally-stratified medium with arbitrary sound speed and density profiles in the vertical which is bounded from above and below by semi-infinite halfspaces. The upper halfspace above the water's surface is modeled as a vacuum, and the lower halfspace has characteristics similar to the sediment layers. Range dependencies may be incorporated into the model by propagating signals through a cascade of range-independent sections.

\subsection{Normal Mode Representation of Narrowband Signals}

The acoustic wave equation, with parameters and boundary conditions derived from the environment, describes the propagation of sound in a horizontally-stratified waveguide. The sound pressure field generated by a narrowband source is conveniently characterized by Fourier transforming the wave equation to obtain the Helmholtz equation. Normal mode solutions to the Helmholtz equation are the focus of this section. The frequency dependence $(\omega=2 \pi f)$ of the narrowband signal is suppressed in the following development. 


\subsubsection{Modal Propagation in a Range-Independent Waveguide}

In this section the environment is assumed to be range-independent and is characterized by the sound speed profile $c(z)$ and the density profile $\rho(z)$ which vary with depth. Let $p(r, z)$ be the sound pressure for a narrowband source and the Helmholtz equation becomes

$$
\frac{1}{r} \frac{d}{d r}\left(r \frac{d p}{d r}\right)+\rho(z)\left(\frac{1}{\rho(z)} \frac{d p}{d z}\right)+k^{2}(z) p(r, z)=F(r, z) \quad k^{2}(z)=\frac{\omega^{2}}{c^{2}(z)}
$$

where $k$ is the wavenumber associated with the medium. $F(r, z)$ is the forcing function associated with the acoustic point source. Consider the idealized range-independent waveguide shown in Figure 2-3. The waveguide is of depth $H$ with a pressure release boundary at the surface $(z=0)$ and a perfectly rigid boundary at the bottom $(z=H)$. An arbitrary depthdependent sound speed profile is assumed. The rigid (non-propagating) bottom boundary condition simplifies this initial development; a brief discussion of the implications of more realistic bottom conditions follows.

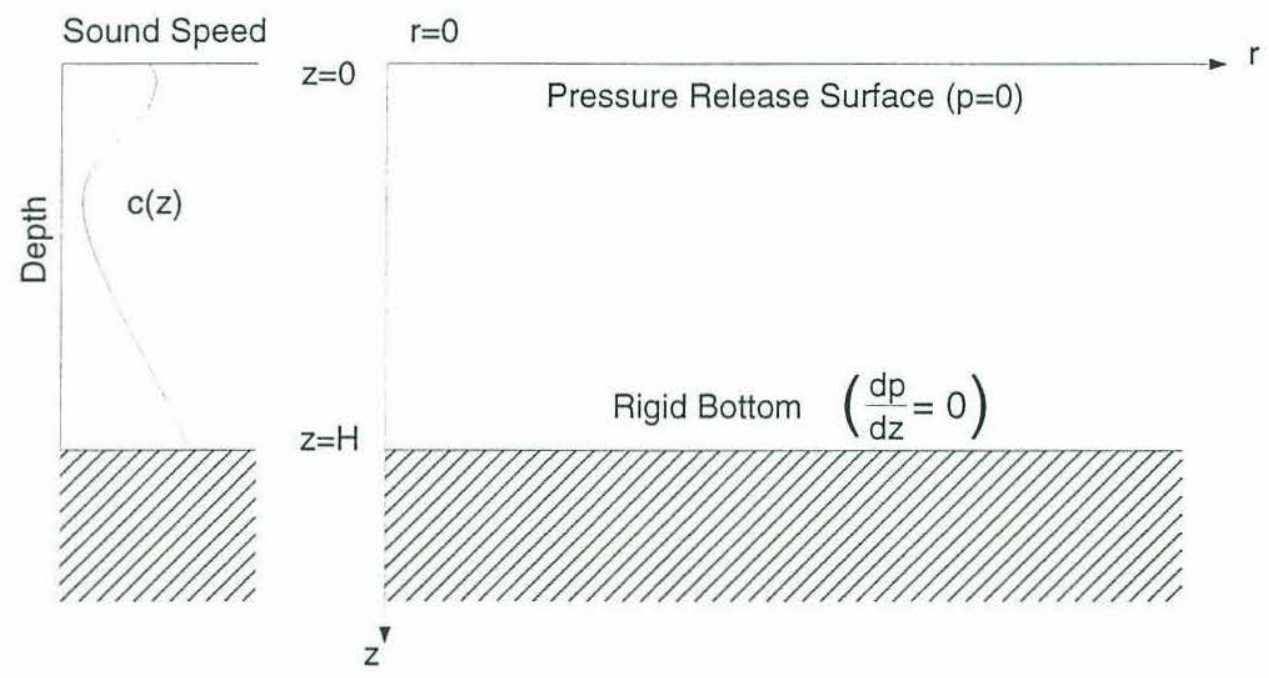

Figure 2-3: Idealized range-independent waveguide

The separation of variables technique yields solutions to the unforced $(F=0)$ Helmholtz equation of the form [10]

$$
p(r, z)=H_{0}^{(1)}\left(k_{m} r\right) \phi_{m}(z)
$$

The range-dependent portion of the solution, $H_{0}^{(1)}\left(k_{m} r\right)$, is a zero-order Hankel function of 
the first kind. The depth function $\phi_{m}$ satisfies the following eigenvalue problem

$$
\frac{d^{2} \phi_{m}}{d z^{2}}+\left[k^{2}(z)-k_{m}^{2}\right] \phi_{m}=0
$$

For the simple boundary conditions described above, this equation is a classical SturmLiouville problem where the depth functions (modes) form a complete orthonormal (CON) set. Thus, the solution to Equation 2.1 for a point source consists of a weighted sum of the normal modes

$$
p(r, z)=\sum_{m} w_{m} H_{0}^{(1)}\left(k_{m} r\right) \phi_{m}(z) .
$$

When the forcing function $F$ is known, the orthogonality of the modes may be exploited to determine the weights, $w_{m}$. In the case of a unit-normalized point source at depth $z_{s}$ and range $r=0$, the pressure at depth $z$ and range $r$ becomes

$$
p(r, z)=\frac{i}{\rho\left(z_{s}\right) \sqrt{8 \pi r}} e^{-i \pi / 4} \sum_{m} \phi_{m}\left(z_{s}\right) \phi_{m}(z) \frac{e^{i k_{m} r}}{\sqrt{k_{m}}}
$$

An asymptotic approximation for the Hankel function has been used to derive this result. As shown in Equation 2.5 the source excites each mode at a level proportional to the value of the mode function $\phi_{m}$ at the source depth $z_{s}$. While the sum is infinite, only a finite number of modes actually propagate in the waveguide. Higher than a certain mode number $m$, the horizontal wavenumbers are imaginary, therefore contributions from these modes are exponentially decaying with range. Modes with imaginary $k_{m}$ are called evanescent and do not affect the modal sum if the point of interest is at any significant distance from the source.

Although the essential features of the normal mode decomposition are revealed in the idealized waveguide example, practical models require more realistic bottom conditions. Jensen et. al. [12] provide a clear generalized derivation which incorporates arbitrary bottom boundary conditions. The additional mathematical rigor offers few new insights, however. Suffice it to say that at long ranges away from the source, the signal may always be written as a weighted sum of the propagating modes.

Consider the following example of a realistic waveguide. This simulation environment is used for all of the numerical examples in the later chapters. 


\section{Deep Water Simulation Environment}

The channel is 4000 meters deep and is characterized by a Munk profile [13]. This profile is a canonical deep water sound speed profile with a single minimum. In this case the minimum sound speed is $1480 \mathrm{~m} / \mathrm{s}$ at an axis depth of 1000 meters. Water density is assumed to be $1.0 \mathrm{~g} / \mathrm{cm}^{3}$. The left side of Figure $2-4$ shows the sound speed profile for the simulation environment. A $70 \mathrm{~Hz}$ narrowband source is used for all the numerical examples. The right

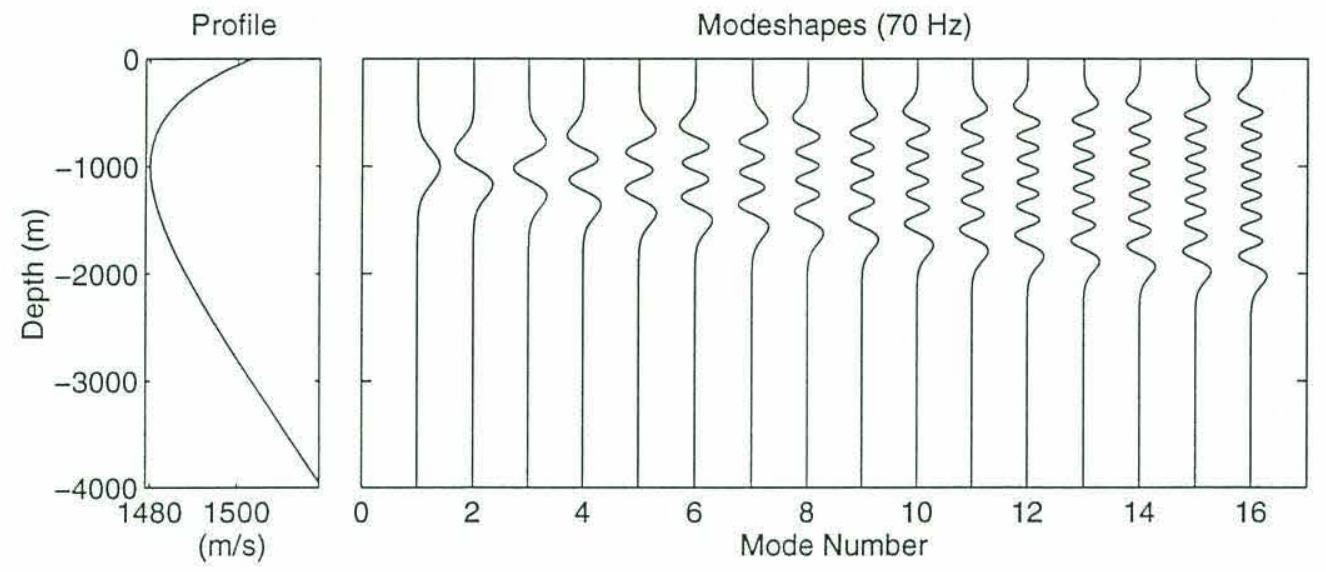

Figure 2-4: Sound speed profile and modeshapes for the deep water waveguide

side of Figure 2-4 is a plot of the modeshapes for the first 16 modes associated with the source. Note that the vertical extent of these modes effectively defines a channel in which low-angle rays can propagate outward from a source on the axis. The modeshapes were computed using a normal mode code developed by Baggeroer [14].

Consider sampling the wave field generated by a point source using a receiving array. Assuming that the field is composed of a subset of $M$ discrete modes, the sum in Equation 2.5 is most conveniently written using linear algebra notation. The vector of pressures measured by an $N$-element vertical array is defined as follows

$$
\mathrm{p}=\tilde{b} \mathbf{E P x}
$$

where

- $\tilde{b}$ is a complex Gaussian random variable that models the phase uncertainty inherent 
in the signal processing,

$$
\mathcal{E}\{\tilde{b}\}=0 \quad \mathcal{E}\left\{|\tilde{b}|^{2}\right\}=\sigma_{b}^{2}
$$

- E is an $N \times M$ matrix of sampled modeshapes,

$$
\mathrm{E}=\left[\begin{array}{cccc}
\phi_{1}\left(z_{1}\right) & \phi_{2}\left(z_{1}\right) & \cdots & \phi_{M}\left(z_{1}\right) \\
\phi_{1}\left(z_{2}\right) & \phi_{2}\left(z_{2}\right) & \cdots & \phi_{M}\left(z_{2}\right) \\
\vdots & \vdots & & \vdots \\
\phi_{1}\left(z_{N}\right) & \phi_{2}\left(z_{N}\right) & \cdots & \phi_{M}\left(z_{N}\right)
\end{array}\right]
$$

- $\mathrm{P}$ is an $M \times M$ diagonal propagation matrix,

$$
\mathbf{P}=\frac{i}{\rho\left(z_{s}\right) \sqrt{8 \pi}} e^{-i \pi / 4}\left[\begin{array}{ccc}
\ddots & 0 & 0 \\
0 & \frac{e^{i k_{m} r}}{\sqrt{k_{m} r}} & 0 \\
0 & 0 & \ddots
\end{array}\right]
$$

- $\mathrm{x}$ is a vector of mode depth amplitudes containing the source excitation,

$$
\mathrm{x}=\left[\begin{array}{c}
\phi_{1}\left(z_{s}\right) \\
\phi_{2}\left(z_{s}\right) \\
\vdots \\
\phi_{M}\left(z_{s}\right)
\end{array}\right]
$$

Equation 2.6 provides a compact representation of modal propagation in a range-independent waveguide. The matrix $\mathrm{P}$ transforms the initial excitation of the modes at the source (represented by $\mathrm{x}$ ) to the excitation levels at the receiver. The diagonal structure of the propagation matrix $\mathbf{P}$ reflects the fact that there is no transfer of energy among the modes. Note that the matrix $\mathbf{E}$ requires a slight modification to account for phase difference across the array if all sensors are not at the same range, i.e., if the array is tilted. The inclusion of $\tilde{b}$ in the model implies that the received pressures are contained in a zero-mean Gaussian random vector. 


\subsubsection{Modal Propagation in a Range-Dependent Waveguide}

As previously indicated, models of range-dependent channels typically consist of a cascade of range-independent sections. A partial separation of variables solution leads to a set of mode depth functions and horizontal wavenumbers for each section. Regardless of the range-dependence of the waveguide, the signal at a receiving array may be written as a weighted sum of the "local" modes, i.e.,

$$
\mathrm{p}=\tilde{b} \mathrm{EPx}
$$

where $\mathbf{E}$ is a matrix of sampled modeshapes for the segment containing the receiver. As defined in Section 2.2.1, $\tilde{b}$ and $\mathrm{x}$ represent the inherent phase uncertainty and the initial modal excitation, respectively. Modifications to the matrix $\mathrm{P}$ account for the range dependent nature of the propagation.

In general, range dependencies in a channel lead to transfers of energy among the modes. As a result of this coupling, $\mathbf{P}$ is no longer a diagonal matrix. Full coupled mode theory usually requires numerical solution of the range equations for each segment in order to obtain the coupling parameters.

For mildly range-dependent environments, however, the adiabatic approximation leads to an analytically-tractable range solution. Adiabatic normal mode theory assumes that the range dependence is gradual enough that an individual propagating mode adapts with range but does not transfer energy into the other modes. In other words, the modeshapes and wavenumbers vary with range, but the modes do not couple or scatter into each other. The adiabatic approach results in the following summation for the pressure at a single receiver

$$
p(r, z)=\frac{i}{\rho\left(z_{s}\right) \sqrt{8 \pi}} e^{-i \pi / 4} \sum_{m} \psi_{m}\left(z_{s}\right) \phi_{m}(z) \frac{e^{i \overline{k_{m}} r}}{\sqrt{\overline{k_{m}} r}}
$$

where the range-averaged wavenumber is defined as

$$
\overline{k_{m}}=\frac{1}{r} \int_{0}^{r} k_{m}\left(r^{\prime}\right) d r^{\prime},
$$

and $\psi_{m}(z)$ and $\phi_{m}(z)$ represent the modeshapes at the source and receiver locations, respectively. The $k_{m}\left(r^{\prime}\right)$ are the horizontal wavenumbers for each range segment in the channel. 
For an adiabatic model, the $\mathbf{P}$ matrix is the same as in Equation 2.9 provided that the range-averaged wavenumber $\overline{k_{m}}$ is substituted for $k_{m}$.

\subsubsection{Modal Propagation in a Random Waveguide}

The models discussed in the previous sections assume that the ocean is a deterministic medium, however experimental evidence indicates the presence of internal wave fields which can perturb the local sound speed profile. These perturbations introduce fluctuations in acoustic signals which can be simulated using stochastic propagation models. The literature contains many references to wave propagation in random media. In particular, Dozier and Tappert have presented a statistical theory of modal propagation in a random ocean $[15,16]$. Baggeroer and Kuperman propose a paradigm for matched field processing in a stochastic channel [17].

The framework of Equation 2.6 still applies for a random ocean, provided that the definitions of $\tilde{b}$ and $\mathbf{P}$ are modified accordingly. The propagation term $\mathbf{P}$ becomes a matrix of zero-mean Gaussian random variables. The zero-mean and Gaussian assumptions again reflect the phase uncertainty inherent in the signal processing. The source scaling term, $\tilde{b}$, is a constant that is retained for consistency with the models described in the two previous sections $\left(|\tilde{b}|^{2}=\sigma_{b}^{2}\right)$. For the random case a channel is specified in terms of the second order statistics of the modal excitation, i.e., $\mathcal{E}\left\{\mathrm{Pxx}^{\dagger} \mathrm{P}^{\dagger}\right\}$.

Baggeroer and Kuperman offer several examples of random channels [17]. The one that is relevant for later examples in this thesis corresponds to an adiabatic channel with internal waves. For this channel the second order statistics are shown below

$$
\mathcal{E}\left\{\mathrm{Pxx}^{\dagger} \mathrm{P}^{\dagger}\right\}=(1-\gamma) \operatorname{diag}\left[\mathrm{x}_{0} \mathrm{x}_{0}^{\dagger}\right]+\gamma \mathrm{x}_{0} \mathrm{x}_{0}^{\dagger} \quad \text { where } \quad 0 \leq \gamma \leq 1
$$

The operator diag indicates that only the diagonal terms are used; the off-diagonals are set to zero. The vector $\mathrm{x}_{0}$ represents the modal amplitude at the receiver for a deterministic adiabatic channel. Coherence of the signal is determined by the parameter $\gamma$. When $\gamma$ is equal to 1 , the modes are phase locked; the propagation characteristics correspond to those of a deterministic adiabatic channel. At the other extreme, when $\gamma$ is equal to 0 , the modes are phase random; this implies a totally incoherent signal. The structure in Equation 2.13 ensures that there is no energy exchanged among the modes, hence the adiabatic assumption 
is satisfied.

\subsection{Problem Formulation}

As indicated in Chapter 1, the average power in the normal modes provides valuable information about the propagation environment. The objective of the algorithms discussed in this thesis is to estimate the power in each mode given a set of pressure measurements from an array of sensors. Since high order modes are less energetic at long ranges and can be resolved temporally, subsequent discussions focus on estimating the power spectrum of the low order modes.

Regardless of the characteristics of the waveguide, the signal measured by a receiver may be written as a weighted sum of the local modes

$$
\mathrm{p}=\mathrm{Ea}
$$

where $\mathrm{E}$ is a matrix of local modeshapes and $\mathrm{a}$ is a vector of coefficients associated with the modes. The vector a contains the relative levels of each mode, as determined by the initial source excitation and the propagation characteristics of the medium, i.e.,

$$
\mathrm{a}=\tilde{b} \mathrm{Px}
$$

From the definitions of $\tilde{b}, \mathrm{P}$, and $\mathrm{x}$ used in each of the previous sections, $\mathrm{a}$ is a zero-mean, Gaussian random vector with the $M \times M$ correlation matrix $\mathrm{S}_{\mathrm{M}}$ defined below,

$$
\mathrm{S}_{\mathrm{M}}=\mathcal{E}\left\{\mathrm{aa}^{\dagger}\right\}=\mathcal{E}\left\{|\tilde{b}|^{2} \mathrm{Pxx}^{\dagger} \mathrm{P}^{\dagger}\right\}=\sigma_{b}^{2} \mathcal{E}\left\{\mathrm{Pxx}^{\dagger} \mathrm{P}^{\dagger}\right\}
$$

The diagonal terms of $\mathbf{S}_{\mathrm{M}}$ are the average powers in the modes and the off-diagonal terms indicate correlation among the modes. Clearly the propagation environment, represented by $\mathrm{P}$, determines the structure of the mode correlation matrix. In general $\mathrm{S}_{\mathrm{M}}$ is an arbitrary positive semi-definite $M \times M$ matrix, but two special cases are worth mentioning.

\section{Coherent Modes}

Recall from Chapter 1 that a signal is coherent when phase variations affecting one mode 
do not occur independently of phase variations for any other mode. Deterministic, timeinvariant channels are always coherent; random or time-varying channels may or may not be coherent depending on the statistics of the propagation matrix P. Most matched field processing (MFP) algorithms rely on coherent signal models to generate replica vectors. Coherency is a reasonable assumption for transmission over moderate distances within an ocean basin. For example, Polcari has shown that the Arctic Ocean is a stable, highly coherent channel [9].

When the modes are perfectly correlated, the $\mathbf{S}_{\mathrm{M}}$ matrix has rank 1 (only one non-zero eigenvalue). As a result, two parameters completely specify the correlation structure, i.e.,

$$
\mathrm{S}_{\mathrm{M}}=\mathcal{P}_{\mathrm{T}}\left[\mathbf{a}_{\mathrm{T}} \mathbf{a}_{\mathrm{T}}^{\dagger}\right]
$$

$\mathcal{P}_{\mathrm{T}}$ represents the total power in the signal and $\mathrm{a}_{\mathrm{T}}$ is a normalized $\left(\mathrm{a}_{\mathrm{T}}^{\dagger} \mathrm{a}_{\mathrm{T}}=1\right)$ vector containing the relative modal power distribution. Note that $\mathcal{P}_{\mathrm{T}}$ is the non-zero eigenvalue of $\mathbf{S}_{\mathrm{M}}$ and $\mathrm{a}_{\mathrm{T}}$ is the corresponding eigenvector. This formulation is useful in analyzing the coherent mode estimators developed in Chapter 4.

\section{Incoherent Modes}

A signal is totally incoherent when the modes are phase random with respect to one another. For example, data taken with one of the HIFT vertical line arrays indicates that a signal which was transmitted over an $18,000 \mathrm{~km}$ path consisted of an incoherent sum of modes [6]. Phase coherence of the normal modes is an aspect of global propation that is not well-understood. Signal randomization is more often considered in the context of rays. Theory predicts that signals traveling along different ray paths are uncorrelated at long ranges. Brekhovskikh and Lysanov [10] provide a useful discussion of this topic.

Incoherent modes are uncorrelated, thus $\mathrm{S}_{\mathrm{M}}$ is a diagonal matrix,

$$
\mathrm{S}_{\mathrm{M}}=\sigma_{b}^{2}\left[\begin{array}{cccc}
\mathcal{E}\left(a_{1}^{2}\right) & 0 & \cdots & 0 \\
0 & \mathcal{E}\left(a_{2}^{2}\right) & \ddots & \vdots \\
\vdots & \ddots & \ddots & 0 \\
0 & \cdots & 0 & \mathcal{E}\left(a_{M}^{2}\right)
\end{array}\right]
$$

Note that for an incoherent signal containing $M$ modes, the mode correlation matrix has 
$\operatorname{rank} M$.

The goal of this thesis is to investigate new methods for estimating the modal power distribution from sound pressure measurements made with a vertical line array. In a realistic ocean environment, the measured pressure consists of the modal signal plus noise

$$
\mathrm{p}=\mathrm{Ea}+\mathrm{n}
$$

The signal and the noise are independent vector random processes, therefore a statistical description of the received field is useful. The modal signal is a zero-mean process with covarinace

$$
\mathrm{S}_{\mathrm{S}}=\mathcal{E}\left\{\mathrm{Eaa}^{\dagger} \mathrm{E}^{\dagger}\right\}=\mathrm{ES}_{\mathrm{M}} \mathrm{E}^{\dagger}
$$

Ambient noise in the waveguide is assumed to be zero-mean with covariance $\mathbf{S}_{N}$. The structure of $\mathbf{S}_{N}$ is discussed in Section 2.4.1. The above assumptions imply that $\mathrm{p}$ is a zero-mean random vector with the covariance given below

$$
\mathrm{S}=\mathrm{S}_{\mathrm{S}}+\mathrm{S}_{\mathrm{N}}
$$

$\mathrm{S}$ is an $N \times N$ matrix referred to as the sensor covariance matrix. The algorithms described in the remaining chapters attempt to extract the average modal powers (diagonal terms of $\mathrm{S}_{\mathrm{M}}$ ) from the pressure field characterized by $\mathrm{S}$. In the case of a partially incoherent or random channel, the off-diagonal of $\mathrm{S}_{\mathrm{M}}$ terms contain valuable information, but a thorough study of their estimation is beyond the scope of this thesis.

\subsection{Important Considerations in Modal Array Processing}

The purpose of this section is to highlight important signal processing issues that arise in using vertical line arrays to sample modal fields. Later chapters characterize the performance of estimators in terms of the four criteria discussed below. 


\subsubsection{Ambient Noise}

Ambient noise in the waveguide is described by $\mathrm{S}_{\mathrm{N}}$, the noise covariance among the array elements at a specific frequency. Ocean noise can be divided into three general categories:

1. Sensor noise: Spatially-correlated noise with the covariance matrix $\mathbf{S}_{W}=\sigma_{w}^{2} \mathbf{I}$ where $\mathbf{I}$ is the identity matrix. The sensor noise level $\sigma_{w}^{2}$ may depend on the source frequency.

2. Distributed noise: Correlated noise with the covariance matrix $\mathbf{S}_{C}$ whose structure is determined by the propagation environment. One example of distributed noise is surface-generated noise.

3. Discrete noise sources: Noise sources that have signal-like qualities and contribute to the far-field effects that are described by the discrete modes. The covariance of discrete noise is denoted by

$$
\mathrm{S}_{D}=\mathrm{Edd}^{\dagger} \mathbf{E}^{\dagger}
$$

where $\mathrm{d}$ is the vector of mode amplitudes for the discrete noise source.

The total noise covariance is

$$
\mathrm{S}_{\mathrm{N}}=\mathrm{S}_{W}+\mathrm{S}_{C}+\mathrm{S}_{D}
$$

Since the propagation environment is inhomogeneous in the vertical, the signal-to-noise ratio (SNR) differs from sensor to sensor on the array. It is sometimes convenient to define the SNR at the input to an $N$-element array as the geometric mean of the SNR's at each array element i.e.,

$$
S N R=\left[\left(S N R_{1}\right)\left(S N R_{2}\right) \cdots\left(S N R_{N}\right)\right]^{\frac{1}{N}}
$$

or in $\mathrm{dB}$

$$
S N R=\frac{1}{N} \sum_{n=1}^{N} 10 \log _{10}\left(S N R_{n}\right)
$$

In a homogeneous environment where the SNR is identical at all elements, the above equation reduces to

$$
S N R=10 \log _{10}\left(S N R_{n}\right) .
$$

Chapter 1 notes the prevalence of low SNR environments in global propagation studies. For example, input SNR's (before pre-processing) for the HIFT Monterey vertical line array were approximately -10 to $-15 \mathrm{~dB}$ on a single hydrophone [18]. Since low SNR's can adversely 
affect performance, it is important to consider the impact of noise on mode estimation algorithms.

\subsubsection{Modal Orthogonality}

The second major issue in modal estimation concerns the array's ability to sample the pressure field. In principle, a filled array which spans the water column can resolve a complete set of orthonormal modes. In practice however, arrays consist of discrete elements spanning a limited aperture. As a result, the sampled mode shapes may not be orthogonal, even though the true modes form a CON set. Realistically, an array can spatially resolve only that finite set of modes which are adequately sampled by its sensors.

One way of measuring the orthogonality of the sampled modes is to examine the modeshape correlation matrix $\mathrm{E}^{\dagger} \mathrm{E}$. If the sampled modes are orthogonal, this matrix is diagonal. (For the purpose of this thesis, it is convenient to assume that the modeshapes are scaled

equally such that orthogonal modes correspond to an $\mathrm{E}^{\dagger} \mathrm{E}$ matrix that is a mulitple of the identity matrix. This assumption simplifies bookeeping somewhat, but is not a necessary condition for orthogonality.) If the sampled modes are not orthogonal, the matrix contains non-zero off-diagonal terms which represent the "cross-talk" between the modes. As an example, consider sampling the deep water environment with an array. The array geometry described below is used for numerical examples in the rest of the thesis.

\section{Simulation Array}

The array consists of 40 elements with 35 meter sensor spacing and spans almost 1400 meters. Figure 2-5 shows the first 10 modeshapes in relation to the position of the array. The top sensor is located at a depth of $475 \mathrm{~m}$, resulting in 40 percent of the elements being positioned above the channel axis. The array is designed to adequately sample the first 10 modes.

Figure 2-6 is a plot of the modeshape correlation using the first 16 modes of the waveguide for the $70 \mathrm{~Hz}$ frequency band. The plot shows the elements of the correlation matrix on a $\log$ scale $\left(10 \log _{10} \mathrm{E}^{\dagger} \mathrm{E}\right)$, normalized such that the maximum element corresponds to $0 \mathrm{~dB}$. Note that above mode 11 , the off-diagonal terms become significant. This is expected since the array is designed to sample the lowest 10 modes.

One useful scalar measure of orthogonality is the effective degrees of freedom of the 


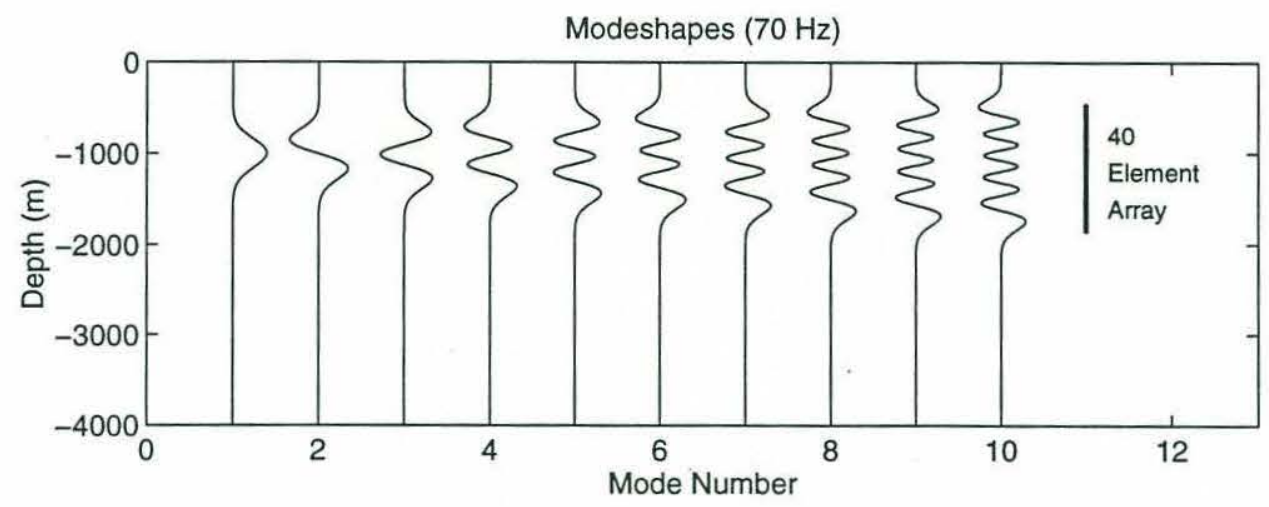

Figure 2-5: Simulation array for the deep water waveguide

modeshape correlation matrix, defined as

$$
D O F_{\text {eff }}=\frac{\left(\sum_{i=1}^{M} \mu_{i}\right)^{2}}{\sum_{i=1}^{M} \mu_{i}^{2}}
$$

where the $\mu_{i}$ are the eigenvalues of $\mathbf{E}^{\dagger} \mathbf{E}$. $D O F_{\text {eff }}$ is identically equal to 1 when $M=1$, i.e., when only one mode is included in $\mathbf{E}$. For $M>1$, the effective degrees of freedom is a measure of how many linearly independent vectors are contained in $\mathbf{E}$. If the modes are orthogonal, then the number of effective degrees of freedom is approximately equal to $M$. As the modes lose orthogonality, $D O F_{\text {eff }}$ decreases. The plot in Figure 2-7 shows the effective degrees of freedom vs. number of modes included in $\mathbf{E}$. The dashed line corresponds to $D O F_{\text {eff }}$ for an ideal filled array that completely spans the water column. The solid line represents the degrees of freedom for the simulation array. Based on the figure, the array samples at least the first 11 modes adequately, but begins to lose degrees of freedom when 12 or more modes are included. Note that $D O F_{\text {eff }}$ is a useful measure of orthogonality for the lowest order modes. Due to aliasing, if only a subset of higher order modes are included in $\mathbf{E}$, then the degrees of freedom measurement might be misleading.

The location of the array and the spacing of the sensors determines the orthogonality of the sampled modeshapes. The number of modes which can be accurately estimated by an array is limited by the orthogonality, but is also influenced by the estimation method. Determining the number of modes to estimate is usually an ad hoc procedure. Analysis of estimation algorithms must address this issue. 


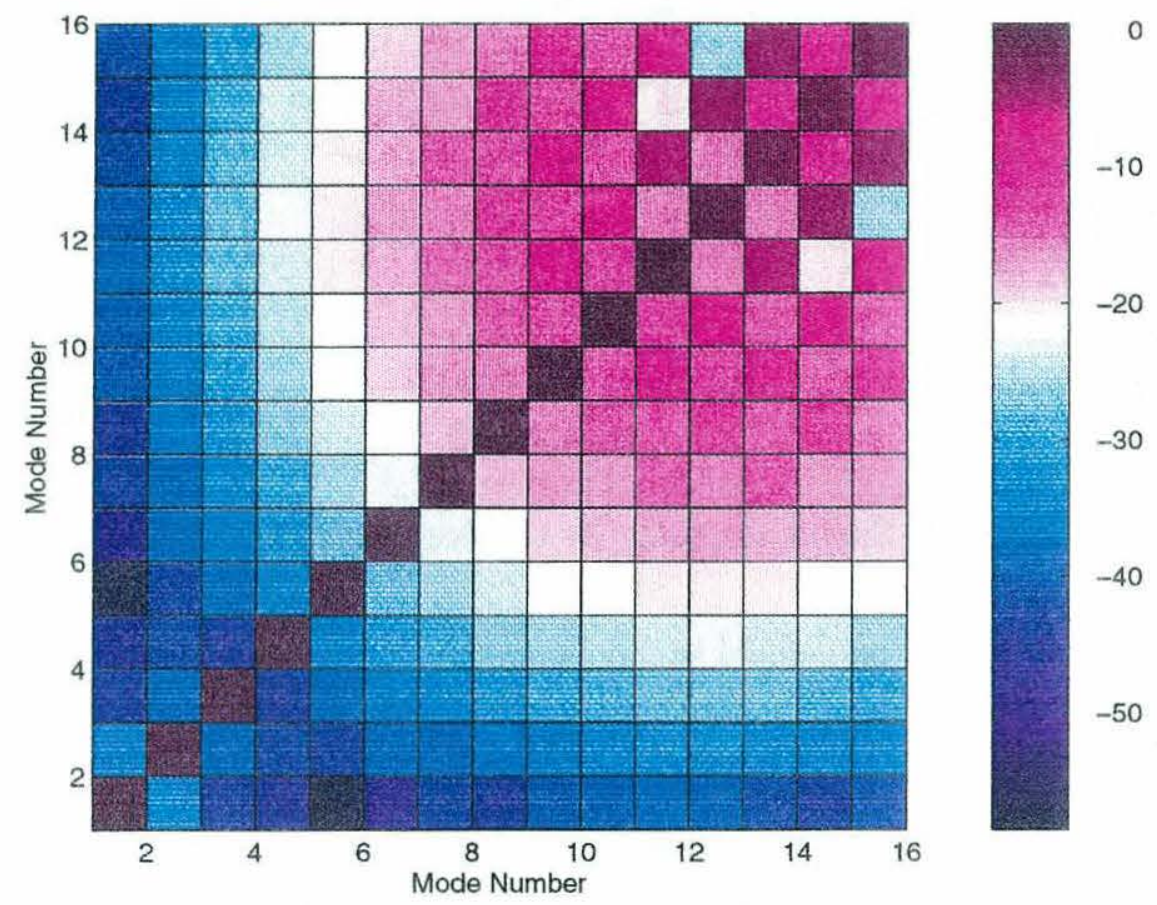

Figure 2-6: Modeshape correlation for the deep water waveguide

\subsubsection{Estimation of the Covariance Matrix}

Many mode estimation algorithms require the sensor covariance matrix $\mathbf{S}$ which contains the second order statistics of the sound field. In practice this matrix must be estimated from the vector time series of pressure measurements taken by the array. The sample covariance is defined as the average of outer products of the data snapshots,

$$
\hat{\mathrm{S}}=\frac{1}{L} \sum_{i=1}^{L} \mathbf{p}_{i} \mathbf{p}_{i}^{\dagger} .
$$

If the field is zero-mean, then $\hat{\mathbf{S}}$ is an unbiased estimate of the true covariance. In addition. if the field is Gaussian (e.g., the model defined in Section 2.3), then Equation 2.28 generates a maximum likelihood estimate of the covariance [19]. The statistics of the sensor covariance estimate are important, especially for adaptive array processing. Goodman has shown that the sample covariance follows a complex Wishart distribution of order $N$ with $L$ degrees of freedom, where $N$ is the number of sensors and $L$ is the number of data snapshots [20]. Many multivariate statistics textbooks, such as the one by Muirhead [21], discuss the Wishart distribution and its relation to the sample covariance. Steinhardt [19] provides an excellent review of the subject with a focus on array processing applications. The properties of 


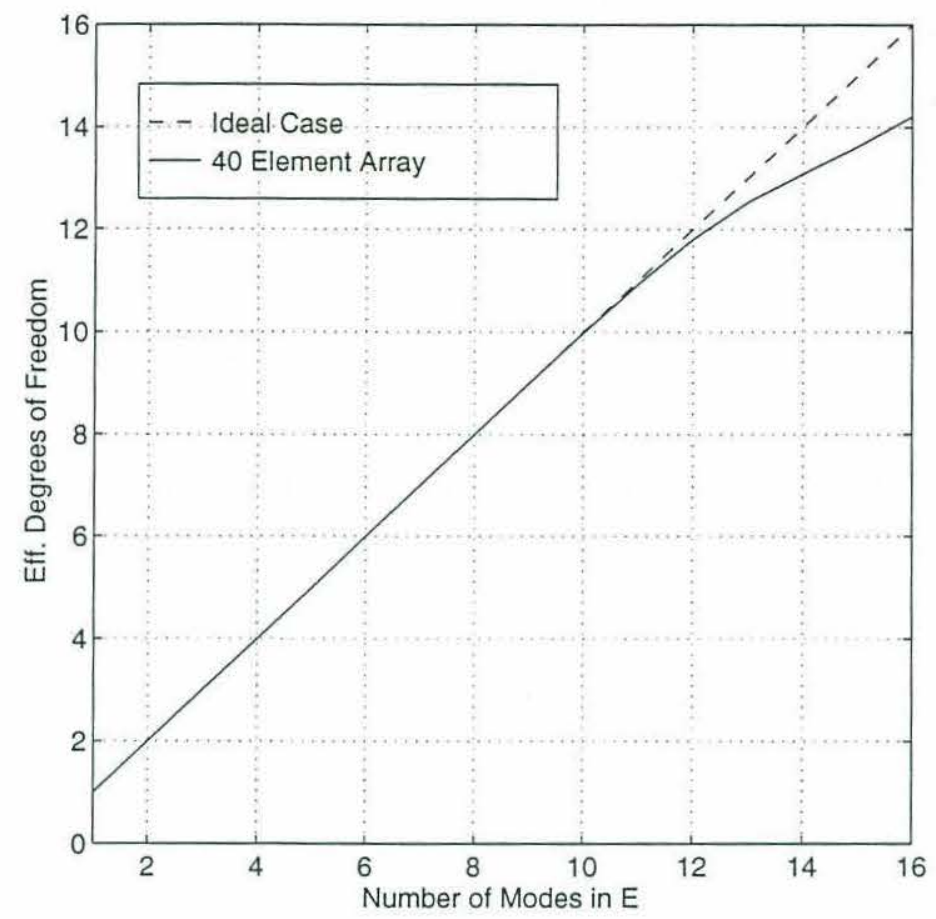

Figure 2-7: Degrees of freedom for the simulation array

the complex Wishart distribution can be used to derive analytical results concerning the statistics of estimates. For example, Capon and Goodman have demonstrated that using a finite number of snapshots in the average leads to bias in algorithms which require the inverse of the sample covariance matrix [22]. Many adaptive algorithms are affected by the number of snapshots used to estimate the covariance. The analysis in the later chapters evaluates the performance of estimators with respect to the number of data snapshots available.

\subsubsection{Signal Coherence}

Signal coherence is another major issue to consider. As discussed in Section 2.3, coherent modes lead to a rank one signal covariance matrix $\mathbf{S}_{\mathrm{S}}$. As coherence is lost, the rank of $\mathbf{S}_{\mathrm{S}}$ increases. Many adaptive algorithms, e.g., those used in matched field processing, exploit the coherence of signals in order to accurately estimate the desired parameters. Since mode coherence is not guaranteed at long ranges, it is important to analyze the effects of coherence on modal estimation strategies. 


\subsubsection{Performance Measures}

This section defines several empirical measures of performance which are useful in analyzing the issues presented in the preceding sections. Since the thesis is primarily concerned with estimating the average powers in the modes, an error vector is defined as follows

$$
\mathrm{e}=\operatorname{diag}\left\{\left|\widehat{\mathrm{S}}_{\mathrm{M}}-\mathrm{S}_{\mathrm{M}}\right|\right\}
$$

A hat distinguishes the estimated mode correlation from its true value. The diag operator indicates that the vector consists of the diagonal elements of the error matrix.

Mode estimates are random variables, thus the error statistics are useful performance measures. The expected error or bias indicates how close the estimates are to the actual parameters on average. Error variance provides a measure of the spread of an estimate around its mean. Note that the error variance is equal to the variance of the estimate. Low variance is a desirable characteristic for an estimator. The Cramer-Rao bound provides a useful lower bound on the variance of an unbiased estimate [23]. Derivation of this limit requires only a knowledge of the conditional probability density of the observations given the true parameters. The bound on the variance of the average power estimates is expressed in terms of the elements of the Fisher information matrix $\mathrm{J}$, e.g., for the $i$ th mode power estimate

$$
\operatorname{var}\left\{\left[\hat{\mathbf{S}}_{\mathrm{M}}\right]_{i i}\right\} \geq\left[\mathrm{J}^{-1}\right]_{i i}
$$

The Fisher information matrix for the mode power estimation problem is derived in Appendix A. Therrien notes that a correction to the bound is available for the case of a biased estimator [23]. The correction term involves the gradient of the bias with respect to the desired parameter vector. Thus, the correction is unnecessary if the bias is due to noise which is independent of the modal signal.

Sometimes a scalar measure of the error is useful. The total error is defined as the trace of the error matrix, i.e.,

$$
\text { Total Error }=\mathrm{TR}\left\{\left|\widehat{\mathrm{S}}_{\mathrm{M}}-\mathrm{S}_{\mathrm{M}}\right|\right\}
$$




\subsection{Summary}

This chapter has presented a general mathematical model for mode propagation in an ocean waveguide. Both deterministic and random channels fit into the same framework, provided that the propagation parameters are specified appropriately. Section 2.3 has defined the acoustic mode estimation problem in an array processing context. The final section has outlined important issues which will be used in the performance analysis of the estimators developed in the following chapters. In addition this chapter has described a deep water environment and an array geometry for numerical simulations. 


\section{Chapter 3}

\section{Least Squares Methods}

The previous two chapters have motivated and defined the modal estimation problem. In particular, Chapter 2 developed the necessary background and stressed the importance of different aspects of the signal processing. The purpose of this chapter is to review standard methods for estimating the modal powers.

In theory the orthogonality of the normal modes permits the use of spatial matched filters. For example, Ferris [24] determines the weight associated with each mode by "matching" the received signal to the sampled modeshape. This method relies on the orthogonality of the sampled modeshapes, which is not always a valid assumption in practice. Recall from Chapter 2 that the orthogonality of the modes depends on the sensor spacing and position of the array. Since logistics and funding often limit the number of sensors that can be deployed, the desired modes are not always orthogonal. If the sampled modeshapes are not orthogonal, power from one mode can leak into estimates of the adjacent modes. Conventional approaches often apply Least Squares (LS) estimation theory to reduce the effects of non-orthogonal modeshapes. LS techniques compute a set of weights which minimize the total squared error between the measured and estimated modal fields.

Many researchers have addressed the problem of estimating the modes in an acoustic signal. Clay was the first to recognize the important link between the modal decomposition and array processing [25]. Hinich developed a maximum likelihood method for depth localization using the normal modes [26]. Since that time, a class of matched field processing algorithms based on normal mode theory has been developed. A survey of the current literature indicates that Matched Mode Processing (MMP) applications generate most of the 
mode estimation research $[2,3,4,27]$. All of the standard estimation techniques used for MMP are based on minimizing a squared error criterion. A review article by Voronovich et. al. compares five modal estimators using numerical simulations and experimental data [8]. Two recent theses have addressed the modal estimation problem. Polcari provides a thorough overview of conventional modal beamforming, including a practical application to a coherent Arctic channel [9]. Sperry discusses the use of least squares modal filtering in the context of a long-range propagation experiment [28].

This chapter is organized as follows. Section 3.1 develops the standard least squares estimator and analyzes it using a few numerical examples. The performance evaluation addresses the main issues outlined in Chapter 2: ambient noise, mode orthogonality, estimated covariances, and signal coherence. Finally, Section 3.2 summarizes the characteristics of the LS estimator, thereby motivating the search for a new approach.

\subsection{Standard Least Squares}

Standard modal estimation algorithms produce time series using a least squares approach to generate the mode amplitudes for each data snaphsot. For a single pressure vector $\mathrm{p}$, the LS technique minimizes a squared error criterion in order to estimate the mode amplitudes, i.e.,

$$
\widehat{\mathrm{a}}_{\mathrm{ls}}=\min _{\mathrm{a}}|\mathrm{p}-\mathrm{Ea}|^{2} .
$$

This method implicitly assumes that the received pressure consists only of the modal signal Ea and ignores the noise components. The minimization problem stated above has a well-known solution [29, 30, 31],

$$
\widehat{a}_{l s}=\left(E^{\dagger} E\right)^{-1} E^{\dagger} p
$$

Consider the case where the received pressure consists of the modal signal only. It is trivial to show that $\widehat{a}_{1 \mathrm{~s}}$ is equal to the true mode amplitudes, provided that $\left(\mathbf{E}^{\dagger} \mathrm{E}\right)^{-1}$ exists and the number of modes in the estimate is greater than the number of modes in the signal. Now suppose that additive noise corrupts the pressure measurements. The linearity of the

LS processor implies that the noise corrupts the resulting mode estimates as well. For least squares methods to be effective, the SNR must be high enough so that the signal 
components dominate the noise.

Note that if the modes are orthogonal then $\left(\mathbf{E}^{\dagger} \mathbf{E}\right)^{-1}$ is a multiple of the identity matrix, and the least squares solution corresponds to Ferris' matched filtering approach. If the modes are not orthogonal, then the inverse in Equation 3.2 is usually computed using the Singular Value Decomposition (SVD).

Recall that the desired parameters to estimate are the average powers in the modes, i.e., the diagonal terms of the modal correlation matrix. Polcari [9] suggests that the second order modal statistics, can be obtained from the time series as an average of outer products

$$
\hat{\mathrm{S}}_{\mathrm{M} \_ \text {LS }}=\frac{1}{L} \sum_{i=1}^{L} \mathrm{a}_{\mathrm{ls}_{i}} \mathrm{a}_{\mathrm{ls}_{i}}^{\dagger}
$$

where $L$ is the number of data snapshots available. Since the least squares processor is linear, Equation 3.3 is obriously equivalent to

$$
\hat{\mathrm{S}}_{\mathrm{M} \_ \text {LS }}=\left(\mathrm{E}^{\dagger} \mathrm{E}\right)^{-1} \mathrm{E}^{\dagger}\left\{\frac{1}{L} \sum_{i=1}^{L} \mathrm{p}_{i} \mathrm{p}_{i}^{\dagger}\right\} \mathrm{E}\left(\mathrm{E}^{\dagger} \mathrm{E}\right)^{-1}=\left(\mathrm{E}^{\dagger} \mathrm{E}\right)^{-1} \mathrm{E}^{\dagger} \hat{\mathrm{S}} \mathrm{E}\left(\mathrm{E}^{\dagger} \mathrm{E}\right)^{-1}
$$

where $\hat{\mathrm{S}}$ is the estimated sensor covariance matrix.

The following example illustrates the basic characteristics of the least squares estimator. Subsequent sections examine specific aspects of the estimator's performance in more detail.

\section{Deep Water Example}

The simulation environment is the deep water waveguide described in Chapter 2. Figure 24 shows the modeshapes for the $70 \mathrm{~Hz}$ narrowband source used in the following examples. It is convenient to define a standard test signal for use throughout the thesis. The signal consists of a coherent sum of the lowest 8 modes: the odd modes $(1,3,5,7)$ are excited at a reference level of $0 \mathrm{~dB}$ and the even modes $(2,4,6,8)$ are excited at $-10 \mathrm{~dB}$. Modes 9 and higher are not present in the signal. ${ }^{1}$ Additive noise in the environment consists of white sensor noise only. The power level of the white noise controls the effective signal-to-noise ratio for each example. As defined in Section 2.4.1, the input SNR is the geometric mean of the SNR's at each sensor in the array. The 40-element simulation array, described in

\footnotetext{
${ }^{1}$ Parameters of the test signal are somewhat arbitrary. The alternating pattern in the first 8 modes is for visualization purposes. The absence of modes 9 and higher is useful in determining how well the processing handles nulls in the modal power spectrum.
} 
Section 2.4.2, samples the water column. Least squares processing attempts to resolve the power in the first 10 modes of the waveguide.

First consider the case where the sensor covariance is known exactly and does not have to be estimated from snapshot data. While this is clearly not a practical assumption, the ideal covariance case indicates the best possible performance of the estimator. Figure 3-1 shows the ideal LS estimates of the power in the first 10 modes for three different signalto-noise ratios: $10 \mathrm{~dB}, 0 \mathrm{~dB}$, and $-10 \mathrm{~dB}$. Circles denote the true power distribution and
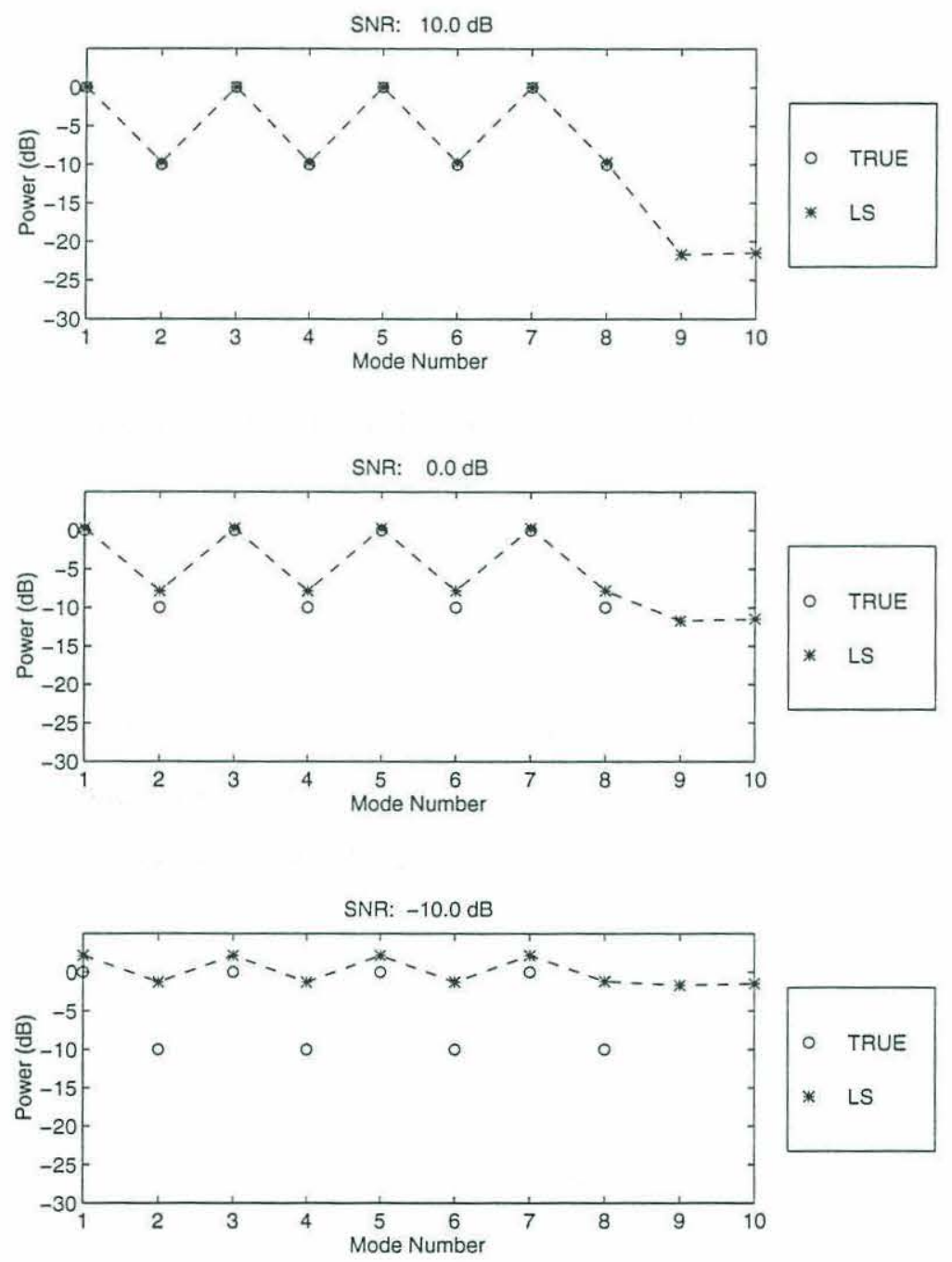

Figure 3-1: Least squares power estimates for the deep water example

asterisks represent the LS mode estimates. The dashed line which interpolates the LS results is for viewing purposes; the estimate only exists for integer mode numbers. The top plot 
shows the estimates for the $10 \mathrm{~dB}$ SNR case. The alternating pattern of the distribution for modes 1-8 is clear, and the estimates of the null modes (9 and 10) are more than $20 \mathrm{~dB}$ down from the reference level. In the $0 \mathrm{~dB}$ SNR case (middle plot) the LS estimates for modes 1-8 are biased, with errors more pronounced in the lower power modes. Ideally the estimator should show nulls in the spectrum for modes 9 and 10, but at $0 \mathrm{~dB}$ SNR the LS estimate of these modes is on the order of $-10 \mathrm{~dB}$ (the level of the even modes). Bias errors are the most severe in the $-10 \mathrm{~dB}$ SNR case where the LS processor indicates that modes 9 and 10 are almost as energetic as the rest of the modes.

In order to understand the behavior shown in Figure 3-1, consider analytical results for the ideal least squares estimate. For a model including a signal plus white sensor noise, the sensor covariance $\mathbf{S}$ is given below

$$
\mathrm{S}=\mathrm{E}^{\dagger} \mathrm{S}_{\mathrm{M}} \mathrm{E}+\sigma_{w}^{2} \mathrm{I}
$$

Assuming that the number of modes in the estimate is greater than or equal to the number of modes in the signal, the ideal least squares estimate, becomes ${ }^{2}$

$$
\mathbf{S}_{\mathrm{M} \_L S}=\mathbf{S}_{\mathrm{M}}+\sigma_{w}^{2}\left(\mathbf{E}^{\dagger} \mathbf{E}\right)^{-1}
$$

According to Eq. 3.6, the power in each mode is biased by a constant term which depends on the white noise level and the inverse of the modeshape correlation matrix. If the modes are orthogonal and scaled equally, then $\left(\mathrm{E}^{\dagger} \mathbf{E}\right)^{-1}$ is a multiple of the identity matrix and all modes are biased by the same amount. As orthogonality is lost due to inadequate sampling, the processor may bias some modes more than others.

Figure 3-2 shows the error in the LS power estimates for the deep water example. Recall from Equation 2.29 that the error vector e is defined as the absolute value of the differences in the true powers and the estimated powers. The three plots show the errors on a log scale $\left(10 \log _{10} \mathbf{e}_{i}\right)$ for the SNR levels used in the deep water example. As expected, errors increase as SNR decreases. The plots reveal that the bias is roughly constant across the modes. This is reasonable since the array adequately samples the first 10 modes (see the

\footnotetext{
${ }^{2}$ Notation: In the rest of the thesis a hat is used to distinguish sample covariance results from ideal covariance results, e.g., $\mathrm{S}_{\mathrm{M}_{-} \text {LS }}$ represents the ideal LS estimate of the mode correlation matrix and $\widehat{\mathbf{S}}_{\mathrm{M} \_L S}$ is the corresponding sample covariance estimate.
} 

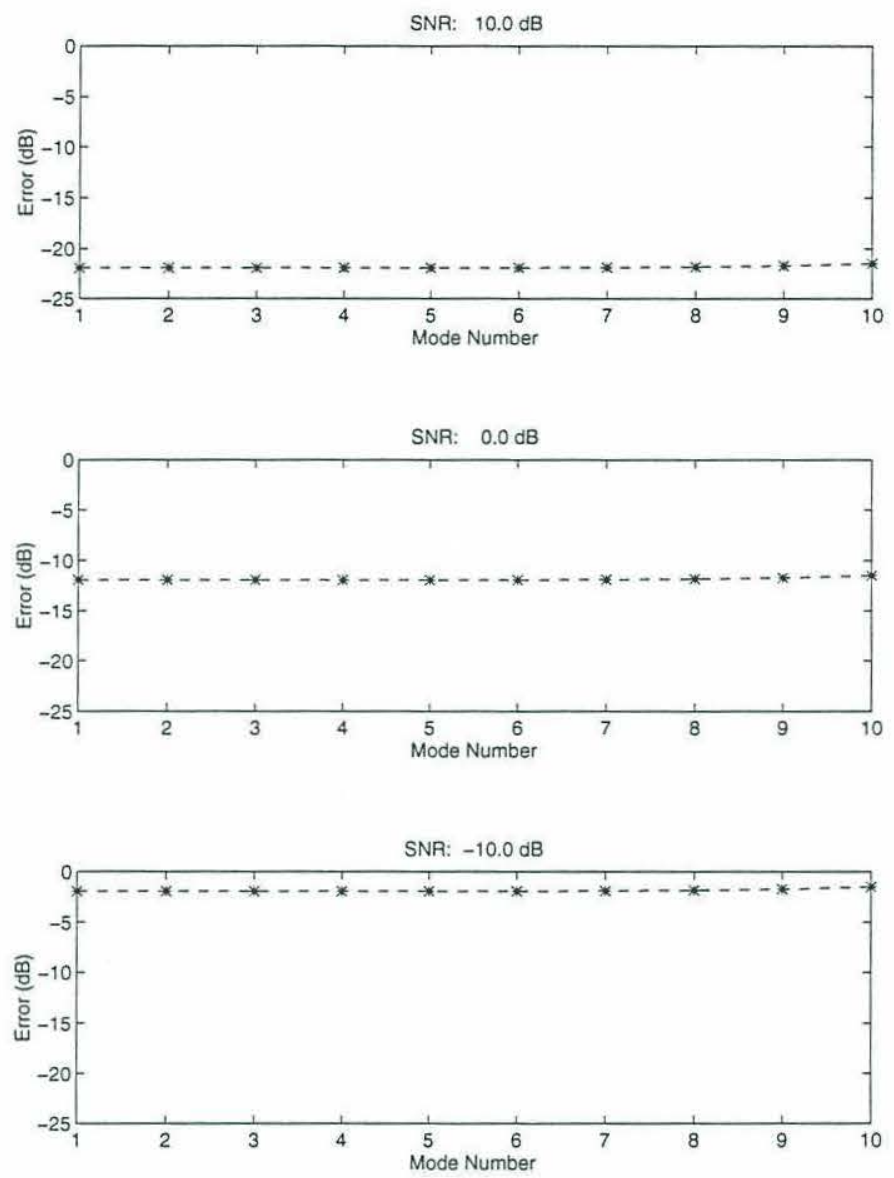

Figure 3-2: Error in least squares power estimates for the deep water example

discussion in Section 2.4.2).

Several factors influence the error in the least squares estimate. The purpose of the following sections is to consider each of these factors separately. The next section describes the error as a function of the noise. Section 3.1.2 focuses on mode orthogonality and determination of the number of modes to estimate. Next, Section 3.1.3 examines the practical issues associated with estimating the sensor covariance matrix from the data. Finally, Section 3.1.4 addresses the issue of signal coherence.

\subsubsection{Ambient Noise}

An important aspect of the performance evaluation is to examine the effects of noise on the modal power estimates. Least squares techniques minimize the squared error without regard to the ambient noise conditions. As a result, the LS processor relies on the powers associated with the modal signal being larger than the noise components in order to generate 
accurate estimates.

Based on Equation 3.6, the error in mode $j$ is proportional to the white noise power, i.e.,

$$
e_{j}=\sigma_{w}^{2}\left[\left(\mathbf{E}^{\dagger} \mathbf{E}\right)^{-1}\right]_{j j} .
$$

According to Equation 3.7, the error is a linear function of the noise power and is independent of the true power in the mode. Consider an example. The parameters are identical to the deep water scenario described above with the exception that the white noise power is adjusted so that the SNR varies from $-20 \mathrm{~dB}$ to $20 \mathrm{~dB} .{ }^{3}$ Figure $3-3$ shows the log of the error versus SNR for modes 1, 2, and 10. The plots indicate that the error is a linear function of the SNR which verifies that the error is also linear function of the noise power. Note
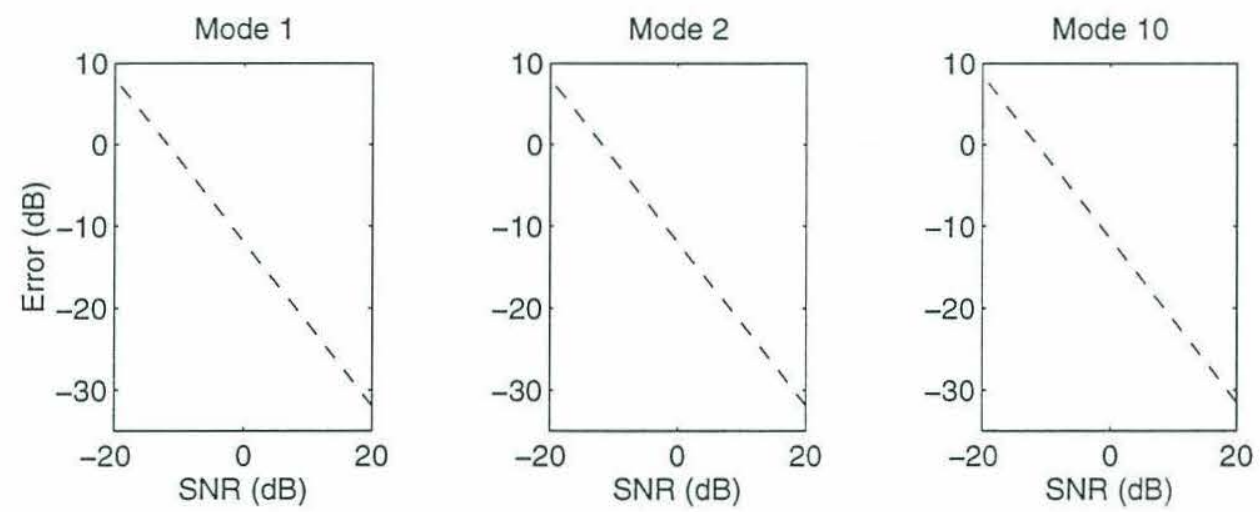

Figure 3-3: Error vs. SNR for modes 1, 2, and 10 of the deep water waveguide. Ambient noise consists of white sensor noise only.

that the errors for all three modes appear to be identical. This is expected since the array adequately samples 10 modes, therefore $\left[\left(\mathbf{E}^{\dagger} \mathbf{E}\right)^{-1}\right]_{j j}$ is approximately constant for all $j$. The effect of non-orthogonal sampled modeshapes is discussed in the next section.

Consider the case where a discrete noise source with the same narrowband frequency as the signal is added to the environment. The noise source is uncorrelated with the signal and has a modal correlation matrix, $\mathrm{S}_{\mathrm{M}_{-} \mathrm{D}}$. In this case the LS estimate of the correlation becomes

$$
\mathrm{S}_{\mathrm{M} \_ \text {LS }}=\mathrm{S}_{\mathrm{M}}+\mathrm{S}_{\mathrm{M} \_\mathrm{D}}+\sigma_{w}^{2}\left(\mathbf{E}^{\dagger} \mathbf{E}\right)^{-1}
$$

\footnotetext{
${ }^{3} \mathrm{SNR}$ is defined as in Section 2.4.1.
} 
leading to the following expression for the error in mode $j$

$$
e_{j}=\left[\mathbf{S}_{\mathrm{M}_{-} \mathrm{D}}\right]_{j j}+\sigma_{w}^{2}\left[\left(\mathbf{E}^{\dagger} \mathbf{E}\right)^{-1}\right]_{j j} .
$$

A powerful discrete source can overwhelm the true signal, resulting in large errors. The least squares processor is clearly incapable of resolving multiple discrete sources.

\subsubsection{Modal Orthogonality}

For a fixed noise level, the main issue to consider is the number of modes which can be reliably estimated using the least squares technique. Based on the discussion in Section 2.4.2, the orthogonality of the sampled modeshapes directly affects the accuracy of the estimate. The $\left[\left(\mathrm{E}^{\dagger} \mathrm{E}\right)^{-1}\right]_{j j}$ term in Equation 3.7 contains the effects of modal orthogonality on the LS error. If the modes are perfectly orthogonal, then $\mathrm{E}^{\dagger} \mathrm{E}$ is a multiple of the identity matrix and the resulting error is constant. If the sampled modeshapes are not orthogonal, then $\mathrm{E}^{\dagger} \mathrm{E}$ is not a multiple of the identity and the error is a function of the mode number $j$. As

more non-orthogonal modes are included in the estimate, $\mathrm{E}^{\dagger} \mathrm{E}$ approaches rank deficiency and the errors increase. In the limit as $\mathrm{E}^{\dagger} \mathrm{E}$ becomes singular (i.e., has a zero eigenvalue), the error becomes unbounded. Recall that the effective degrees of freedom $\left(D O F_{\text {eff }}\right)$ is often a useful measure of the orthogonality of the sampled modeshapes.

As an extreme example, consider using the simulation array to estimate the power levels in the lowest 16 modes of the deep water waveguide. Figure 2-7 indicates that there are only 14 effective degrees of freedom for the 16-mode case; clearly the sampled modeshapes are not orthogonal. Assume that the input signal consists of an alternating pattern of the first 16 modes: the odd modes are excited at $0 \mathrm{~dB}$ and the even modes are excited at $-10 \mathrm{~dB}$. The white noise level is fixed so that the average SNR is $0 \mathrm{~dB}$. Ideal covariance matrices are used in the simulation. Figure $3-4$ shows the resulting error vector $\left(10 \log _{10} \mathbf{e}_{j}\right)$ for the 16-mode estimate. The error curve is constant at around $-10 \mathrm{~dB}$ for the low order modes, but increases dramatically above mode 9 . This is due to the fact that the higher order modes are not adequately sampled by the array. Based on this simulation the errors in the higher order modes are significant when $\mathrm{E}^{\dagger} \mathrm{E}$ has lost 2 degrees of freedom. The next example examines how the error varies as a function of the number of modes included in the estimate. 


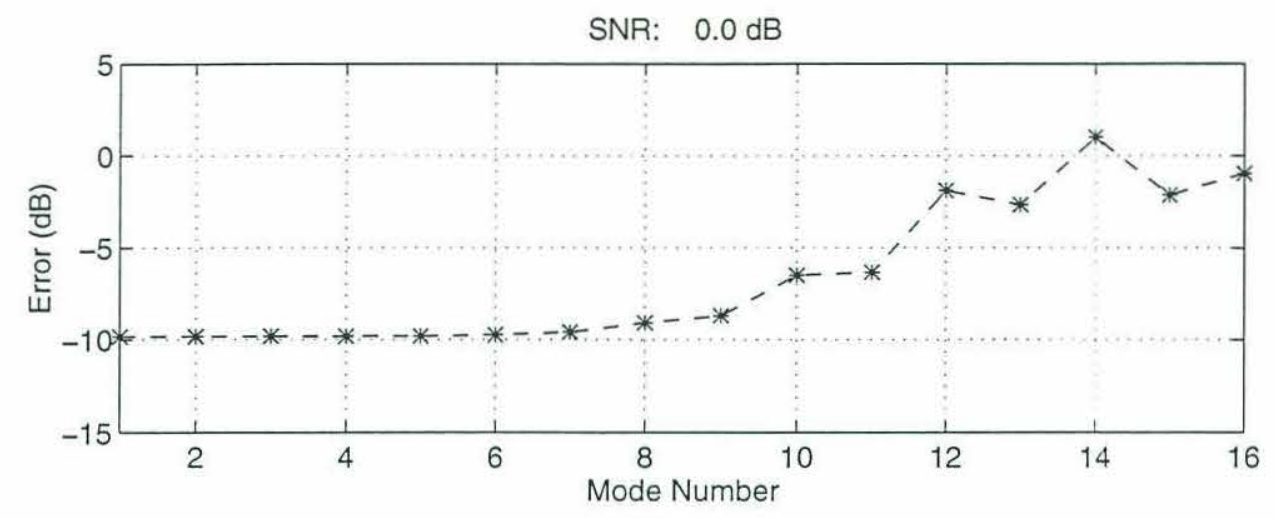

Figure 3-4: Error vector for the 16-mode example using the deep water waveguide

The parameters of this example are identical to the first deep water example used in this chapter, i.e., the test signal consists of the lowest 8 modes in an alternating $0 /-10 \mathrm{~dB}$ pattern. The number of modes to estimate is varied from 8 (the number of modes in the signal) up to 16. A useful scalar measure of performance is the total error defined in Chapter 2, e.g. for the LS estimator,

$$
\text { Total Error }=\mathrm{TR}\left\{\left|\widehat{\mathrm{S}}_{\mathrm{M} \_L S}-\mathrm{S}_{\mathrm{M}}\right|\right\}
$$

If the modes are orthogonal and scaled equally, then the modeshape correlation matrix is a multiple of the identity matrix, i.e., $\mathbf{E}^{\dagger} \mathbf{E}=\beta \mathbf{I}$. Under these assumptions the total error (using equations 3.6 and 3.10 ) is shown below

$$
\text { Total Error } \text { orth }=M \cdot \frac{\sigma_{w}^{2}}{\beta}
$$

where $M$ is the number of modes estimated. In other words given a set of orthogonal sampled modeshapes, the total error is a linear function of $M .{ }^{4}$ In the case of non-orthogonal modes, the total error should exceed the amount in Eq. 3.11. Figure 3-5 verifies this assertion. The plot shows the total error vs. number of modes to estimate. The solid line is the total error associated with a set of perfectly orthogonal modes. The asterisks represent the actual total error (using ideal covariances) for the modes of the deep water waveguide which are sampled using the 40-element simulation array.

\footnotetext{
${ }^{4}$ This argument implicitly assumes that $M$ is greater than or equal to the number of modes actually present in the signal.
} 
SNR: $\quad 0.0 \mathrm{~dB}$

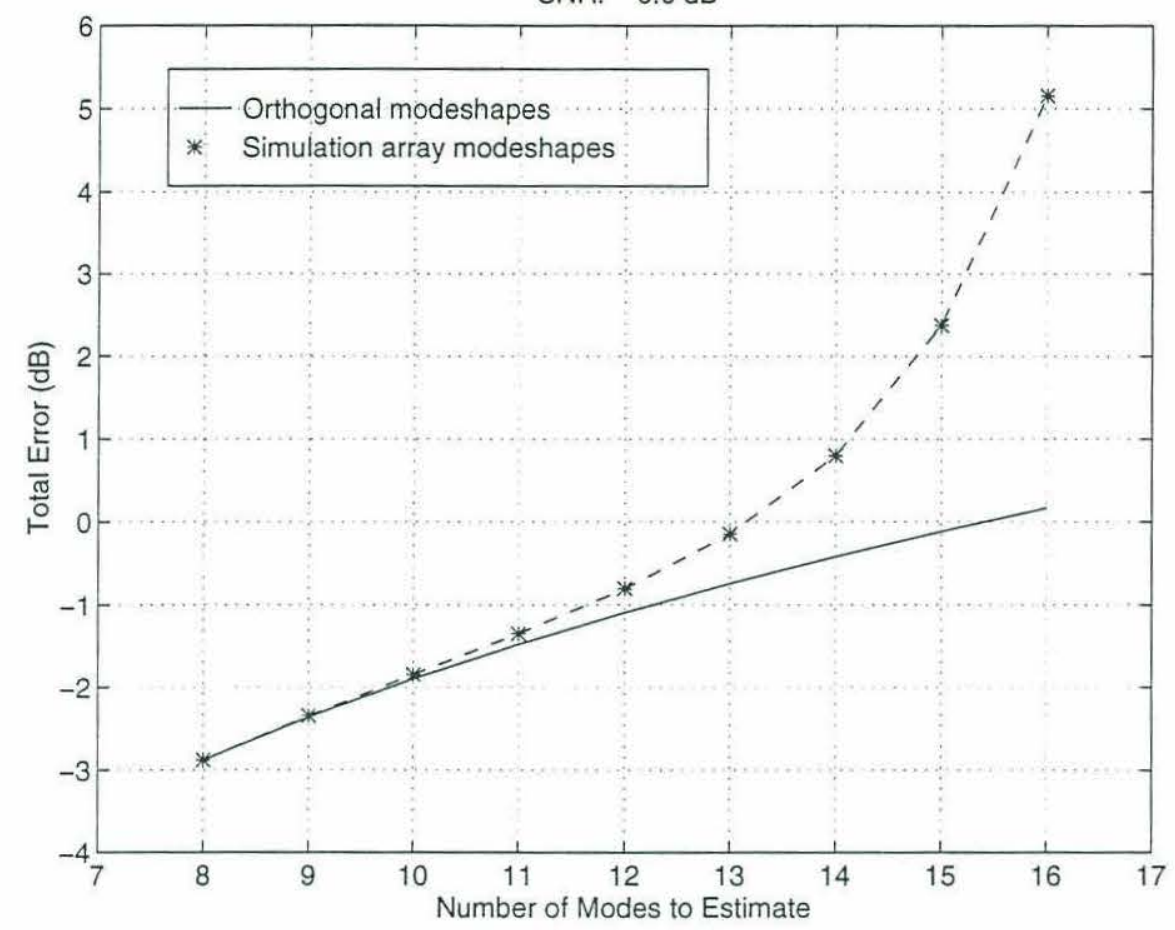

Figure 3-5: Total error vs. number of modes to estimate for the deep water example

A comparison of Figures 2-7 and 3-5 reveals that $D O F_{\text {eff }}$ is a useful measure of the number of lower order modes that can be accurately estimated using a particular array configuration. As the effective degrees of freedom deviate from the ideal, the total error in the estimate grows. Note that the errors increase rapidly once a whole degree of freedom is lost. The solution to this problem is to include only an approximately orthogonal subset of modes in the estimate. Of course, if the higher order modes (which are not being estimated) are present in the signal, the power from those modes biases the results.

Consider the difficulty of estimating a set of modes which is larger than the subset adequately sampled by the array. There are several ways to address this problem. One method is to use diagonal loading to stabilize the matrix inverse. The geophysical literature refers to this general approach as damped least squares. See the texts by Menke [30] or Aki and Richards [31] for more details. Sperry discusses the use of damped least squares in the specific context of an acoustic mode estimation problem [28]. A second approach to the problem is to use the subspace reduction method suggested by Yang [4]. The comparative study by Voronovich et. al. [8] includes both of these methods. 


\subsubsection{Estimated Covariance Issues}

The preceding examples have used an ideal sensor covariance to compute the estimates, but in practice the LS processor uses the sample covariance matrix, $\widehat{\mathbf{S}}$. As a result the modal power estimates are random variables that can be characterized by their second order statistics. Appendix B derives the mean and variance of the least squares power estimates using known characteristics of the sample covariance matrix. Results are summarized below.

Assuming zero-mean data, $\widehat{\mathbf{S}}$ is an unbiased estimate of $\mathbf{S}$, therefore the expected value of the LS estimate is equal to the ideal covariance result. For the case of only white noise, the expected power in mode $j$ can be written

$$
\mathcal{E}\left\{\widehat{P}_{j \_\mathrm{LS}}\right\} \equiv \bar{P}_{j \_\mathrm{LS}}=P_{j}+\sigma_{w}^{2}\left[\left(\mathbf{E}^{\dagger} \mathbf{E}\right)^{-1}\right]_{j j}
$$

where $P_{j}$ is the true power in the mode. Note that the least squares bias depends only on the white noise level and is unaffected by the number of data snapshots available.

It can be shown that the power estimate for each mode is a scaled chi-squared random variable with $L$ degrees of freedom, where $L$ is the number of snaphsots used to form the sample covariance matrix. Using this fact, the variance for the power estimate for mode $j$ becomes

$$
\operatorname{Var}\left\{\hat{P}_{j \perp S}\right\}=\frac{\left(\bar{P}_{j L S}\right)^{2}}{L} .
$$

The variance is inversely proportional to the number of snapshots. According to Section 2.4.5 and Appendix A, the Cramer Rao bound has the same $1 / L$ dependence.

Based on Equations 3.12 and 3.13, it is possible to predict bias and variance for the least squares estimates. The following example contrasts the predictions with the results of a Monte Carlo simulation. The parameters of the simulation are identical to the deep water example using the test signal. Each data point is the result of averaging over 1000 independent trials. Figure 3-6 shows the error vs. the number of snapshots for mode 1. Predicted error is shown along with the Monte Carlo results. The error is constant (associated with the white noise bias) as predicted by Eq. 3.12. Figure 3-7 shows the estimator variance vs. number of snapshots. The predicted and Monte Carlo values are shown, along with the Cramer Rao bound (calculated in Appendix A). Both the predicted and Monte Carlo values basically agree with the Cramer Rao bound, meaning that the estimate is approximately 


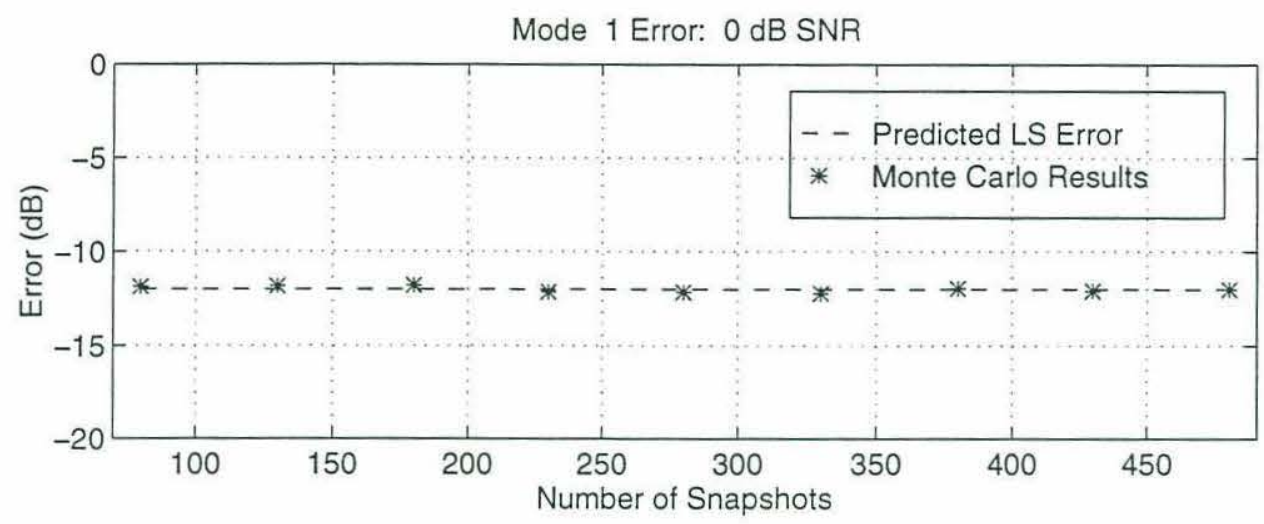

Figure 3-6: Bias error vs. number of snapshots for mode 1. Predicted and Monte Carlo results for the LS estimator are shown.

efficient.

\subsubsection{Signal Coherence}

A useful property of the least squares formulation is that it does not depend on the correlation structure of the incident signal. For any $\mathbf{S}_{\mathrm{M}}$, the expected value of $\widehat{\mathbf{S}}_{\mathrm{M} \_L S}$ is always equal to the true correlation matrix plus a noise term. Thus, the error remains constant as a function of coherence, provided that all other signal parameters are fixed. Since channel characteristics are not always available a priori, this is clearly an advantage of the least squares processor.

\subsection{Summary}

This chapter has reviewed conventional mode estimation algorithms, in particular the standard least squares processor. The primary drawback of the LS estimator is that it relies on the acoustic signal being much louder than the ambient noise. This is not always the case in practice, especially for long-range experiments. In addition the orthogonality of the sampled modeshapes significantly affects the accuracy of the estimates. It was shown that the effective degrees of freedom in the modeshape correlation matrix is a useful measure of orthogonality. The LS estimator does have two advantages: it is relatively easy to implement and a priori knowledge of the coherence structure is not necessary. 


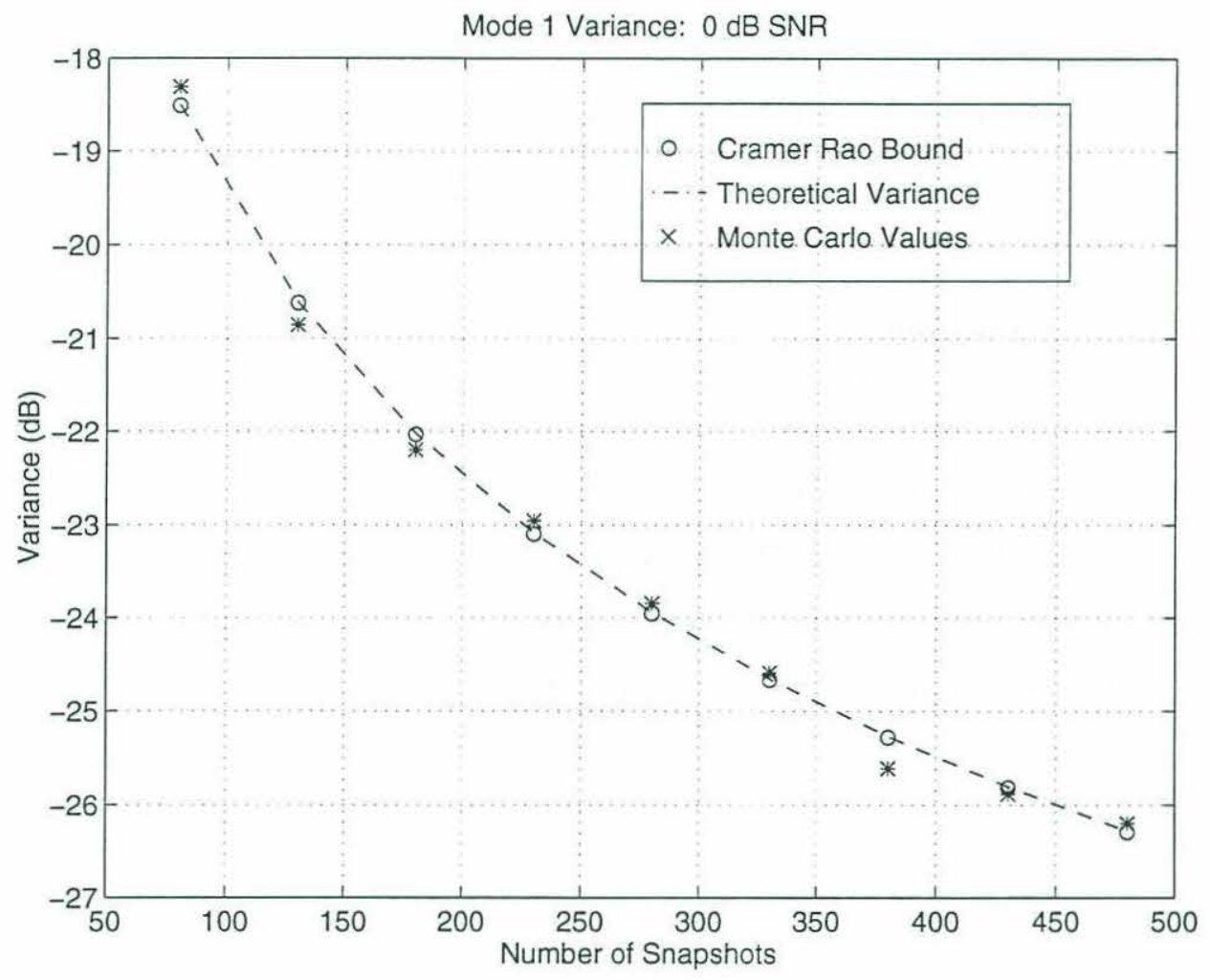

Figure 3-7: Variance vs. number of snapshots for mode 1. Predicted and Monte Carlo results for the LS estimator are shown along with the Cramer Rao bound. 


\section{Chapter 4}

\section{Adaptive Estimation of Coherent Modes}

As demonstrated in the previous chapter, the orthogonality of the sampled mode shapes and the signal-to-noise ratio strongly influence the performance of modal estimators. The methods discussed in Chapter 3 are based on minimizing a squared error criterion. Least squares methods perform poorly when the sampled modes are not orthogonal and the SNR is low. In this chapter an alternative modal estimation technique is introduced which maximizes received signal power rather than minimizing a squared error criterion. The following discussion focuses on the coherent mode case. Chapter 5 presents an extension of these techniques to a more general family of signals.

The purpose of this chapter is to develop methods for estimating the power distribution in a spatial signal consisting of a coherent sum of modes. Power spectral estimation is a well-known problem which is addressed in numerous reference texts [32, 33, 23, 34]. In particular, Johnson and Dudgeon provide a good introduction to the estimation of spatial power spectra using arrays [34]. There are three basic approaches to this array processing problem. Conventional methods apply deterministic weightings to the data vectors to form spectral estimates. These deterministic weight vectors do not change to account for different noise backgrounds. A second category of spectral estimators is data-adaptive because the algorithms use weight vectors which are derived from the statistics of the incident field. An example of this type of algorithm is the Minimum Variance (MV) method of Capon [35]. Eigenanalysis methods such as MUltiple SIgnal Classification (MUSIC) [36] and Estimation 
of Signal Parameters Via Rotational Invariance Techniques (ESPRIT) [37] represent the third type of estimator. These techniques rely on separating the data into signal and noise subspaces. They are also adaptive in the sense that they use information from a model of the field to reduce the effects of noise on the estimate.

The methods listed above have one thing in common. They all assume that the spatial structure of the received signal is known, e.g., a planewave. For this research the planewave assumption is not valid. Instead, the received signal consists of a weighted coherent sum of modes. The weights indicate the relative distribution of power among the normal modes. According to Section 2.3, the coherent mode estimation problem involves determining parameter estimates for the signal power, $\mathcal{P}_{\mathrm{T}}$, and the relative modal distribution, $\mathrm{a}_{\mathrm{T}}$. One reasonable method for determining an estimate for $\mathrm{a}_{\mathrm{T}}$ is to choose the distribution which maximizes the received power. This is equivalent to doing direction-finding with an array of sensors by locating the peaks in the power spectrum.

Locating the spectral peak involves solving a multidimensional optimization problem. The straightforward method of computing the spectrum for all possible values of the distribution $\mathrm{a}_{\mathrm{T}}$ is impractical, especially when the desired number of modes to estimate is large. Fortunately, an efficient method exists for finding the peaks in certain types of power spectra. A recent paper describes a similar problem for direction-finding with an array of diversely polarized antennas [38]. In this paper Ferrara and Parks compute an angle-ofarrival spectrum by maximizing a spectrum parameterized on angle and polarization over all possible polarizations. They note that the optimization problem reduces to an eigenvalue problem which is relatively easy to solve. A similar method solves the optimization problem for modal estimation.

This chapter develops two modal estimators based on the minimum variance and MUSIC formulations considered by Ferrara and Parks. The following section contains the derivation and analysis for the minimum variance estimator. Section 4.2 presents the MUSIC modal estimator. The performance analysis for each algorithm addresses the four main issues outlined in Chapter 2: ambient noise, mode orthogonality, estimated covariance, and signal coherence. The numerical examples used are consistent with those in Chapter 3 to permit a comparative study of the algorithms. 


\subsection{Minimum Variance Modal Estimator}

The minimum variance processor is the result of a constrained optimization problem. The output of the processor is computed as a weighted linear combination of the received signal at each sensor. Let g represent an ideal signal or "replica" incident on the array. The weight vector $\mathrm{w}$ applied to the vector of sensor outputs $\mathrm{p}$ should emphasize any signal that looks like the replica and suppress all other signals. Minimizing the output power of the processor subject to a unity gain constraint effectively suppresses signals which do not resemble the ideal while passing the ideal signal with unity gain. Thus the minimum variance weight vector is the solution to the following problem

$$
\min _{\mathbf{w}} \mathcal{E}\left\{\left|\mathrm{w}^{\dagger} \mathrm{p}\right|^{2}\right\} \quad \text { subject to } \quad\left\{\mathrm{g}^{\dagger} \mathrm{w}\right\}=1
$$

The weight vector is easily obtained using standard constrained optimization techniques

$$
\mathrm{w}_{\mathrm{mv}}=\frac{\mathrm{S}^{-1} \mathrm{~g}}{\mathrm{~g}^{\dagger} \mathrm{S}^{-1} \mathrm{~g}}
$$

The output power of the minimum variance processor is given below

$$
\mathcal{P}_{\mathrm{mv}}=\frac{1}{\mathrm{~g}^{\dagger} \mathrm{S}^{-1} \mathrm{~g}}
$$

The minimum variance technique is also known in the literature as Capon's method, the Maximum Likelihood Method (MLM), or the Minimum Variance Beamformer (MVBF). Capon introduced the minimum variance estimator in 1969. Since that time it has found wide application in both spectral estimation (for time series data) and array processing. The terminology associated with this estimator is often confusing since the weight vector is not the maximum likelihood solution for any known estimation problem. When the input signal can be characterized as a zero-mean random vector, the average output power minimized above is the variance; hence the name minimum variance. For a rank one signal covariance, Steinhardt [19] notes that the minimum variance weight vector also maximizes the signal-to-noise ratio at the processor output.

Ferrara and Parks have used the minimum variance formulation to estimate an angle-ofarrival power spectrum using an array of diversely-polarized antennas. For their algorithm 
the replica vector is a function of angle-of-arrival and the polarization parameters. They maximize the minimum variance power spectrum over all possible polarizations to obtain the power at each angle of interest. The optimization is done efficiently by formulating it as an eigenvalue problem.

In mode estimation the desired parameters are the relative distribution $\mathrm{a}_{\mathrm{T}}$, which determines the spatial structure of the received signal, and the power $\mathcal{P}_{\mathrm{T}}$ in the received signal. These can be estimated using the Ferrara/Parks method. The relative weights of the modes are determined by choosing the set of weights which maximize the minimum variance power estimate. The estimate of the signal power corresponds to the maximum power. Again, the optimization is done by solving an eigenvalue problem.

The replica vector for the modal signal in the coherent case is

$$
\mathrm{g}=\mathrm{Ea} \text {. }
$$

where $\mathbf{a}$ is defined as a unit-length vector. The resulting minimum variance power spectrum is

$$
\mathcal{P}_{\mathrm{mv}}=\frac{1}{\mathrm{a}^{\dagger} \mathrm{E}^{\dagger} \mathrm{S}^{-1} \mathrm{Ea}}
$$

Using the criterion suggested by Ferrara and Parks, the estimated modal distribution $\hat{a}_{M V}$ is chosen as the vector a which maximizes $\mathcal{P}_{\text {mv }}$. This leads to the optimization problem shown below

$$
\hat{\mathrm{a}}_{\mathrm{MV}}=\max _{\mathbf{a}}\left\{\mathcal{P}_{\mathrm{mv}}\right\}=\max _{\mathbf{a}}\left\{\frac{1}{\mathrm{a}^{\dagger} \mathrm{E}^{\dagger} \mathrm{S}^{-1} \mathrm{Ea}}\right\} \text { subject to } \mathbf{a}^{\dagger} \mathbf{a}=1
$$

The constraint eliminates the trivial solution where $\hat{\mathrm{a}}_{\mathrm{MV}}$ is equal to the zero vector. Note that $\widehat{a}_{\mathrm{mv}}$ is an estimate of the relative weights, normalized by $\widehat{a}_{\mathrm{mv}}^{\dagger} \widehat{a}_{\mathrm{mv}}=1$; the true scaling associated with the signal must be obtained from the power estimate. Equation 4.6 is a well-known quadratic optimization problem $[39,40] .{ }^{1}$ The following eigenvalue equation determines the stationary points of $\mathcal{P}_{\mathrm{mv}}$,

$$
\mathrm{E}^{\dagger} \mathrm{S}^{-1} \mathrm{Ey}=\nu \mathrm{y}
$$

\footnotetext{
${ }^{1}$ Most linear algebra texts discuss this problem in the context of the Rayleigh quotient. The same results can be obtained using the concept of matrix pencils. See the development by Gantmacher [40].
} 
The eigenvectors, $\mathbf{y}$, are normalized to have unit length. The maximum value of $\mathcal{P}_{\mathrm{mv}}$

corresponds to $\nu_{\min }^{-1}$ and the maximizing distribution a is the eigenvector $\mathrm{y}_{\min }$. According to Section 2.3, these two parameters completely describe the correlation matrix for coherent modes: (1) $\mathcal{P}_{\text {mv }}$ represents an estimate of the total power in the signal, and (2) $\widehat{\mathrm{a}}_{\mathrm{mv}}$ contains the relative power distribution (See Eq. 2.17). Therefore the minimum variance estimate of $\mathrm{S}_{\mathrm{M}}$ becomes

$$
\hat{\mathrm{S}}_{\mathrm{M} \_\mathrm{MV}}=\frac{1}{\nu_{\min }} \mathrm{y}_{\min } \mathrm{y}_{\min }^{\dagger} \text {. }
$$

In order to illustrate the basic characteristics of the minimum variance modal estimator, it is useful to revisit the first deep water example given in Chapter 3. A detailed performance analysis for the MV method follows in Sections 4.1.1-4.1.4.

\section{Deep Water Example}

The simulation environment is the deep water waveguide that is sampled with the 40 element vertical array described in Chapter 2. Measured pressure consists of the test signal (defined on page 36) plus white sensor noise. Ideal sensor covariance matrices are used in this example. The processing attempts to resolve the power in the first 10 modes. Each plot includes the least squares results for comparison purposes.

Figure 4-1 shows the ideal MV estimates of the modal powers for three different signalto-noise ratios: $10 \mathrm{~dB}, 0 \mathrm{~dB}$, and $-10 \mathrm{~dB}$. Circles denote the true power distribution which is an alternating pattern $(0 \mathrm{~dB}$ to $-10 \mathrm{~dB})$ for modes $1-8$. Modes 9 and 10 are not included in the test signal. At $10 \mathrm{~dB}$ SNR (top plot), the LS and MV estimates are virtually identical for the lowest 8 modes. However, the estimates for modes 9 and 10 indicate that the minimum variance processor has a significant advantage over least squares in maintaining nulls in the modal power spectrum. The MV estimates for these modes are down more than $90 \mathrm{~dB}$ from the reference level, while the LS estimates are down only $20 \mathrm{~dB}$. For the lower SNR's shown in the bottom two plots, the minimum variance estimator keeps the nulls although the estimated levels increase slightly. In addition for modes 1-8, the MV processor exhibits very little of the bias error that corrupts the LS estimate at $0 \mathrm{~dB}$ and $-10 \mathrm{~dB}$ SNR.

In order to understand the behavior shown in Fig. 4-1, consider the eigenvalues and eigenvectors of $\mathbf{E}^{\dagger} \mathrm{S}^{-1} \mathrm{E}$ for the ideal covariance case. The environmental model for the deep water example includes a coherent modal signal plus white sensor noise. In this case 

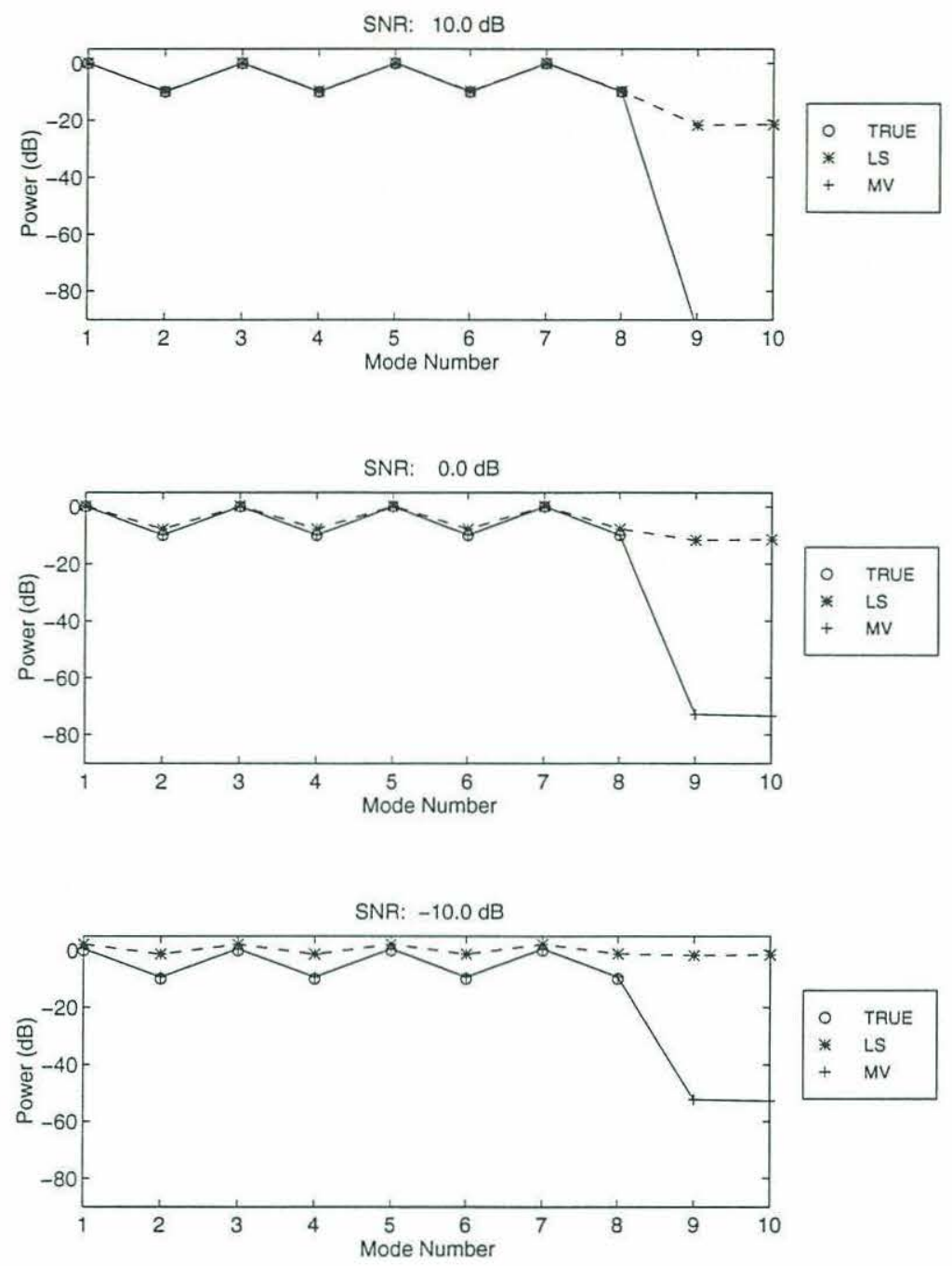

Figure 4-1: Minimum variance power estimates for the deep water example

the sensor covariance matrix is given by

$$
\mathbf{S}=\mathcal{P}_{\mathrm{T}} \mathrm{Ea}_{\mathrm{T}} \mathbf{a}_{\mathrm{T}}^{\dagger} \mathbf{E}^{\dagger}+\sigma_{w}^{2} \mathrm{I}
$$

The minimum variance method requires the inverse of $\mathbf{S}$ which can be determined using the Woodbury identity (or matrix inversion lemma) [41],

$$
\mathrm{S}^{-1}=\frac{1}{\sigma_{w}^{2}}\left[\mathrm{I}-\left(\frac{\mathcal{P}_{\mathrm{T}} / \sigma_{w}^{2}}{1+\left(\mathcal{P}_{\mathrm{T}} / \sigma_{w}^{2}\right)\left(\mathrm{a}_{\mathrm{T}}^{\dagger} \mathrm{E}^{\dagger} \mathrm{Ea}_{\mathrm{T}}\right)}\right) \mathrm{Ea}_{\mathrm{T}} \mathrm{a}_{\mathrm{T}}^{\dagger} \mathrm{E}^{\dagger}\right]
$$


Substituting Eq. 4.10 into Eq. 4.7, the MV eigenvalue problem becomes

$$
\frac{1}{\sigma_{w}^{2}}\left[\mathrm{E}^{\dagger} \mathrm{E}-\left(\frac{\mathcal{P}_{\mathrm{T}} / \sigma_{w}^{2}}{1+\left(\mathcal{P}_{\mathrm{T}} / \sigma_{w}^{2}\right)}\left(\mathbf{a}_{\mathrm{T}}^{\dagger} \mathrm{E}^{\dagger} \mathrm{E} \mathrm{a}_{\mathrm{T}}\right)\right) \mathrm{E}^{\dagger} \mathrm{Ea}_{\mathrm{T}} \mathbf{a}_{\mathrm{T}}^{\dagger} \mathrm{E}^{\dagger} \mathrm{E}\right] \mathrm{y}=\nu \mathrm{y} .
$$

Since the general form of Eq. 4.11 does not lead to an analytically tractable solution, the following development assumes that the sampled modeshapes are orthogonal. In this case, the modeshape correlation matrix is a multiple of the identity matrix i.e., ${ }^{2}$

$$
\mathbf{E}^{\dagger} \mathbf{E}=\beta \mathbf{I}
$$

This simplifying assumption permits an analytical solution which provides significant insights about the minimum variance processor. For the deep water example, this assumption is relatively justified because the 10 estimated modes are adequately sampled by the simulation array (refer to Fig. 2-6). Section 4.1.2 addresses the issue of non-orthogonal modes.

Assuming that the sampled modes are orthogonal, Eq. 4.11 becomes,

$$
\frac{\beta}{\sigma_{w}^{2}}\left[\mathbf{I}-\left(\frac{\beta \mathcal{P}_{\mathrm{T}} / \sigma_{w}^{2}}{1+\beta \mathcal{P}_{\mathrm{T}} / \sigma_{w}^{2}}\right) \mathbf{a}_{\mathrm{T}} \mathbf{a}_{\mathrm{T}}^{\dagger}\right] \mathbf{y}=\nu \mathrm{y}
$$

From this equation it is trivial to show that $\mathrm{a}_{\mathrm{T}}$ is an eigenvector of $\mathrm{E}^{\dagger} \mathbf{S}^{-1} \mathbf{E}$ with the corresponding eigenvalue $\nu=\frac{\beta}{\sigma_{w}^{2}}\left(\frac{1}{1+\beta \mathcal{P}_{\mathrm{T}} / \sigma_{w}^{2}}\right)$. Using the property that eigenvectors of symmetric matrices are orthonormal, it can be shown that all other eigenvalues of $\mathrm{E}^{\dagger} \mathrm{S}^{-1} \mathrm{E}$ are equal to $\frac{\beta}{\sigma_{w}^{2}}$. Thus, the eigenvalue corresponding to $\mathrm{a}_{\mathrm{T}}$ is the minimum eigenvalue. This leads to the ideal power and modal distribution estimates shown below

$$
\mathcal{P}_{\mathrm{mv}}=\nu_{\min }^{-1}=\mathcal{P}_{\mathrm{T}}+\frac{\sigma_{w}^{2}}{\beta}
$$

and

$$
\mathrm{a}_{\mathrm{mv}}=\mathrm{y}_{\min }=\mathrm{a}_{\mathrm{T}} \text {. }
$$

The minimum variance power estimate is obviously biased due to the white noise incident on the array. A reasonable way to eliminate this bias might be to subtract the inverse of the maximum eigenvalue $\left(\nu_{\max }=\sigma_{w}^{2} / \beta\right)$ from $\mathcal{P}_{\mathrm{mv}}$. In the ideal-covariance, orthogonal-mode

\footnotetext{
${ }^{2}$ This argument implicitly assumes that the modes are scaled equally. Note that $\beta$ is a scale factor that depends on the number of sensors: $0<\beta \leq 1$.
} 
case, the bias correction is clearly justified. When the covariance matrix must be estimated from snapshot data or when the modes are not orthogonal, the eigenstructure of $\mathrm{E}^{\dagger} \mathrm{S}^{-1} \mathrm{E}$ is more complicated. In these cases bias correction is not feasible. For this reason, the rest of the MV analysis concerns the biased power estimate.

Based on Equations 4.14 and 4.15, the minimum variance estimate of the mode correlation in the ideal case is ${ }^{3}$

$$
\mathrm{S}_{\mathrm{M}_{-} \mathrm{MV}}=\left(\mathcal{P}_{\mathrm{T}}+\frac{\sigma_{w}^{2}}{\beta}\right) \mathbf{a}_{\mathrm{T}} \mathbf{a}_{\mathrm{T}}^{\dagger}=\mathrm{S}_{\mathrm{M}}+\frac{\sigma_{w}^{2}}{\beta} \mathbf{a}_{\mathrm{T}} \mathbf{a}_{\mathrm{T}}^{\dagger}
$$

According to the above equation, the MV processor biases the power in each mode by a constant term which depends on the white noise level and the true power in the mode. As a result, modes which are not present in the signal should not be biased. The plots in Figure 4-1 support this conclusion since the MV estimates of modes 9 and 10 show very little bias.

Figure 4-2 shows the error in the MV power estimates for the deep water example. The error vector $\mathbf{e}$ is defined as the absolute value of the differences in the true powers and the estimated powers (see Eq. 2.29). The figure shows errors on a $\log$ scale $\left(10 \log _{10} \mathrm{e}_{j}\right)$ for the three SNR levels used in the example. The plots reveal that the MV errors are proportional to the true powers, as predicted by Eq. 4.16. (Recall that modes 1-8 alternate between $0 \mathrm{~dB}$ and $-10 \mathrm{~dB}$ and that modes 9 and 10 are not present.) An increase in the noise power $\left(\sigma_{w}^{2}\right)$, which is reflected as a decrease in SNR, merely shifts the error curves up by a constant amount. Note that the errors for minimum variance are always lower than the constant errors for the least squares estimate.

Based on this example, the minimum variance estimator has several favorable characteristics which merit further research. In particular, the ability of the estimator to maintain nulls in the spectrum is especially useful in circumstances where there is little a priori knowledge of the power distribution. The rest of this section focuses on analysis of the MV estimator with respect to the issues outlined in Section 2.3. Each subsection draws appropriate comparisons between the performance of the LS and MV methods.

\footnotetext{
${ }^{3}$ Reminder: $\mathrm{S}_{\mathrm{M}_{-} \mathrm{MV}}$ is the ideal covariance result and $\widehat{\mathrm{S}}_{\mathrm{M} \_\mathrm{MV}}$ is the estimated covariance result.
} 

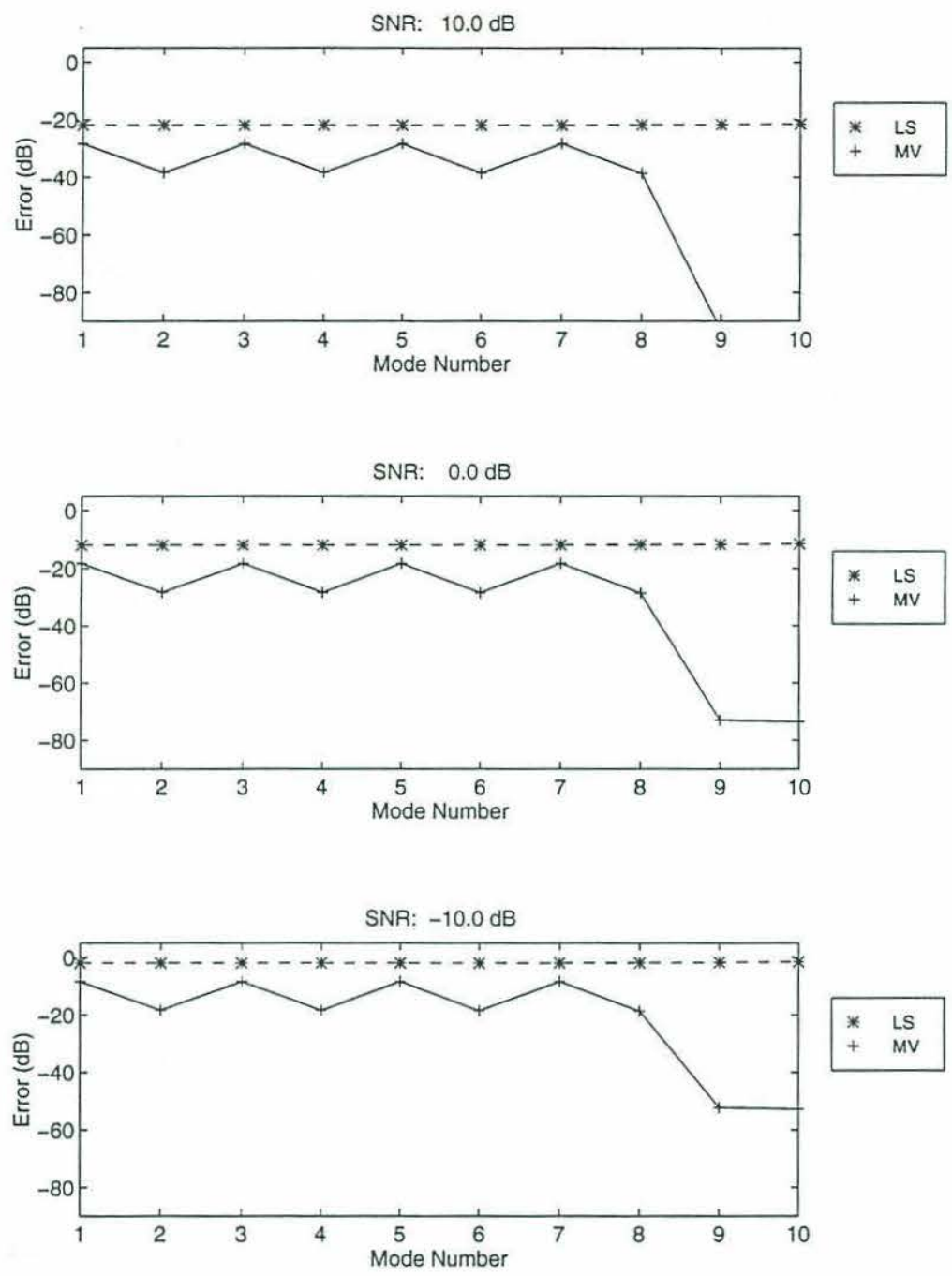

Figure 4-2: Error in minimum variance power estimates for the deep water example

\subsubsection{Ambient Noise}

The first issue to consider in the performance evaluation is the effects of ambient noise on the estimates. Instead of ignoring the noise, as LS algorithms do, the MV formulation uses an adaptive method to reduce its effects.

Based on Equation 4.16, the error in mode $j$ for the MV estimator is proportional to the white noise power and the relative power in the mode, i.e.,

$$
e_{j}=\frac{\sigma_{w}^{2}}{\beta}\left[\mathrm{a}_{\mathrm{T}} \mathrm{a}_{\mathrm{T}}^{\dagger}\right]_{j j} .
$$

Note that the above expression for the error depends on the modes being orthogonal 
(i.e., $\mathbf{E}^{\dagger} \mathbf{E}=\beta \mathrm{I}$ ). According to Equation 4.17, the MV error is a linear function of the noise and is scaled by the true power in the mode.

Consider an example. The salient parameters are identical to the deep water environment described above with the exception that the white noise power is adjusted so that the SNR varies from $-20 \mathrm{~dB}$ to $20 \mathrm{~dB}$. Ideal sensor covariances are used to generate the results. Figure $4-3$ shows the $\log$ of the error vs. SNR for modes 1,2 , and 10. The MV
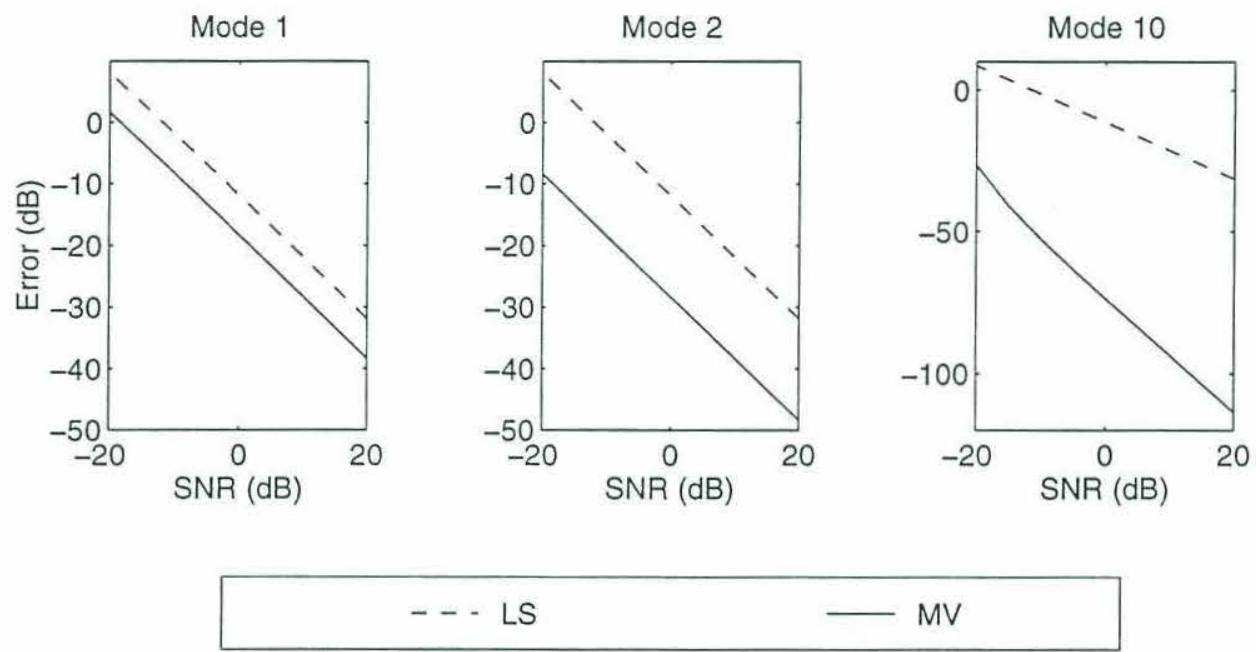

Figure 4-3: Error vs. SNR for modes 1, 2, and 10 of the deep water waveguide. Ambient noise consists of white sensor noise only. Both MV and LS errors are shown for the ideal covariance case. Note that the vertical scale for mode 10 is different than for modes 1 and 2 .

errors for modes 1 and 2 are linear functions of the SNR, and the error for mode 2 is lower than for mode 1 . These results agree with the predicted errors since modes 1 and 2 are excited at levels of $0 \mathrm{~dB}$ and $-10 \mathrm{~dB}$, respectively. Recall that mode 10 is not contained in the test signal, therefore according to Equation 4.17 the error for mode 10 should be zero $(-\infty \mathrm{dB})$. While the figure indicates that the error for mode 10 is significantly lower than the other errors, it is clearly not zero. This deviation from predicted behavior can be attributed to the fact that the sampled modeshapes are not perfectly orthogonal. Note that the performance of the Ferrara/Parks estimator is always better than least squares. In the worst case (mode 1 ), the MV estimation error is consistently $\approx 8 \mathrm{~dB}$ lower than the LS error. In the best case (mode 10), the difference in the MV and LS errors is on the order of $40 \mathrm{~dB}$. (A different vertical scale is used in the error plot for mode 10.)

Recall from Chapter 3 that the least squares processor is incapable of resolving multiple modal signals. It is worthwhile to note that the Ferrara/Parks formulation has the potential 
to estimate the powers associated with several sources. The basic idea is that multiple discrete sources are associated with multiple peaks in the power spectrum, therefore the modal signals can be separated. This aspect of the Ferrara/Parks processor is an interesting area for further research.

\subsubsection{Modal Orthogonality}

Since the analysis of the MV algorithm depends on the orthogonality of the sampled modeshapes, it is imperative to clarify the number of modes that can be accurately estimated using the Ferrara/Parks minimum variance technique.

Consider using the 40-element simulation array to estimate the average powers in the first 16 modes of the deep water waveguide. The sampled modeshapes for these modes are not orthogonal. One indication of the loss of orthogonality is that $D O F_{\text {eff }}$ is only equal to 14 when $\mathrm{E}$ includes 16 modes. Assume that the input signal consists of an alternating pattern of the first 16 modes: odd modes are excited at $0 \mathrm{~dB}$ and even modes excited at $-10 \mathrm{~dB}$. The white noise level is fixed so that the average SNR is $0 \mathrm{~dB}$ for this example. The number of data snapshots is not an issue since ideal sensor covariances are used. Figure 44 shows the error vector $\left(10 \log _{10} \mathbf{e}_{j}\right)$ for the 16 -mode estimate. The loss of two degrees

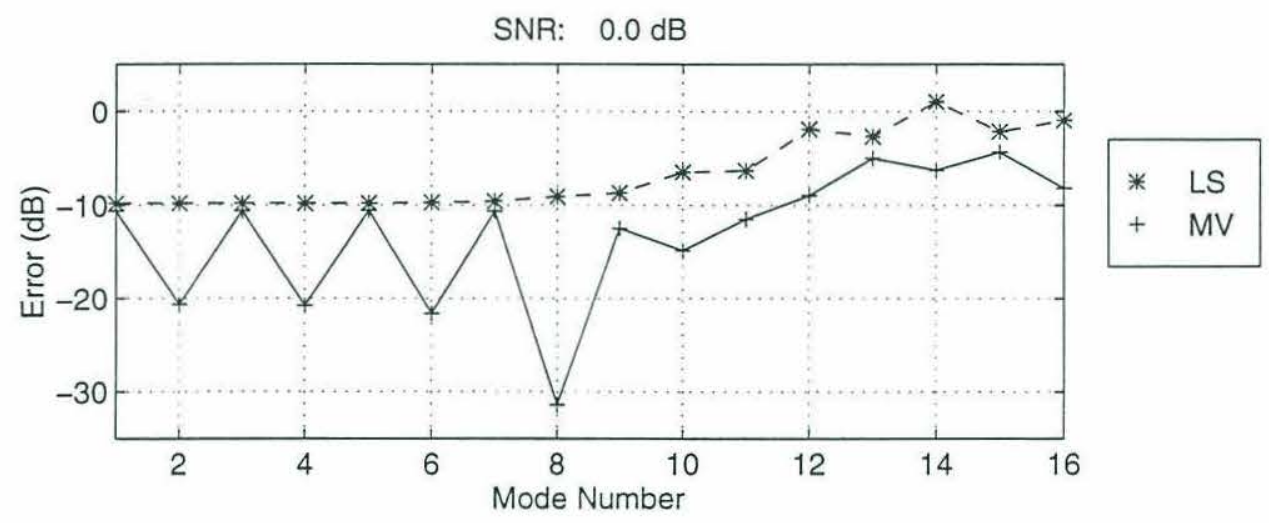

Figure 4-4: Error vector for the 16-mode example using the deep water waveguide

of freedom in $\mathrm{E}^{\dagger} \mathrm{E}$ clearly has an effect on the results. While the MV error for modes 1-8 follows the expected alternating pattern, the errors for the higher order modes do not follow a predictable pattern. Note that the MV estimation error is always below the LS error.

Next, consider how the error varies as a function of the number of modes included in the estimate. The parameters of this example are identical to the first deep water 
example of this chapter, i.e., the test signal consists of the lowest 8 modes in an alternating $0 /-10 \mathrm{~dB}$ pattern. The number of modes to estimate is varied from 8 up to 16 . Recall that a useful scalar performance measure is the total error defined in Chapter 2, e.g., for the MV estimator

$$
\text { Total Error }=\operatorname{TR}\left\{\left|\widehat{S}_{M_{-} M V}-S_{M}\right|\right\} \text {. }
$$

Figure 4-5 shows total error vs. number of modes to estimate for two different signal-to-noise ratios. The least squares results are shown for comparison purposes. For the $0 \mathrm{~dB}$ SNR
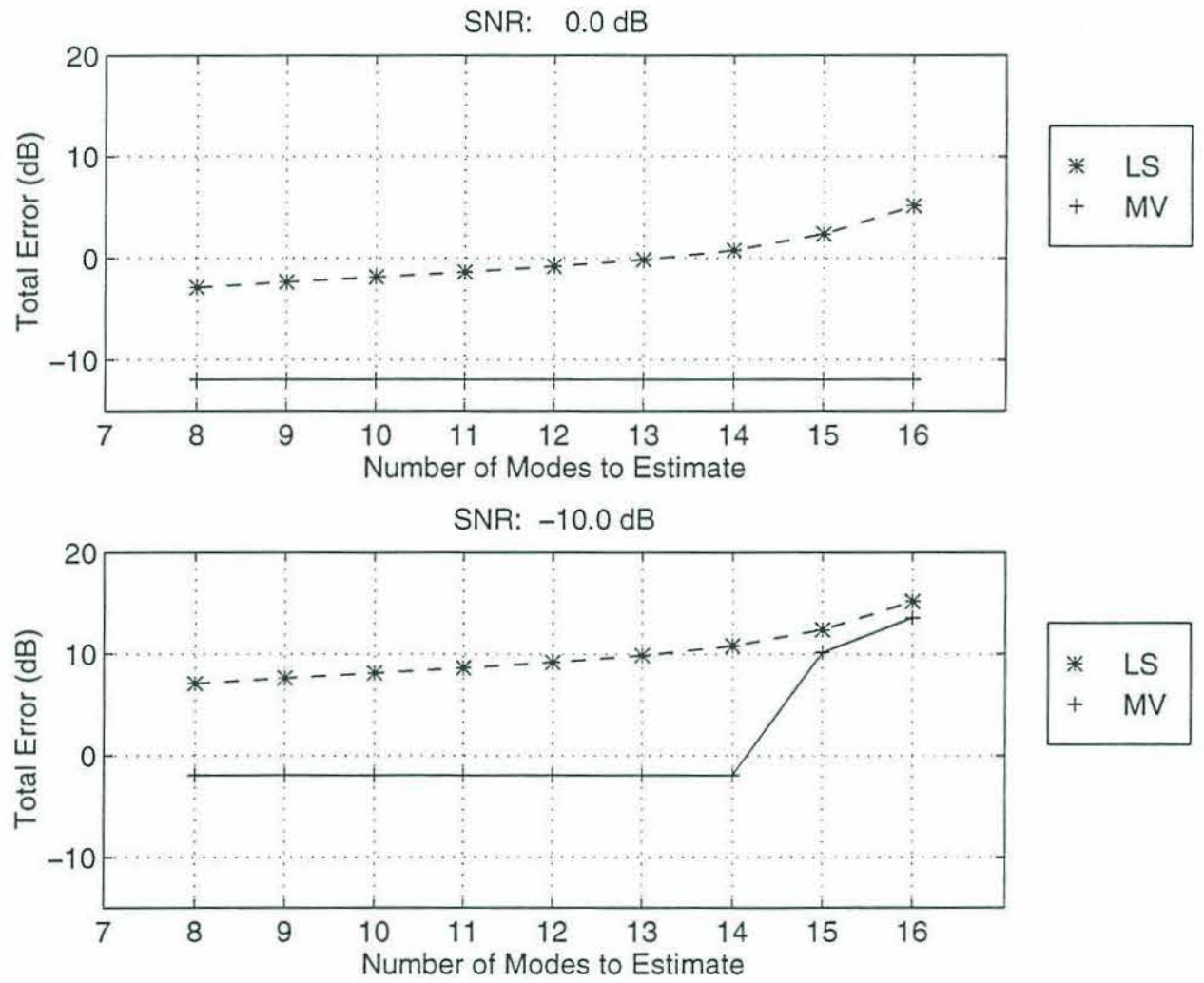

Figure 4-5: Total error vs. number of modes to estimate for the deep water example. Two cases are shown: the top plot displays the $0 \mathrm{~dB}$ SNR results and the bottom plot displays the $-10 \mathrm{~dB}$ SNR results.

case, the MV error is constant, implying that the loss of degrees of freedom in $\mathrm{E}^{\dagger} \mathrm{E}$ has little effect on this formulation. At -10 dB SNR, however, the MV error is constant until the number of modes reaches 15 when it jumps to a value close to the LS result. Note that for 15 modes, $\mathrm{E}^{\dagger} \mathrm{E}$ has lost more than one degree of freedom (see Figure 2-7). From this example it appears that low orthogonality combined with low SNR has adverse effects on the accuracy of the MV estimates. 
It is interesting to examine what happens to the MV estimate for the 15-mode case at $-10 \mathrm{~dB}$ SNR. The solid line in Figure 4-6 represents the MV estimate of the powers in the first 15 modes of the deep water waveguide. In other words, the solid line corresponds to the eigenvector associated with the maximum peak in the MV spectrum. The dashed line represents the eigenvector corresponding to the second highest power (i.e., second smallest eigenvalue). It appears that the MV algorithm has essentially chosen the wrong peak since

the second eigenvector is actually a better approximation to the true modal power spectrum (denoted by circles). Figure 4-7 is a plot of the stationary points of the MV spectrum (i.e., the inverse of the eigenvalues) for both $0 \mathrm{~dB}$ and $-10 \mathrm{~dB}$ SNR. In the $0 \mathrm{~dB}$ case the second highest power is on the order of $10 \mathrm{~dB}$ lower than the peak. For the $-10 \mathrm{~dB}$ case, however, the two highest peaks are with approximately $3 \mathrm{~dB}$ of each other. In theory there should be only one peak corresponding to the signal and a set of constant values that are proportional to the white noise. The purpose of this example is to illustrate that a combination of low SNR and decreased orthogonality can lead to a global localization problem for the MV estimator. Note that it is not always possible to obtain a more accurate estimate by looking at the other eigenvectors associated with the stationary points of the power spectrum. The eigenvalue spectrum, however, is a valuable indicator of potential localization errors. If the stationary points show one dominant peak, then the estimator has probably located the correct vector of relative mode amplitudes. If not, there are two possibilities. First, the noise and decreased orthogonality are severely corrupting the estimates. One solution to this problem is to reduce the number of modes included in the estimate. A second possibility is that the signal is not coherent; recall that the derivation of the Ferrara/Parks MV algorithm specifically assumes a coherent signal model. Chapter 5 proposes a method for dealing with incoherent modes.

\subsubsection{Estimated Covariance Issues}

The preceding examples have used ideal sensor covariance matrices for the simulated data, but in practice the MV processor must use the sample covariance matrix $\widehat{\mathrm{S}}$. As a result the MV modal power estimates are actually random variables and can be characterized by their second order statistics. Appendix $\mathrm{C}$ derives the mean and variance of the MV power estimates using known characteristics of $\widehat{\mathrm{S}}$ and the minimum variance spectral estimator. The results are summarized below. 


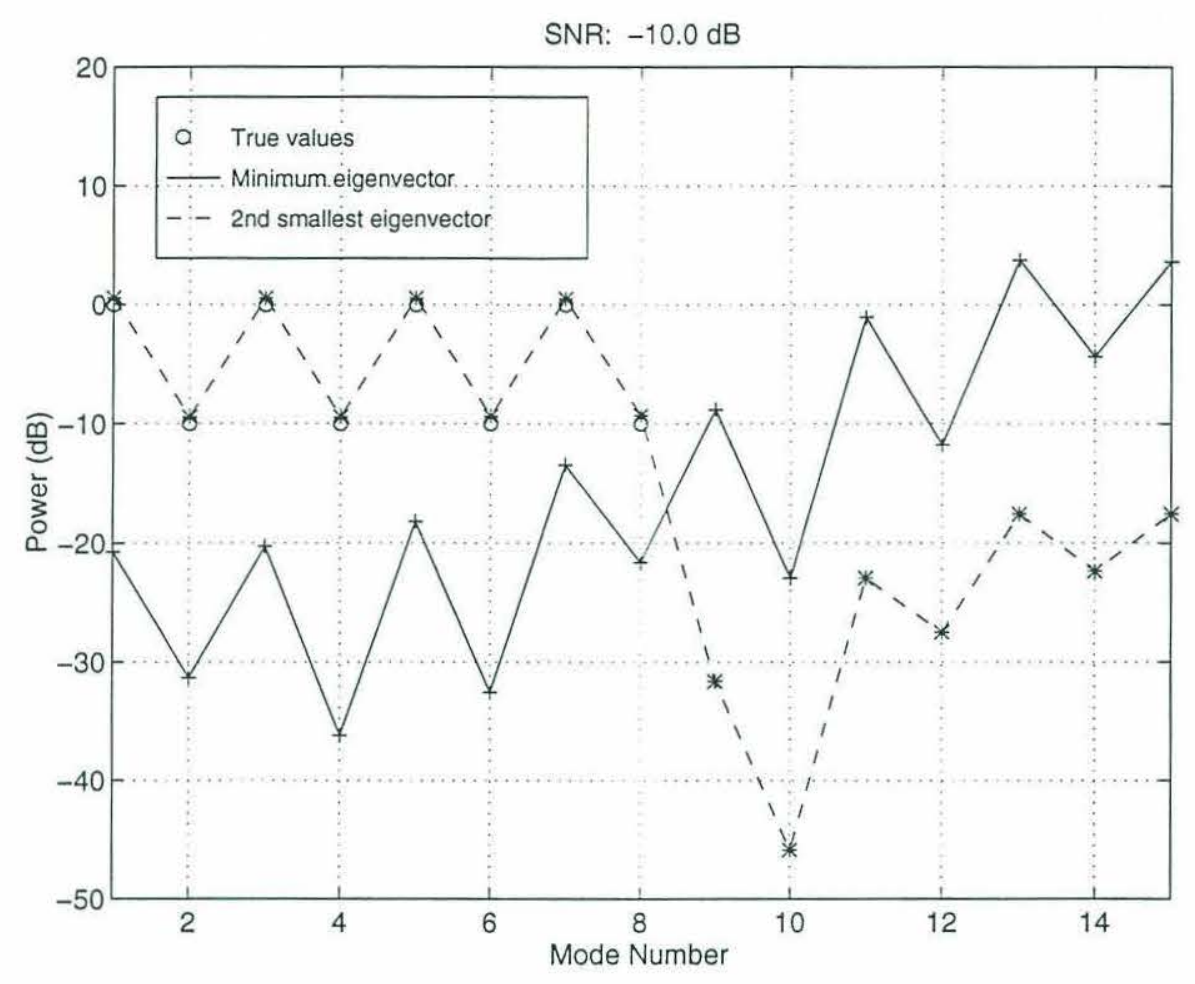

Figure 4-6: Example of global localization problem for the MV estimator: 15 modes estimated at $-10 \mathrm{~dB}$ SNR.

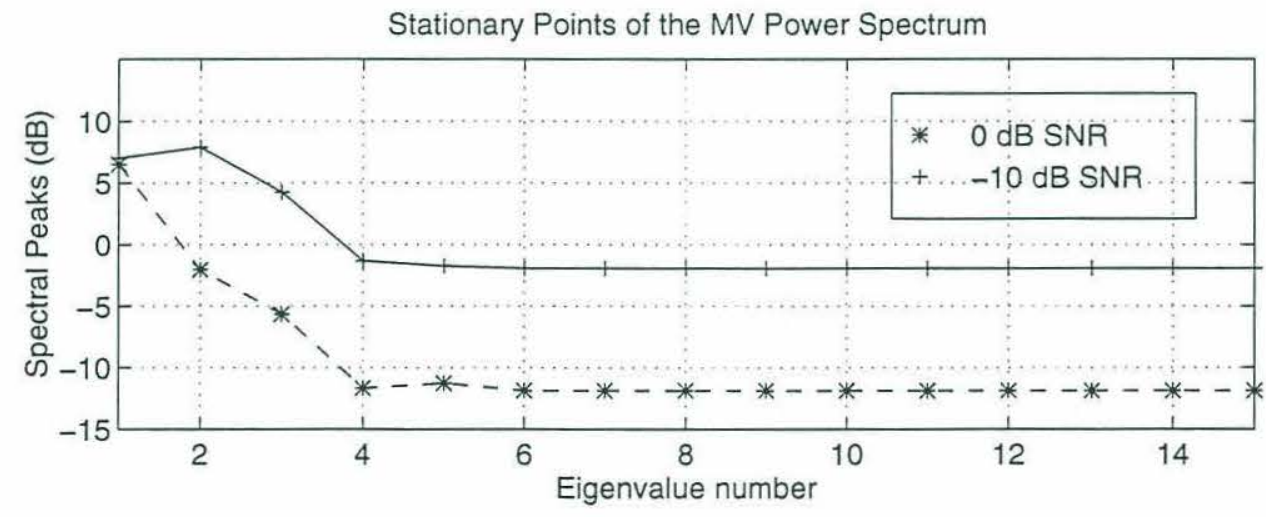

Figure 4-7: Peaks of the minimum variance power spectrum for the 15-mode estimate at two SNR levels: $0 \mathrm{~dB}$ and $-10 \mathrm{~dB}$. 
An assumption is necessary in order to derive simple expressions for the bias and variance associated with the MV estimates. Specifically, the following development assumes that the optimization in the Ferrara/Parks formulation introduces no errors, i.e., that the estimate of the relative modal distribution $\widehat{\mathrm{a}}_{\mathrm{mv}}$ is correct. In this case the only errors in the MV results are due to the power estimate $\widehat{\mathcal{P}}_{\text {mv }}$. Without this simplification, the calculations are difficult because they require a thorough analysis of the eigenvalue problem in Eq. 4.7 with respect to estimatated covariance issues.

Consider the case of a modal signal plus white sensor noise. Based on work by Capon and Goodman [22], the standard minimum variance power estimate has a bias associated with the number of data snapshots. As discussed in Appendix C, a correction for the snapshot bias is feasible. The results of this section assume that the correction has been implemented. In this case the expected power in mode $j$ can be written

$$
\mathcal{E}\left\{\widehat{P}_{j \_\mathrm{mv}}\right\} \equiv \bar{P}_{j \_\mathrm{mv}}=P_{j}+\frac{\sigma_{w}^{2}}{\beta}\left[\mathbf{a}_{\mathrm{T}} \mathbf{a}_{\mathrm{T}}^{\dagger}\right]_{j j}
$$

where $P_{j}$ is the true power in mode $j$. As derived in the appendix, the variance for the MV power estimate for mode $j$ is shown below

$$
\operatorname{Var}\left\{\widehat{P}_{j \_\mathrm{mv}}\right\}=\frac{\left(\bar{P}_{j \_\mathrm{mv}}\right)^{2}}{L-N+1} .
$$

$L$ is the number of data snapshots and $N$ is the number of array elements.

Based on these two equations, it is possible to predict bias and variance for the MV estimates. The following example contrasts the predictions with the results of a Monte Carlo simulation. This is useful in determining the validity of the assumption used to derive the bias and variance. The parameters of the simulation are identical to the deep water example using the test signal. Each data point is the result of averaging over 1000 independent trials. Figure 4-8 shows the mode 1 error vs. the number of snapshots. Both predicted and Monte Carlo errors for the MV and LS processors are shown. The MV estimator is clearly affected by the number of snapshots available to estimate the covariance. In fact the average error in the MV estimate for mode 1 does not fall below the LS result until the number of data snapshots exceeds 230. Note that the predicted error for the minimum variance estimate is constant. The difference between the predicted and Monte Carlo errors 
Mode 1 Error: $0 \mathrm{~dB}$ SNR

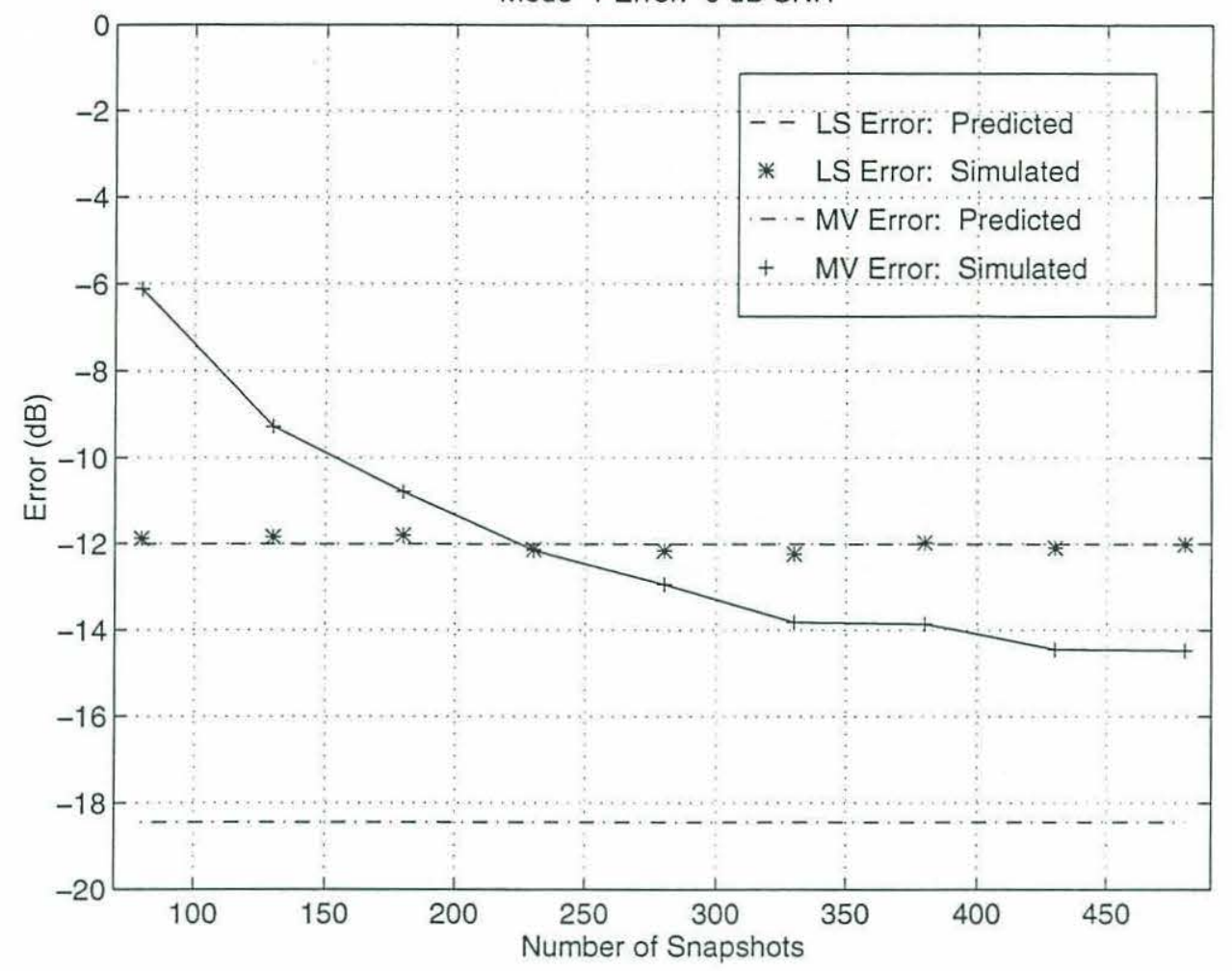

Figure 4-8: Bias error vs. number of snapshots for mode 1. Predicted and Monte Carlo results are shown.

can be attributed to the fact that the estimates of the relative modal distribution (i.e., the minimum eigenvector) improve as the estimate of the sensor covariance gets more accurate. (Recall that the derivation of the predicted error assumes that $\widehat{\mathrm{a}}_{\mathrm{mv}}$ is always estimated correctly.)

Figure 4-9 shows the variance vs. number of snapshots for the MV estimator. The predicted and Monte Carlo simulation values are shown, along with the Cramer Rao bound. Based on Appendix A, the Cramer Rao bound on the variance goes as $\frac{1}{L}$, whereas the MV estimator variance is a function of $\frac{1}{L-N+1}$. These differences are obvious from the plot. Note that the simulation results indicate a slightly higher variance than the predicted values. This is due to the fact that the derivation of the MV variance does not account for the uncertainties associated with the eigenvalue problem. 


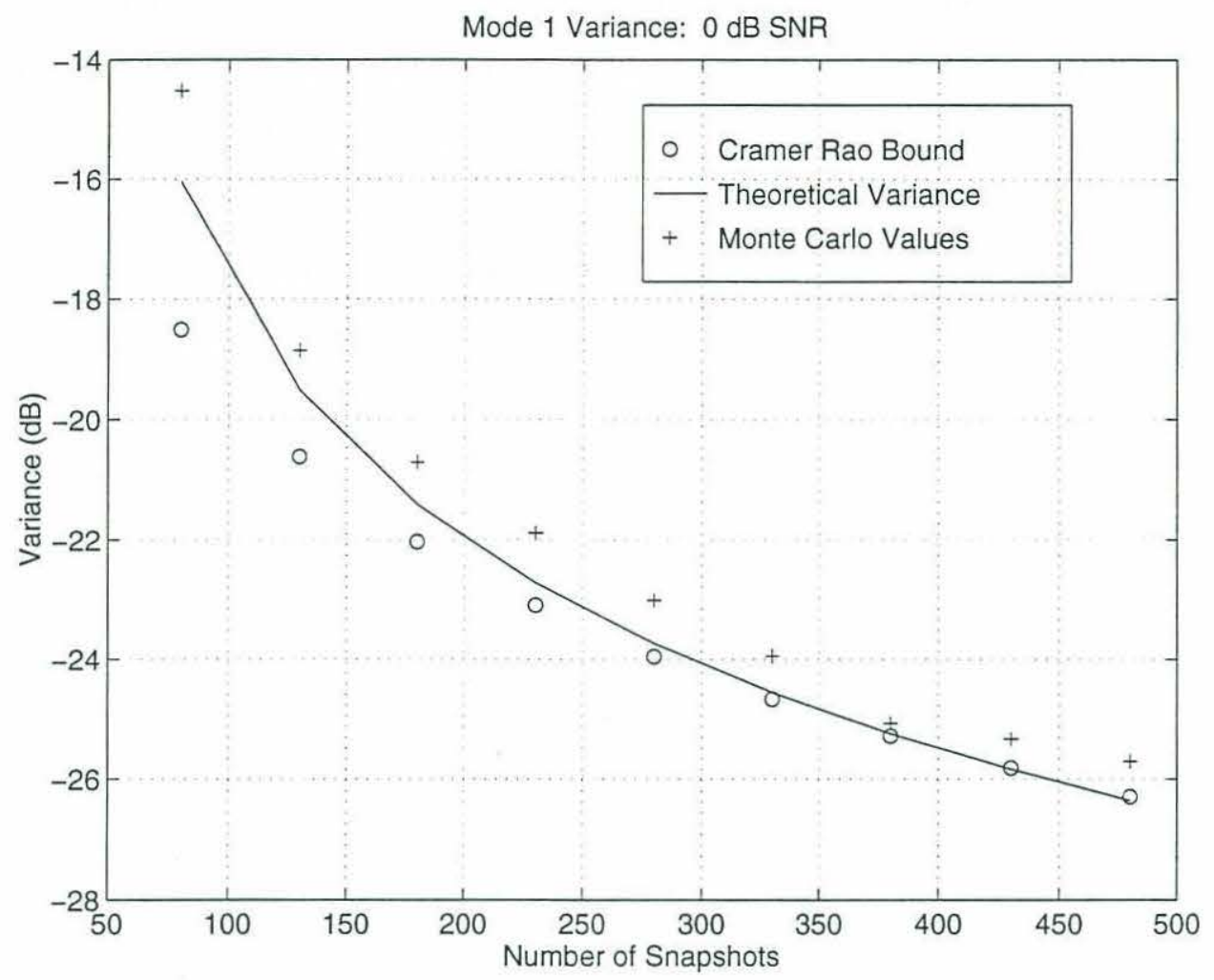

Figure 4-9: Variance vs. number of snapshots for the MV estimator. Predicted and Monte Carlo simulation values are shown. The Cramer Rao bound is given for reference.

\subsubsection{Signal Coherence}

The derivation of the MV Ferrara/Parks processor explicitly assumes that the modal signal is coherent. As a result, performance degrades significantly if the incident signal loses coherence. A simple example illustrates this point.

The signal model is based on the adiabatic channel with internal waves described in Chapter 2. The form of the mode correlation matrix is shown below

$$
\mathrm{S}_{\mathrm{M}}=(1-\gamma) \operatorname{diag}\left[\mathrm{x}_{0} \mathbf{x}_{0}^{\dagger}\right]+\gamma \mathbf{x}_{0} \mathbf{x}_{0}^{\dagger} \quad 0 \leq \gamma \leq 1
$$

Recall that the vector $\mathrm{x}_{0}$ contains the deterministic mode amplitudes and $\gamma$ is the coherence parameter. For $\gamma=0$ the signal consists of a totally incoherent sum of modes and for $\gamma=1$ the sum is perfectly coherent.

For this example the simulation environment is the deep water waveguide. The signal has the same modal content as the test signal used earlier, but the coherence level is varied from 0 to 1 . Again, additive noise in the environment consists of white sensor noise only. 
The noise power is set so that the input SNR is equal to $0 \mathrm{~dB}$. The 40 -element simulation array samples the pressure field. The purpose of the processing is to resolve the first 10 modes of the waveguide. Ideal sensor covariance matrices are used in this example, therefore the number of data snapshots is not an issue.

Figure 4-10 shows the total error in the MV estimate as a function of coherence. Least squares results are shown for comparison. Recall that one of the advantages of the LS

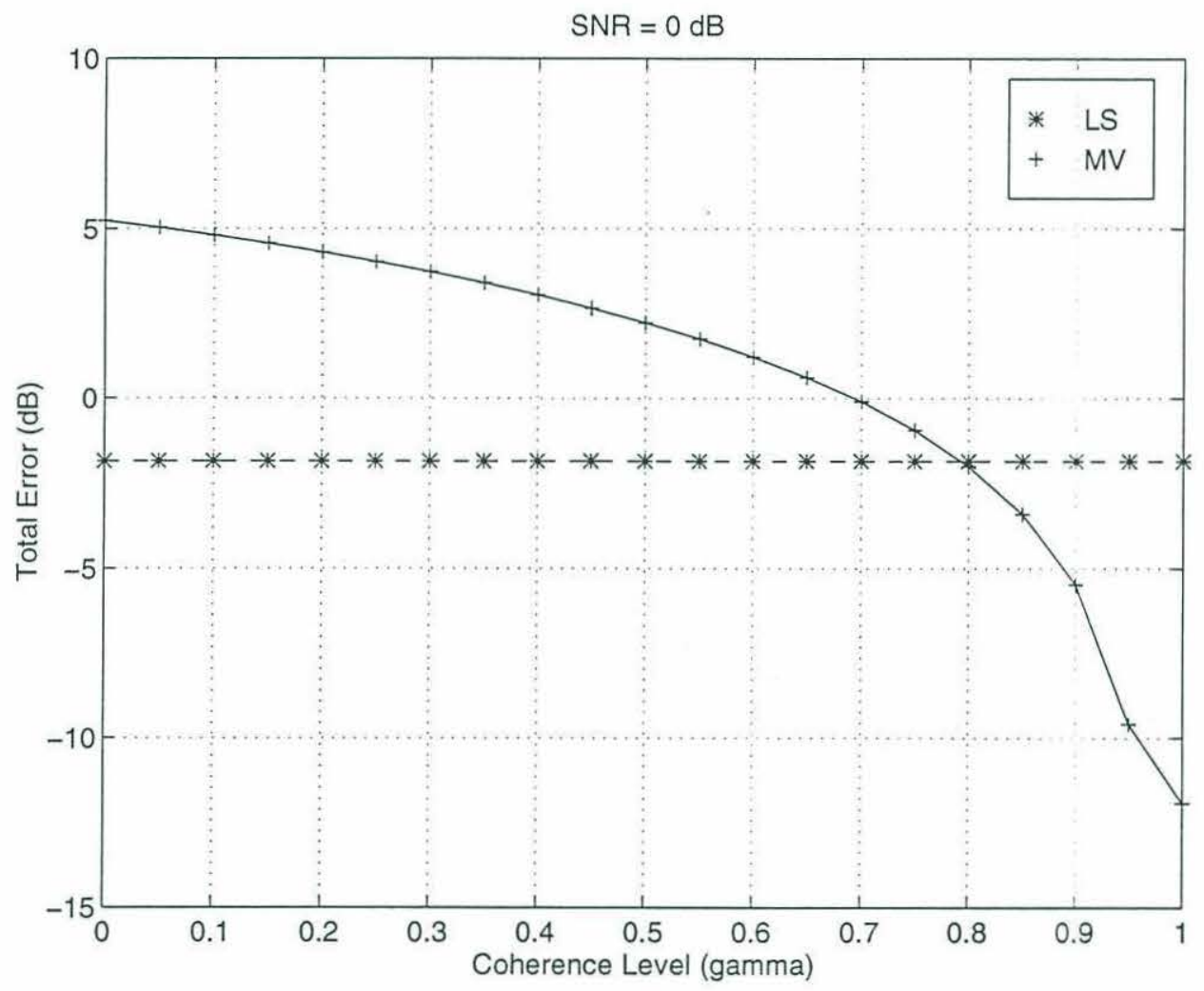

Figure 4-10: Total error vs. coherence for the Ferrara/Parks MV formulation

estimator is that it does not rely on the coherence of the signal, hence the LS error is constant with respect to $\gamma$. As expected, the MV algorithm performs poorly when the signal is not perfectly coherent. At $0 \mathrm{~dB}$ SNR it appears that the Ferrara/Parks approach incurs larger errors than the conventional method unless $\gamma>0.8$. The new adaptive MV processor introduced in this section is designed specifically for coherent modes and is not useful when signal coherence is reduced. 


\subsection{MUSIC Modal Estimator}

MUltiple SIgnal Classification, commonly known as MUSIC, is one of a family of algorithms referred to as subspace methods. The purpose of these methods is to resolve the discrete components of the power spectrum. For time series analysis this consists of estimating the frequency and power of complex exponentials in additive noise. In array processing these algorithms are most often used to estimate the angle-of-arrival (AOA) of a coherent signal against a noisy background. The work of Pisarenko [42] on estimation of complex exponentials in white noise stimulated the development of subspace methods. R.O. Schmidt introduced the MUSIC algorithm in 1981 [36]. The technique has gained broad acceptance, and as a result the literature is full of variations and improvements, e.g., ESPRIT [37].

Subspace methods rely on separating data into signal and noise subspaces using an eigenvector decomposition of the covariance matrix,

$$
\mathbf{S}=\underbrace{\sum_{i=1}^{N_{s}} \lambda_{i} \mathbf{v}_{i} \mathbf{v}_{i}^{\dagger}}_{\text {signal subspace }}+\underbrace{\sum_{i=N_{s}+1}^{N} \lambda_{i} \mathbf{v}_{i} \mathbf{v}_{i}^{\dagger}}_{\text {noise subspace }} .
$$

For array processing applications, Equation 4.22 assumes an $N$-element array with $N_{s}$ separate signals impinging on it. To understand why this decomposition is useful, consider the case of a signal plus white noise. Let $\mathrm{g}$ be the normalized $\left(\mathrm{g}^{\dagger} \mathrm{g}=1\right)$ replica vector associated with the signal. The sensor covariance is

$$
\mathrm{S}=\mathcal{P}_{g} \mathrm{gg}^{\dagger}+\sigma_{w}^{2} \mathrm{I}
$$

where $\mathcal{P}_{g}$ and $\sigma_{w}^{2}$ are the signal and noise powers, respectively. It is trivial to show that $\mathrm{g}$ is an eigenvector of $\mathbf{S}$, corresponding to the eigenvalue $\lambda=\mathcal{P}_{g}+\sigma_{w}^{2}$. Since the eigenvectors of a symmetric matrix (such as $\mathbf{S}$ ) form an orthonormal basis, all other eigenvectors are orthogonal to $\mathrm{g}$ and have eigenvalues equal to $\sigma_{w}^{2}$. Thus, the decomposition in Eq. 4.22 becomes

$$
\mathbf{S}=\left(\mathcal{P}_{g}+\sigma_{w}^{2}\right) \mathrm{gg}^{\dagger}+\sum_{i=2}^{N} \sigma_{w}^{2} \mathbf{v}_{i} \mathbf{v}_{i}^{\dagger}
$$

where $\mathbf{v}_{i}^{\dagger} \mathrm{g}=0$ for $i \neq 1$. The maximum eigenvalue and eigenvector completely describe the signal subspace. 
MUSIC uses a general form of the eigenvector decomposition in which $\mathrm{V}_{s}$ is the matrix of signal space vectors and $\mathrm{V}_{n}$ is the matrix of noise eigenvectors. Assuming $N_{s}$ signals in white noise, the vectors in $\mathrm{V}_{n}$ correspond to the $N-N_{s}$ smallest eigenvalues which are equal to the white noise power $\sigma_{w}^{2}$. If the noise is correlated, then the MUSIC algorithm typically includes a whitening transformation before computing the covariance. The orthogonality of the signal and noise subspaces lead to the following definition of the MUSIC pseudospectrum,

$$
\tilde{\mathcal{P}}_{\text {music }}=\frac{1}{\mathrm{~g}^{\dagger} \mathrm{V}_{n}^{\dagger} \mathrm{V}_{n} \mathrm{~g}} .
$$

Whenever the steering vector $\mathrm{g}$ is equal to a signal contained in $\mathrm{V}_{s}$, the denominator of Equation 4.25 goes to zero, yielding a (theoretically infinite) peak in the spectrum. $\tilde{\mathcal{P}}_{\text {music }}$ is a "pseudospectrum" because it contains no information about the signal power. Once the steering vectors associated with the signals have been identified, the powers can be estimated from the corresponding signal eigenvalues.

In order to determine the signal and noise subspaces, MIUSIC requires knowledge of the number of signals incident on the array. If this information is not available, it must be estimated from the data. Numerous papers in the literature address this problem $[43,44]$.

The following discussion derives a MUSIC formulation of the Ferrara/Parks modal estimator. As in Section 4.1, the replica vector for a coherent modal signal is

$$
\mathrm{g}=\mathrm{Ea}
$$

where $\mathrm{a}$ is a unit length vector. The resulting MUSIC pseudospectrum is

$$
\tilde{\mathcal{P}}_{\text {music }}=\frac{1}{\mathrm{a}^{\dagger} \mathrm{E}^{\dagger} \mathrm{V}_{n} \mathrm{~V}_{n}^{\dagger} \mathrm{Ea}}
$$

The signal and noise subspaces are determined from the eigenvalue problem shown below

$$
\mathrm{Sv}=\lambda \mathrm{v}
$$

The convention used in this thesis is to number the eigenvalues in descending order, i.e., $\lambda_{1} \geq \lambda_{2} \cdots \geq \lambda_{N}$. Since the modal signal is coherent, the signal subspace contains only one (un-normalized) eigenvector Ea that is associated with the maximum eigenvalue $\lambda_{1}$. For 
one modal signal against a background of white noise, the signal space eigenvalue is shown below

$$
\lambda_{1}=\mathcal{P}_{\mathrm{T}}\left(\mathrm{a}^{\dagger} \mathrm{E}^{\dagger} \mathrm{Ea}\right)+\sigma_{w}^{2}
$$

In this case $\mathrm{V}_{n}$ is an $N \times(N-1)$ matrix, and the resulting MUSIC pseudospectrum is

$$
\tilde{\mathcal{P}}_{\text {music }}=\frac{1}{\mathrm{a}^{\dagger} \mathrm{E}^{\dagger} \mathrm{V}_{n} \mathrm{~V}_{n}^{\dagger} \mathrm{Ea}}
$$

Applying the Ferrara/Parks method to the MUSIC algorithm leads to the following constrained optimization problem

$$
\widehat{\mathbf{a}}_{\text {music }}=\max _{\mathbf{a}}\left\{\tilde{\mathcal{P}}_{\text {music }}\right\}=\max _{\mathbf{a}}\left\{\frac{1}{\mathrm{a}^{\dagger} \mathbf{E}^{\dagger} \mathbf{V}_{n} \mathbf{V}_{n}^{\dagger} \mathrm{Ea}}\right\} \text { subject to } \mathrm{a}^{\dagger} \mathbf{a}=1
$$

The purpose of the constraint is to eliminate the trivial solution. This optimization is basically identical to the optimization required for the MV derivation. Stationary points of the MUSIC pseudospectrum are defined by an eigenvalue equation

$$
\mathrm{E}^{\dagger} \mathrm{V}_{n} \mathrm{~V}_{n}^{\dagger} \mathrm{Ey}=\nu \mathrm{y}
$$

The strongest peak in $\tilde{\mathcal{P}}_{\text {music }}$ is associated with $\nu_{\text {min }}$, and therefore the maximizing modal distribution is $\mathrm{y}_{\min }$, i.e.,

$$
\widehat{\mathrm{a}}_{\mathrm{music}}=\mathrm{y}_{\min } \text {. }
$$

As noted above, the MUSIC pseudospectrum is not a proper power spectrum, thus $\nu_{\min }^{-1}$ does not provide an estimate of the signal power. Instead MUSIC derives the power estimate $\mathcal{P}_{\text {music }}$ from the signal space eigenvalue $\lambda_{1}$ as follows

$$
\mathcal{P}_{\text {music }}=\frac{\lambda_{1}}{\widehat{\mathrm{a}}_{\text {music }}^{\dagger} \mathrm{E}^{\dagger} \mathrm{E} \widehat{\mathrm{a}}_{\text {music }}} \text {. }
$$

Based on Equations 4.33 and 4.34, the MUSIC estimate of the mode correlation matrix is defined to be

$$
\widehat{\mathrm{S}}_{\mathrm{M} \_ \text {MUSIC }}=\left(\frac{\lambda_{1}}{\mathrm{y}_{\min }^{\dagger} \mathrm{E}^{\dagger} \mathrm{E} \mathrm{y}_{\min }}\right) \mathrm{y}_{\min } \mathrm{y}_{\min }^{\dagger}
$$

The deep water example (previously used in Sections 3.1 and 4.1) illustrates the key 
aspects of the MUSIC estimator. Sections 4.2.1-4.2.4 discuss MUSIC's performance with respect to the issues of noise, mode orthogonality, snapshots and signal coherence.

\section{Deep Water Example}

The salient parameters of the simulation environment are given in Section 4.1 (see page 51). The processing attempts to resolve the first 10 modes of the waveguide using ideal sensor covariance matrices. All plots show the least squares and minimum variance results for comparison purposes.

Figure 4-11 is a plot of the ideal MUSIC estimates of the average modal powers for three different signal-to-noise ratios: $10 \mathrm{~dB}, 0 \mathrm{~dB}$, and -10 dB. At each SNR level, the MV and MUSIC estimates for modes 1-8 are indistinguishable. Neither of the Ferrara/Parks processors exhibit the bias error that plagues the LS estimator at low signal-to-noise ratios. The key difference between the minimum variance and MUSIC algorithms is shown in the estimates of modes 9 and 10. The MUSIC estimate is effectively $0(-\infty \mathrm{dB})$ for each of these modes, while the MV estimate is in the -70 to $-50 \mathrm{~dB}$ range for the $0 \mathrm{~dB}$ and $-10 \mathrm{~dB}$ signal-to-noise ratios, respectively. Both MV and MUSIC offer significant improvements over the LS estimate of modes 9 and 10.

In order to understand the MUSIC estimator's behavior, consider the eigenvalues and eigenvectors of $\mathrm{E}^{\dagger} \mathrm{V}_{n} \mathrm{~V}_{n}^{\dagger} \mathrm{E}$ where $\mathrm{V}_{n}$ is determined from the ideal sensor covariance matrix. Recall that the received signal for the deep water example includes a coherent modal signal plus white noise. Equation 4.9 gives the ideal sensor covariance for this example. Because of the orthogonality of the subspaces, $\mathrm{V}_{n} \mathrm{~V}_{n}^{\dagger}$ can be written as follows

$$
\mathrm{V}_{n} \mathrm{~V}_{n}^{\dagger}=\mathrm{I}-\mathrm{V}_{s} \mathrm{~V}_{s}^{\dagger}=\mathrm{I}-\frac{\left(\mathrm{Ea}_{\mathrm{T}}\right)\left(\mathrm{Ea}_{\mathrm{T}}\right)^{\dagger}}{\left\|\mathrm{Ea}_{\mathrm{T}}\right\|^{2}}
$$

where the $\left\|\mathrm{Ea}_{\mathrm{T}}\right\|$ term is included to ensure that the signal eigenvector is properly normalized. Using Equation 4.36 the eigenvalue problem for MUSIC is

$$
\left[\mathrm{E}^{\dagger} \mathrm{E}-\left(\frac{1}{\mathrm{a}_{\mathrm{T}}^{\dagger} \mathrm{E}^{\dagger} \mathrm{E} \mathrm{a}_{\mathrm{T}}}\right) \mathrm{E}^{\dagger} \mathrm{E} \mathrm{a}_{\mathrm{T}} \mathrm{a}_{\mathrm{T}}^{\dagger} \mathrm{E}^{\dagger} \mathrm{E}\right] \mathrm{y}=\nu \mathrm{y} .
$$

The assumption of orthogonal sampled modeshapes permits an analytical solution of this 

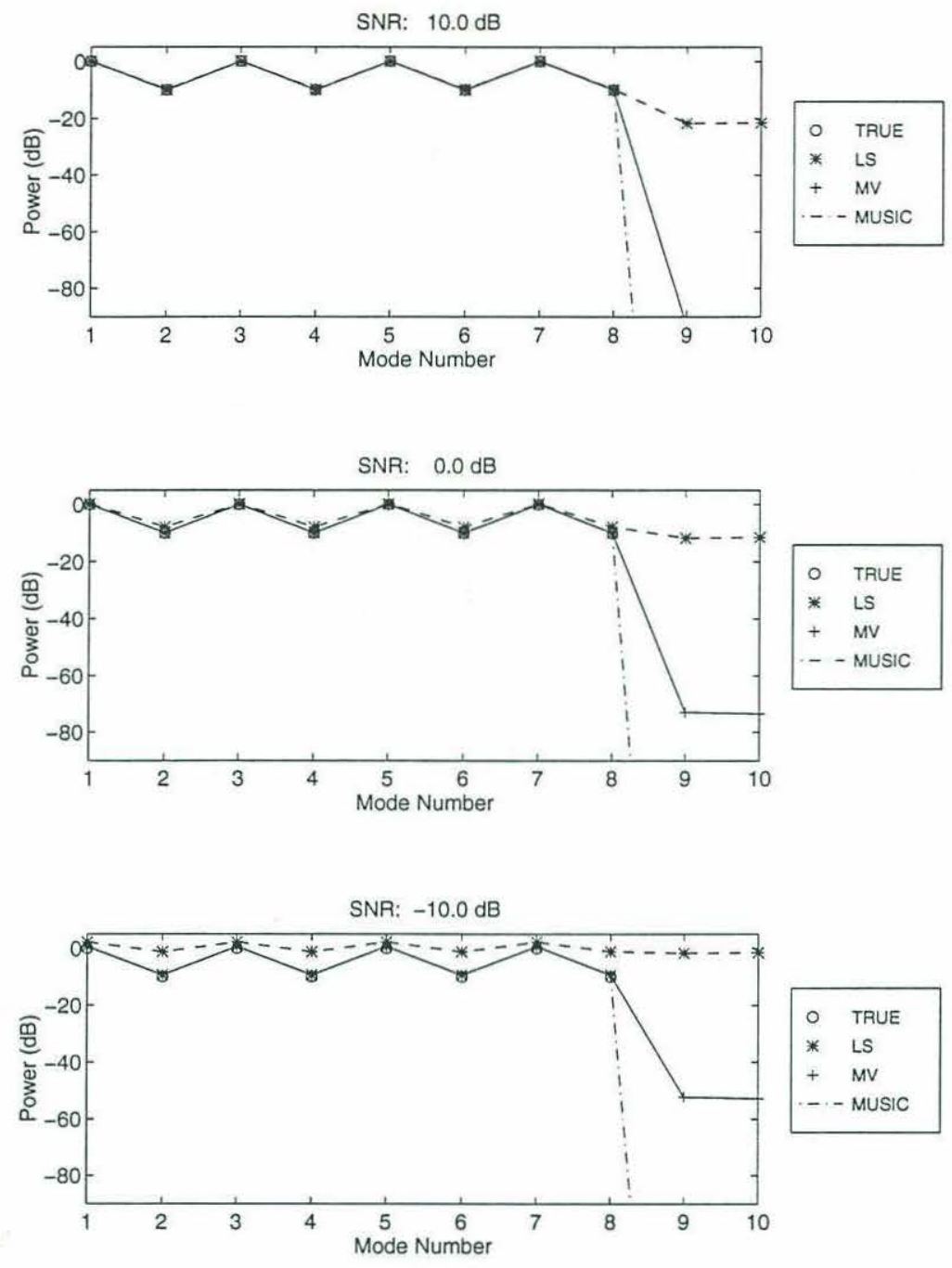

Figure 4-11: MUSIC power estimates for the deep water example

eigenvalue problem. Assuming that $\mathbf{E}^{\dagger} \mathbf{E}=\beta \mathbf{I}$, Equation 4.37 becomes

$$
\beta\left[\mathbf{I}-\mathbf{a}_{\mathrm{T}} \mathbf{a}_{\mathrm{T}}^{\dagger}\right] \mathbf{y}=\nu \mathbf{y}
$$

From this equation, it is trivial to show that $\mathrm{a}_{\mathrm{T}}$ is an eigenvector of $\mathrm{E}^{\dagger} \mathrm{V}_{n} \mathrm{~V}_{n}^{\dagger} \mathrm{E}$ corresponding to a zero eigenvalue. All the other eigenvalues are equal to $\beta$. Thus $\mathrm{a}_{\mathrm{T}}$ is the minimum eigenvector. These calculations also illustrate the point that $\tilde{\mathcal{P}}_{\text {music }}$ is not a proper power spectrum since $\nu_{\min }^{-1}$ is clearly infinite. Based on Equation 4.34, the MUSIC estimates for 
the power and relative distribution in the ideal case are shown below:

$$
\mathcal{P}_{\text {music }}=\mathcal{P}_{\mathrm{T}}+\frac{\sigma_{w}^{2}}{\mathrm{a}_{\mathrm{T}}^{\dagger} \mathrm{E}^{\dagger} \mathrm{Ea} \mathrm{a}_{\mathrm{T}}}=\mathcal{P}_{\mathrm{T}}+\frac{\sigma_{w}^{2}}{\beta}
$$

and

$$
\mathrm{a}_{\text {music }}=\mathrm{y}_{\min }=\mathrm{a}_{\mathrm{T}} \text {. }
$$

The MUSIC power estimate is biased by the same amount as the MV estimate. As discussed in Section 4.1, the bias can be subtracted off in the ideal case, but this correction is usually unrealistic in practice. Biased power estimates are used throughout the rest of this section.

According to the derivation above, the MUSIC estimate of the mode correlation in the ideal covariance case is identical to the MV estimate

$$
\mathrm{S}_{\mathrm{M} \_ \text {MUSIC }}=\left(\mathcal{P}_{\mathrm{T}}+\frac{\sigma_{w}^{2}}{\beta}\right) \mathrm{a}_{\mathrm{T}} \mathbf{a}_{\mathrm{T}}^{\dagger}=\mathrm{S}_{\mathrm{M}}+\frac{\sigma_{w}^{2}}{\beta} \mathbf{a}_{\mathrm{T}} \mathbf{a}_{\mathrm{T}}^{\dagger} .
$$

The plot in Figure 4-11 seems to support this conclusion, especially for modes 1-8.

A plot of the error vector $\left(10 \log _{10} \mathbf{e}_{j}\right)$ is shown in Figure 4-12 for the three SNR levels used in the deep water example. At $10 \mathrm{~dB}$ SNR, the MUSIC error curve is approximately $20 \mathrm{~dB}$ lower than the MV curve, but both are consistently lower than the LS error. For $0 \mathrm{~dB}$ and $-10 \mathrm{~dB}$ SNR the MV and MUSIC errors for modes 1-8 are identical. MUSIC always has less error in the null modes (9 and 10) than the minimum variance formulation. Although according to Equations 4.16 and 4.41 the two Ferrara/Parks estimators are identical, the error plots indicate several differences. To understand these differences, recall that the simplifying assumption of orthogonal modes was used to derive the expressions for the ideal covariance results, $\mathbf{S}_{\mathrm{M} \_\mathrm{MV}}$ and $\mathbf{S}_{\mathrm{M} \_ \text {MUSIC. }}$. For the deep water example, $\mathbf{E}^{\dagger} \mathrm{E}$ is only approximately a scaled identity matrix. The differences in the MV and MUSIC estimates can be attributed to the ways in which each processor handles perturbations from the perfectly orthogonal mode structure. From this example MUSIC appears to be slightly more robust with respect to the orthogonality of the sampled modeshapes.

Based on the results described above, MUSIC appears to perform better than the minimum variance method in certain situations. The rest of this section contains the performance analysis of the MUSIC estimator with respect to the issues of ambient noise, modal orthogonality, estimated covariances, and signal coherence. Each subsection draws appro- 

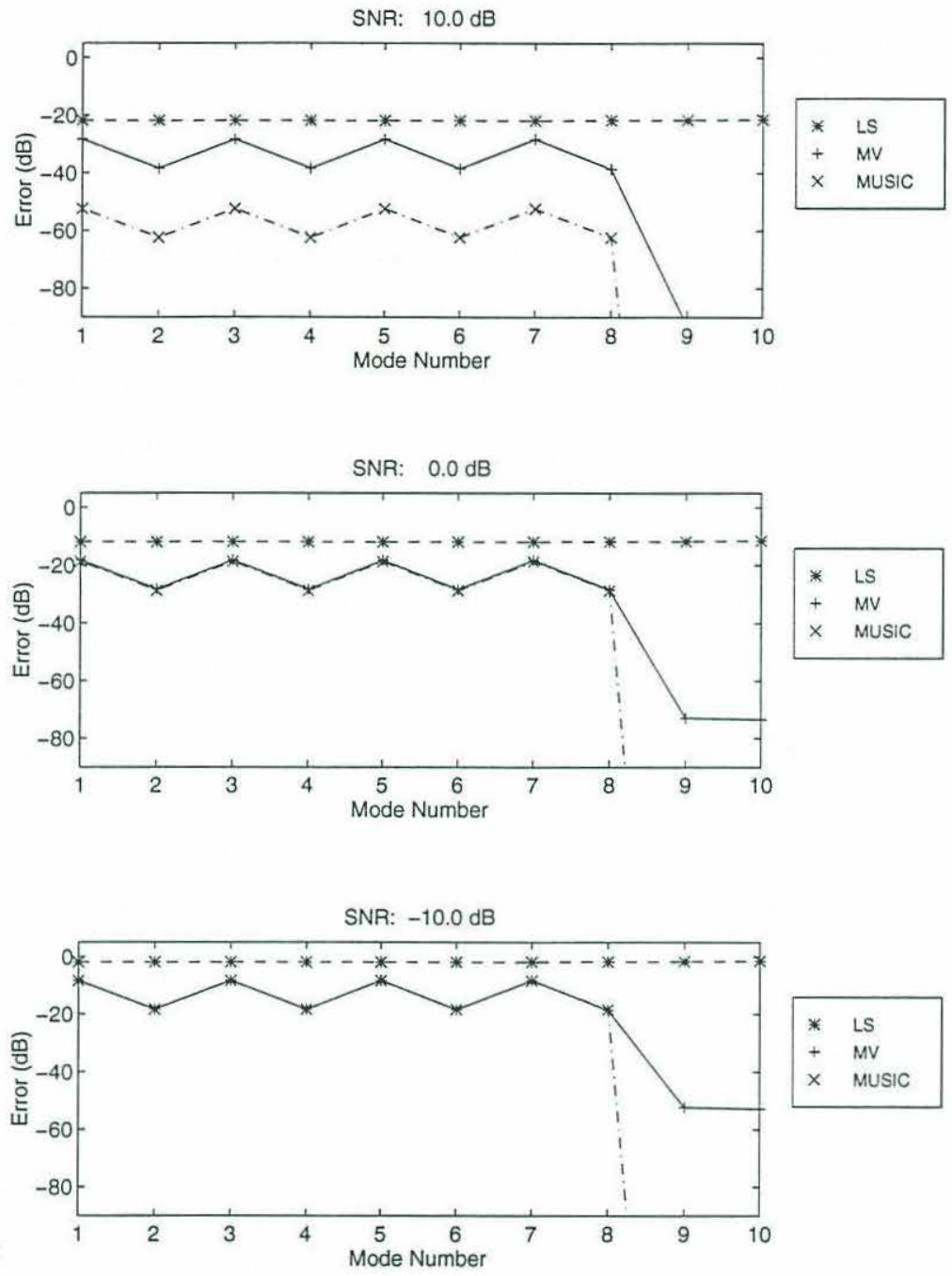

Figure 4-12: Error in MUSIC power estimates for the deep water example

priate comparisons between both Ferrara/Parks formulations and the conventional least squares formulation.

\subsubsection{Ambient Noise}

Section 4.1.1 indicates that the Ferrara/Parks minimum variance formulation performs much better than least squares methods with respect to ambient noise conditions. Since the MUSIC algorithm uses a subspace decomposition to reduce the effects of noise on the power estimates, it is expected to have similar advantages. The purpose of this section is to examine error as a function of SNR for the MUSIC formulation. 
As shown above, the MV and MUSIC estimates are identical in the ideal covariance case, provided that the sampled modeshapes are orthogonal. As a result, the MUSIC error is given by Equation 4.17; this implies that the error for each mode is a function of the noise power and the relative amplitude.

It is useful to revisit the example given in Section 4.1.1. The parameters are the same as the deep water example given above, with the exception that the noise power is varied so that the SNR varies from $-20 \mathrm{~dB}$ to $20 \mathrm{~dB}$. Figure $4-13$ shows a plot of error vs. SNR for modes 1, 2, and 10. For low SNR's the MV and MUSIC errors are almost identical, lending
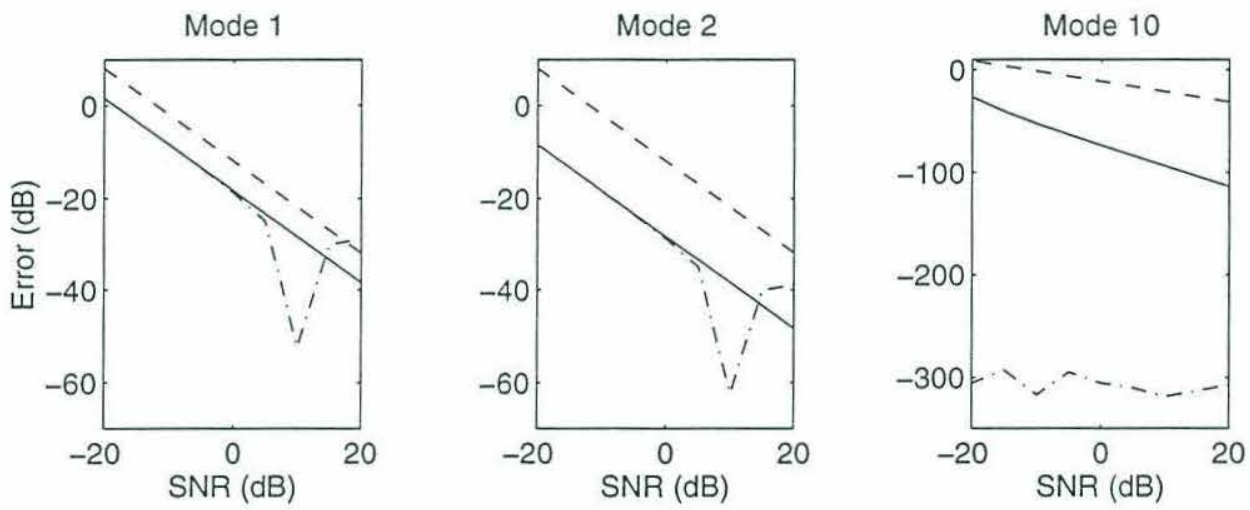

$$
-\cdots \text { LS }
$$$$
\text { MV }
$$$$
\text { -. MUSIC }
$$

Figure 4-13: Error vs. SNR for modes 1, 2, and 10 of the deep water waveguide. Ambient noise consists of white sensor noise only. MUSIC, MV, and LS error curves are shown for the ideal covariance case. Note that the vertical scale for mode 10 differs from the one used for modes 1 and 2 .

some credibility to the model derived above. The key difference in the MV and MUSIC estimates is for mode 10 . Recall that mode 10 is not present in the test signal, thus the error should be zero according to Eq. 4.17. While the error for the MUSIC estimator agrees with this prediction, the MV error does not. The discussion in Section 4.1 .1 attributes the non-zero MV error to the fact that the sampled modes are not perfectly orthogonal. Again, this is an indication that MUSIC is more robust than minimum variance with respect to the orthogonality of the sampled modeshapes. Note that the MUSIC error curves for modes 1 and 2 dip sharply at $10 \mathrm{~dB}$ and then appears to level off. The simplified model of the error, derived assuming orthogonal modes, cannot account for this anomaly. If the MUSIC formulation is used in a high SNR environment, further research is necessary in order to 
explain this behavior.

\subsubsection{Modal Orthogonality}

From the preceding examples it appears that the MUSIC estimator is more tolerant of nonorthogonal modeshapes than the MV or LS algorithms. This section addresses the modal orthogonality issue directly using the two examples developed for Sections 3.1.2 and 4.1.2.

Suppose that it is necessary to estimate the power in the first 16 modes of the deep water waveguide using the 40-element array. In this case the sampled modeshapes are not orthogonal $\left(D O F_{\text {eff }}=14\right)$. Assume an input signal consisting of all 16 modes: the odd modes are excited at $0 \mathrm{~dB}$ and the even modes are excited at $-10 \mathrm{~dB}$. The average SNR is fixed at $0 \mathrm{~dB}$, and the ideal sensor covariance is used to generate the estimates. Figure 414 shows the 16 -mode error vector $\left(10 \log _{10} \mathbf{e}_{j}\right)$ for the MUSIC, MV, and LS algorithms. Although LS and MV suffer large errors for the highest order modes, the MUSIC error

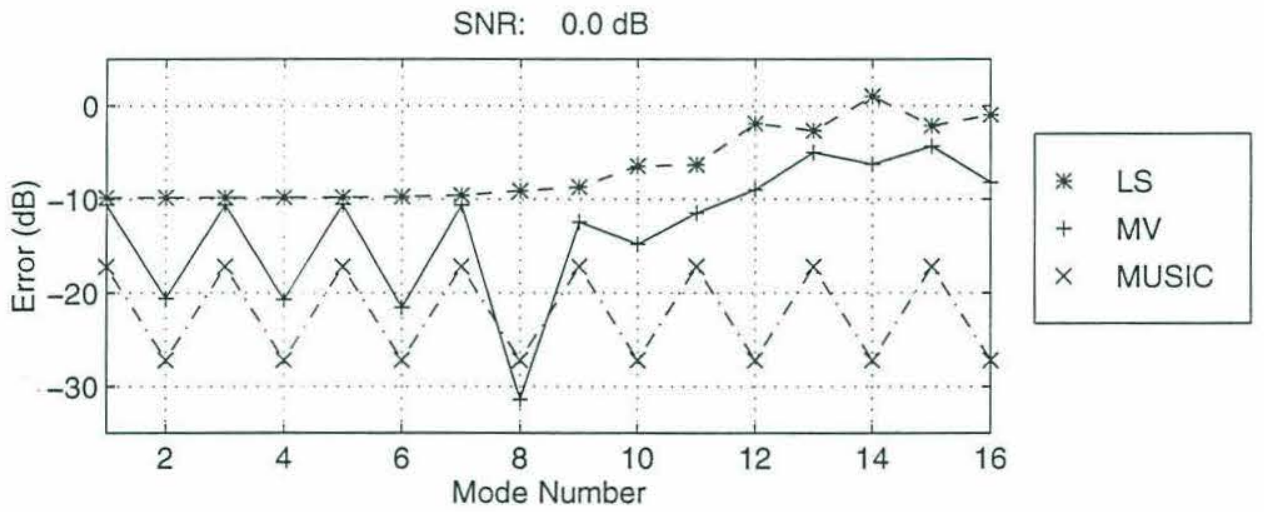

Figure 4-14: Error vector for the 16-mode example using the deep water waveguide. MUSIC, $\mathrm{MV}$, and LS results are shown.

follows the expected alternating pattern for modes 1 through 16 . The loss of two degrees of freedom in $\mathrm{E}^{\dagger} \mathrm{E}$ appears to have little effect on the Ferrara/Parks MUSIC formulation.

Consider a second example. The parameters are identical to the first deep water example in this section, but the number of modes to estimate is varied from 8 up to 16. Recall that the test signal consists of the first 8 modes in an alternating $0 \mathrm{~dB} /-10 \mathrm{~dB}$ pattern. The simulation uses ideal sensor covariance matrices. Figure 4-15 is a plot of the total error vs. number of modes to estimate for two different input SNR's: $0 \mathrm{~dB}$ and $-10 \mathrm{~dB} .{ }^{4} \mathrm{At} 0 \mathrm{~dB}$

\footnotetext{
${ }^{4}$ Recall that the total error is defined as $\operatorname{TR}\left\{\left|\widehat{S}_{M_{-} \text {MUSIC }}-\mathrm{S}_{M}\right|\right\}$.
} 

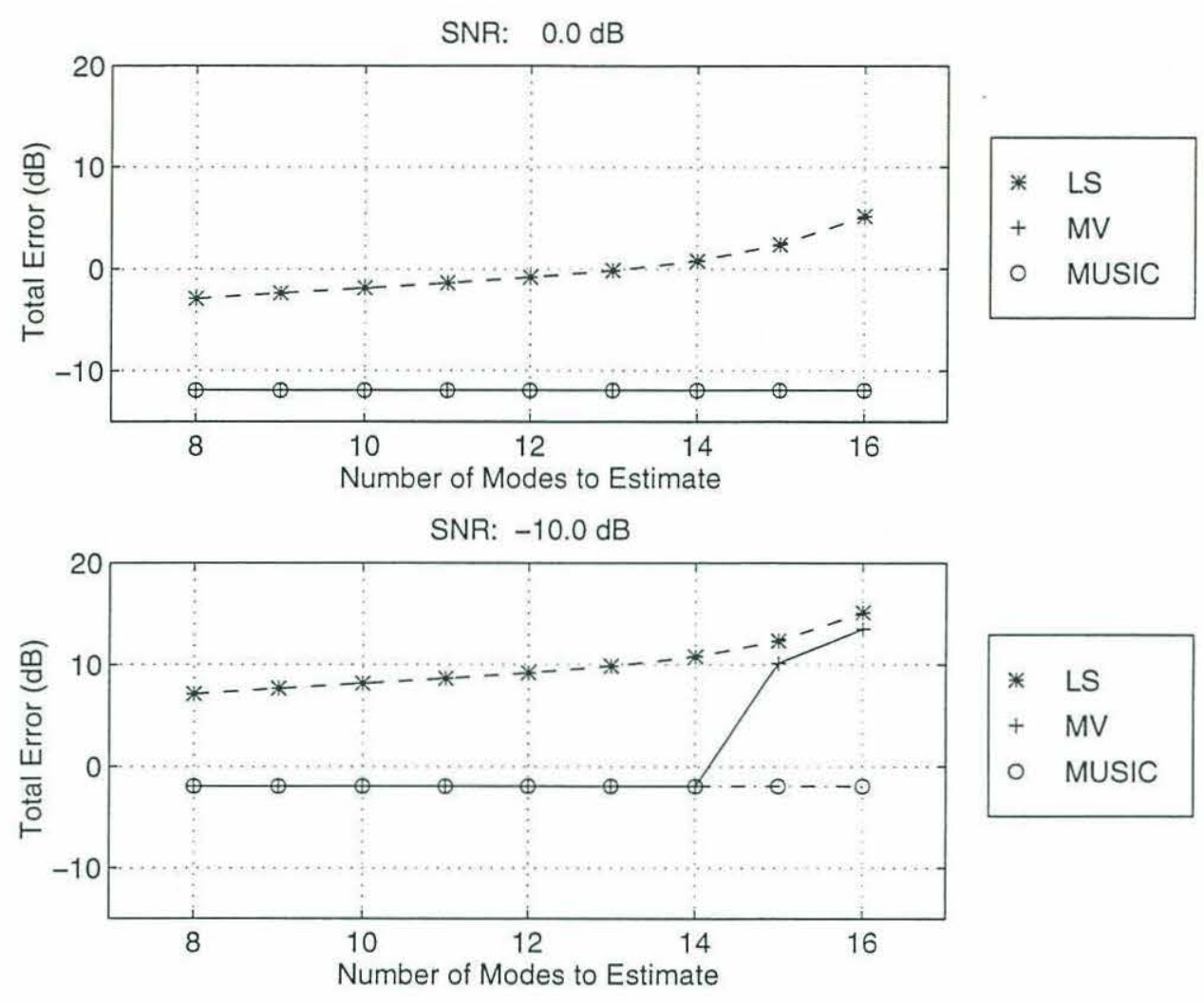

Figure 4-15: Total error vs. number of modes to estimate for the deep water example. Results for two input SNR's $(0 \mathrm{~dB}$ and $-10 \mathrm{~dB})$ are shown.

SNR the results for both of the Ferrara/Parks formulations are identical. The errors are constant regardless of the number of modes included in the estimate (up to mode 16). The $-10 \mathrm{~dB}$ SNR case differentiates the MV and MUSIC estimates. For 15 and 16 modes in the estimate, the minimum variance method has extreme difficulty and displays error levels close to that of the least squares method. On the other hand, MUSIC maintains a constant error for the 15 and 16 mode cases.

These two examples illustrate a favorable characteristic of the MUSIC modal estimator, namely robustness with respect to orthogonality of the sampled modes. This property of the estimate offers advantages since practical arrays often do not guarantee the orthogonality of the desired subset of modes.

\subsubsection{Estimated Covariance Issues}

It is very important to consider the effects of using estimated instead of ideal covariances on adaptive estimators such as MUSIC. Appendix D derives the mean and variance associated 
with the MUSIC power estimates using known characteristics of the sample covariance matrix $\widehat{\mathrm{S}}$.

A simplifying assumption is required in order to derive an expression for the first and second order statistics of the estimates. Specifically, the following discussion assumes that MUSIC can exactly determine the relative modal distribution, therefore any errors are associated solely with the signal power estimate. Under this assumption, the expected power in mode $j$ (assuming white noise only) is given below

$$
\mathcal{E}\left\{\widehat{P}_{j_{\text {_music }}}\right\} \equiv \bar{P}_{j_{\text {_music }}}=P_{j}+\frac{\sigma_{w}^{2}}{\beta}\left[\mathrm{a}_{\mathrm{T}} \mathbf{a}_{\mathrm{T}}^{\dagger}\right]_{j j} .
$$

The variance, also derived in Appendix D, is shown below

$$
\operatorname{Var}\left\{\widehat{P}_{j \text { _music }}\right\}=\frac{\left(\bar{P}_{j \text { _music }}\right)^{2}}{L} .
$$

Based on these two equations, it is possible to predict bias and variance for the MV estimates. The following example contrasts the predictions with the results of a Monte Carlo simulation. This is useful in determining the validity of the assumption used to derive the bias and variance. The parameters of the simulation are identical to the deep water example using the test signal. Each data point is the result of averaging over 1000 independent trials.

Figure 4-16 is a plot of the error vs. the number of snapshots for mode 1 . The predicted and Monte Carlo errors are shown for all three estimators: MUSIC, MV, and LS. MUSIC has lower errors than either of the other two estimates for any number of snapshots. In addition, the MUSIC results agree with the predictions.

Figure 4-17 shows the variance vs. number of snapshots. The predicted and Monte Carlo values are shown, along with the Cramer Rao bound. According to Appendix A, the C-R bound is a function of $\frac{1}{L}$, and Eq. 4.43 indicates that the MUSIC estimate has the same dependence on $L$. Note that the figure shows that the predicted variance is actually below the $\mathrm{C}-\mathrm{R}$ bound. This is probably due to the fact that the prediction only includes the effects of the MUSIC power estimate and not the eigenvector estimate. 


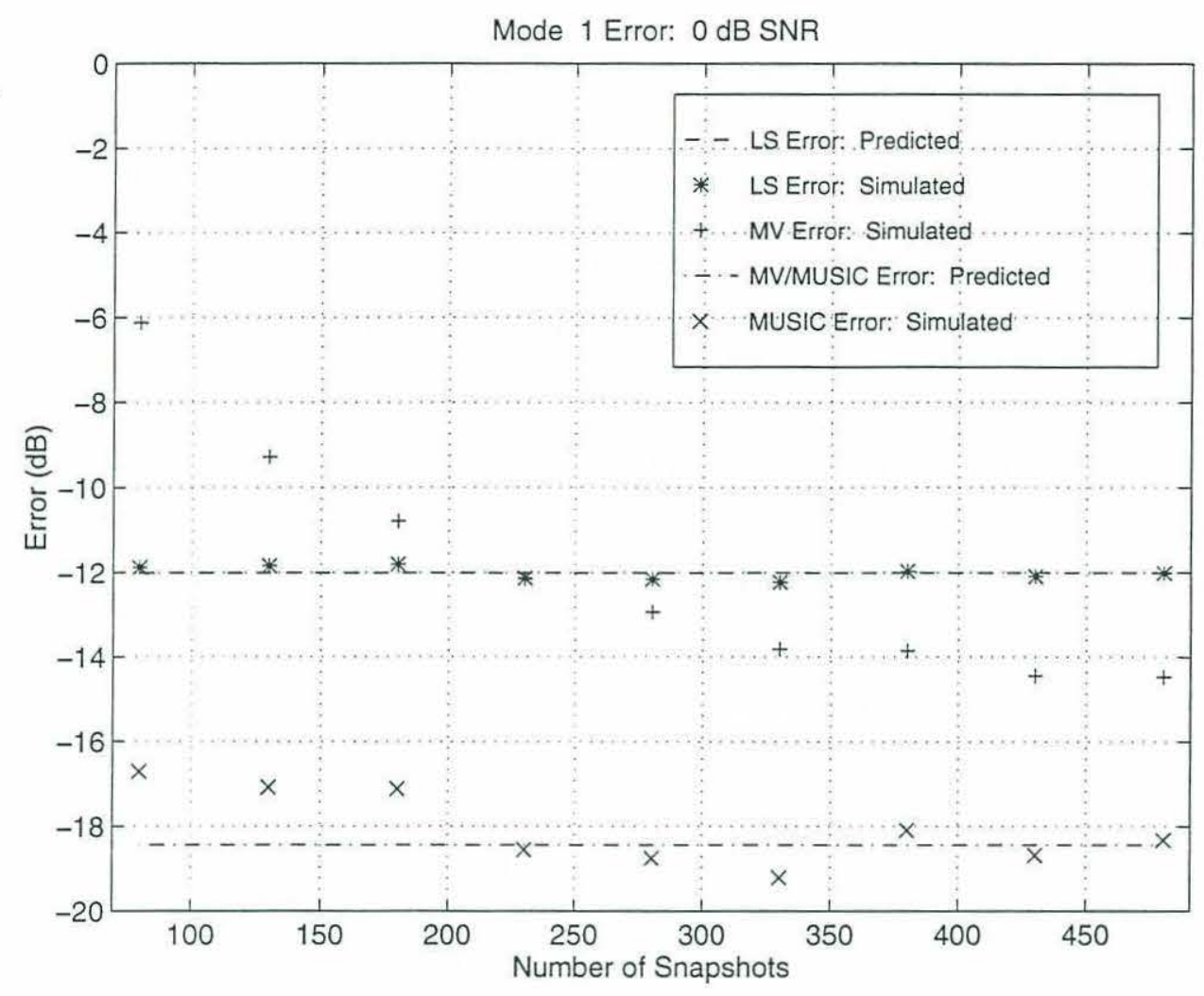

Figure 4-16: Error vs. number of snapshots for mode 1. Predicted and Monte Carlo results are shown for all three estimators.

\subsubsection{Signal Coherence}

The Ferrara/Parks approach outlined in this chapter relies on a coherent signal model. As indicated in Section 4.1.4, the MV processor has more severe estimation errors than the least squares processor if the signal is not coherent. Similar results are expected for the MUSIC formulation.

Consider repeating the coherence example that is described in Section 4.1.4 (see page 63). Figure 4-18 shows the total error vs. coherence for the MUSIC, MV, and LS estimators. The MV and MUSIC error curves are virtually identical for this example. This is reasonable since modal orthogonality and number of snapshots are not critical issues for the example. Clearly neither of the Ferrara/Parks formulations offer any advantages over least squares for partially or totally incoherent signals. 


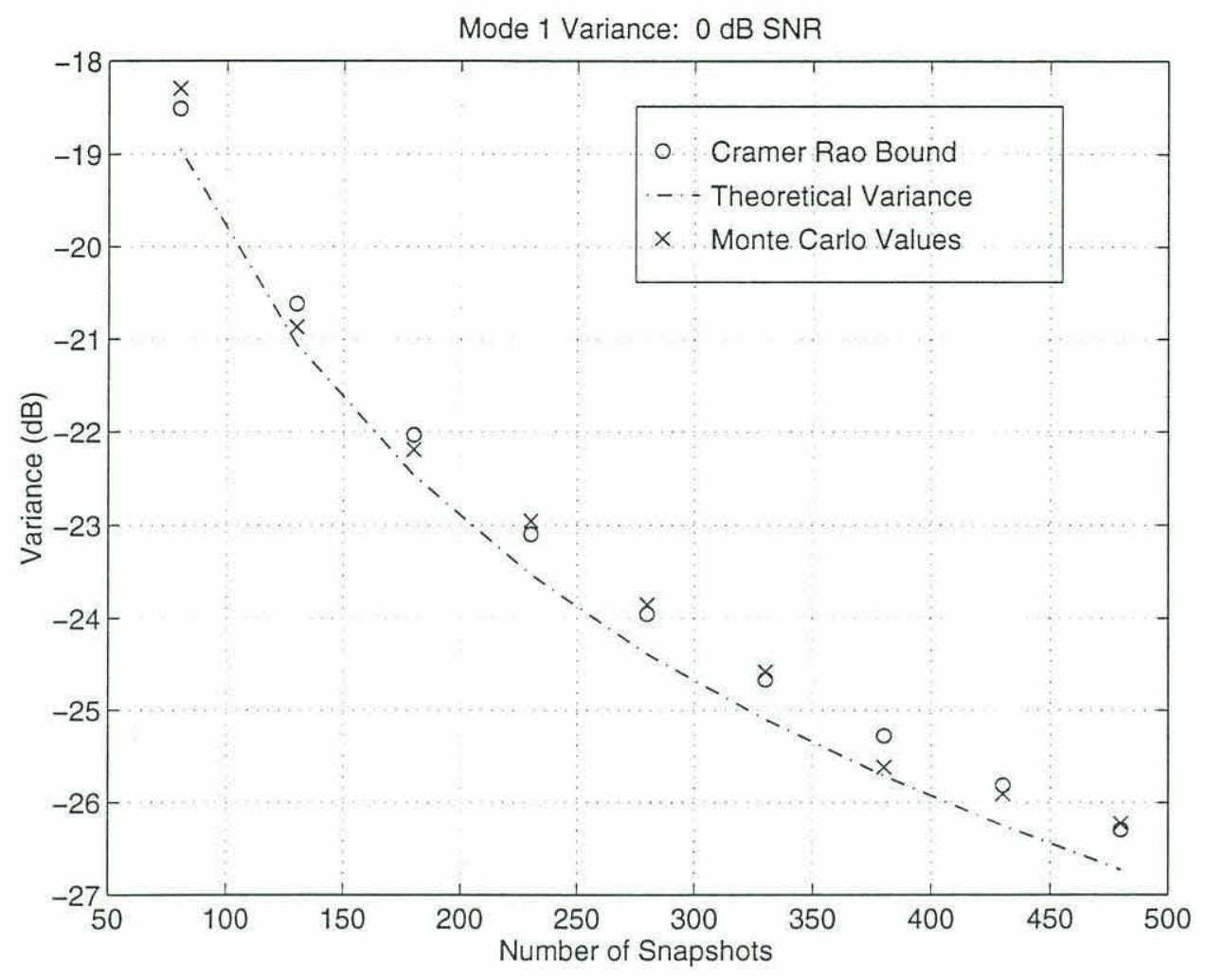

Figure 4-17: Variance vs. number of snapshots for the MUSIC estimator.

\subsection{Summary}

This chapter has developed an adaptive modal estimator based on the earlier work of Ferrara and Parks concerning array processing using diversely-polarized antenna arrays. The new approach is fundamentally different from least squares because it relies on maximizing the received signal power. Minimum variance and MUSIC formulations have been presented and analyzed. Results for both of the Ferrara/Parks methods are favorable, but it appears that MUSIC is more robust with respect to mode orthogonality. One important characteristic of both new formulations is that they have much greater potential for maintaining nulls in the modal spectrum than the conventional methods. The primary drawback of the MV and MUSIC algorithms discussed in this chapter is that they rely on a coherent signal model. As indicated in Sections 4.1.4 and 4.2.4, the performance of these estimators degrades significantly as signal coherence is lost. 


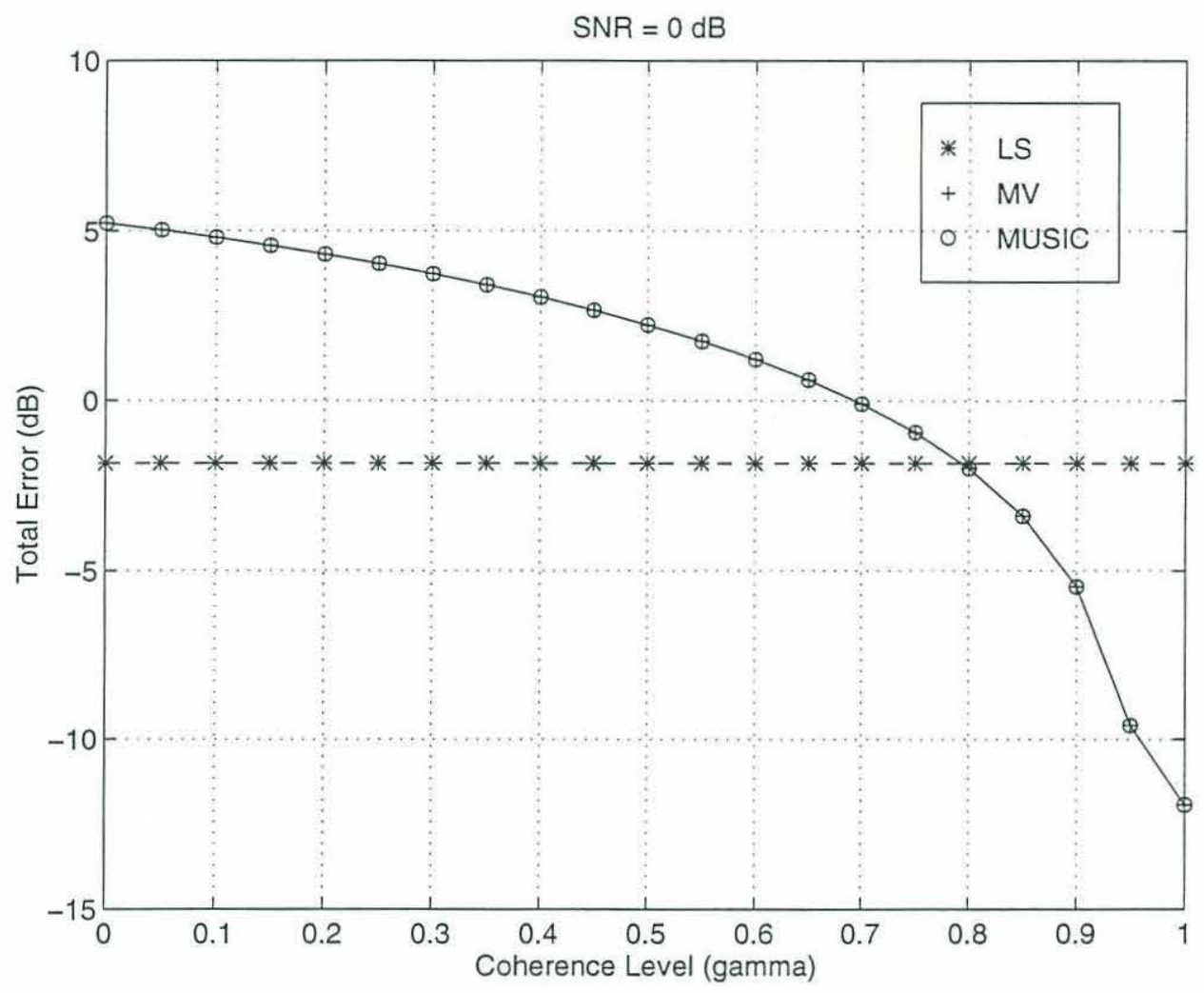

Figure 4-18: Total error vs. coherence for the Ferrara/Parks MUSIC formulation 


\section{Chapter 5}

\section{Adaptive Estimation of}

\section{Incoherent Modes}

The previous chapter has demonstrated the advantages of the Ferrara/Parks approach to the mode estimation problem. While the new method is a useful tool for coherent modes, Chapter 4 indicates that performance degrades rapidly as signal coherence is lost. This is a significant issue because coherent signals are not guaranteed at long ranges from the source; inhomogeneities in the environment can randomize the phases of the modes as they propagate. The purpose of this chapter is to propose a modification to the Ferrara/Parks method which facilitates the estimation of random modes. A thorough study of the modified approach is outside the scope of this thesis. Instead, Section 5.1 derives the modified minimum variance processor and offers a simple example to illustrate its potential advantages. The limitations of the modified Ferrara/Parks algorithm are briefly discussed. Section 5.2 summarizes and indicates several directions for further research.

\subsection{Modified Minimum Variance Formulation}

This section modifies the minimum variance processor, developed in Section 4.1, to permit the estimation of modal powers in phase-randomized signals. As a starting point, consider the eigenvector decomposition of the mode correlation matrix

$$
\mathbf{S}_{\mathrm{M}}=\sum_{i=1}^{K} \xi_{i} \mathbf{t}_{i} \mathbf{t}_{i}^{\dagger}=\mathbf{T} \Xi \mathbf{T}^{\dagger}
$$


where $K$ is the rank of $\mathrm{S}_{\mathrm{M}}$. For coherent modes $\mathrm{S}_{\mathrm{M}}$ is a rank one matrix. The total signal power $\mathcal{P}_{\mathrm{T}}$ is the eigenvalue $\xi_{1}$, and the relative modal distribution $\mathrm{a}_{\mathrm{T}}$ is the corresponding eigenvector $t_{1}$. Using a maximum power criterion, the Ferrara/Parks method produces an estimate of the modal distribution and the signal power. In other words, the method of Chapter 4 provides estimates of $t_{1}$ and $\xi_{1}$. For the general case, the mode correlation matrix has $K$ eigenvalue-eigenvector pairs which must be estimated. Since $\mathbf{S}_{\mathrm{M}}$ is a symmetric matrix, the eigenvectors form an orthogonal basis. A simple modification to the Ferrara/Parks method exploits this property in order to generate estimates of the set of $K$ eigenvectors.

As derived in Chapter 4, the eigenvalue problem stated below determines the stationary points of the minimum variance power spectrum, $\mathcal{P}_{\mathrm{mv}}$.

$$
\mathrm{E}^{\dagger} \mathrm{S}^{-1} \mathrm{Ey}_{i}=\nu_{i} \mathrm{y}_{i}
$$

Recall that the convention in this thesis is to order the eigenvalues as follows: $\nu_{1} \geq \nu_{2} \geq \cdots \geq \nu_{M}$. Given this ordering, routine matrix algebra leads to bounds on $\mathcal{P}_{\mathrm{mv}}[39]$

$$
\frac{1}{\nu_{1}} \leq \mathcal{P}_{\mathrm{mv}} \leq \frac{1}{\nu_{M}}
$$

The Ferrara/Parks estimate of the first eigenvector, $t_{1}$, corresponds to the vector that maximizes $\mathcal{P}_{\mathrm{mv}}$; the inverse of the associated eigenvalue is the maximum power. This implies that $\hat{\mathrm{t}}_{1}=\mathrm{y}_{M}$ and $\hat{\xi}_{1}=\nu_{M}^{-1}$. Based on the orthogonality property mentioned above, a reasonable choice for the second eigenvector is the vector which maximizes $\mathcal{P}_{\text {mv }}$ subject to the constraint that it is orthogonal to $\hat{t}_{1}$. Since the eigenvectors defined by Equation 5.2 form an orthonormal set, the vector which maximizes $\mathcal{P}_{\mathrm{mv}}$ and is orthogonal to $\hat{\mathrm{t}}_{1}$ is $\mathrm{y}_{M-1}$. Thus, $\hat{\mathrm{t}}_{2}=\mathrm{y}_{M-1}$ and $\hat{\xi}_{2}=\nu_{M-1}^{-1}$. A simple extension of this argument leads to estimates for the remaining eigenvectors and eigenvalues. Thus, the modified Ferrara/Parks MV estimate of the mode correlation matrix is shown below

$$
\widehat{\mathrm{S}}_{\mathrm{M} \_ \text {MV_mod }}=\sum_{i=M-K+1}^{M} \nu_{i}^{-1} \mathrm{y}_{i} \mathrm{y}_{i}^{\dagger} .
$$

Note that the derivation assumes that the rank of $\mathbf{S}_{\mathrm{M}}$ is known. In practice $K$ is not usually known and must be estimated from the data. Estimation of the rank is probably the most challenging aspect of the modified processing scheme. One approach is to set $\widehat{K}$ 
equal to the number of "significant" peaks in the power spectrum. A reasonable measure of the number of peaks is the effective degrees of freedom associated with the eigenvalue problem in Equation 5.2. Since the largest peaks correspond to the smallest eigenvalues, the $D O F_{\text {eff }}$ is computed using the inverse of the eigenvalues, i.e.,

$$
\widehat{K}=\left\lceil\frac{\left(\sum_{i=1}^{M} \frac{1}{\nu_{i}}\right)^{2}}{\sum_{i=1}^{M}\left(\frac{1}{\nu_{i}}\right)^{2}}\right\rceil .
$$

The $\lceil\cdot\rceil$ operator rounds the enclosed quantity up to the nearest integer. The estimate for $K$ given in Equation 5.5 is used in all of the examples in this chapter. Section 5.2 suggests several alternate methods for estimating the rank of $\mathbf{S}_{\mathrm{M}}$.

A simple example illustrates the basic characteristics of the modified MV processor.

\section{Deep Water Example: Partially Incoherent Modes}

The signal model is based on the adiabatic channel with internal waves described in Chapter 2. The form of the mode correlation matrix is shown below

$$
\mathbf{S}_{\mathrm{M}}=(1-\gamma) \operatorname{diag}\left[\mathbf{x}_{0} \mathbf{x}_{0}^{\dagger}\right]+\gamma \mathbf{x}_{0} \mathbf{x}_{0}^{\dagger}
$$

Recall that the vector $\mathrm{x}_{0}$ contains the deterministic mode amplitudes and $\gamma$ is the coherence parameter. For $\gamma=0$ the signal consists of a totally incoherent sum of modes and for $\gamma=1$ the sum is perfectly coherent.

The simulation environment used for the example is the deep water waveguide. The signal consists of 8 modes: the odd modes $(1,3,5,7)$ are excited at a reference level of $0 \mathrm{~dB}$ and the even modes $(2,4,6,8)$ are excited at a level of $-10 \mathrm{~dB}$. The coherence level $\gamma$ is equal to 0.5. Additive noise in the environment consists of white sensor noise only. The noise power is set so that the input SNR (geometric mean of the SNR's at each sensor) is equal to $0 \mathrm{~dB}$. The 40-element simulation array (described in Chapter 2) samples the pressure field. The processing attempts to resolve the first 10 modes of the waveguide. Ideal covariance matrices are used to generate this example.

Figure 5-1 shows the error in the modified MV power estimates for the deep water example; errors for the LS processor are shown for comparison purposes. Recall that the least squares method does not rely on a coherent signal model, therefore no modifications 
to the LS processor are necessary. The error vector e is defined (see Equation 2.29) as the

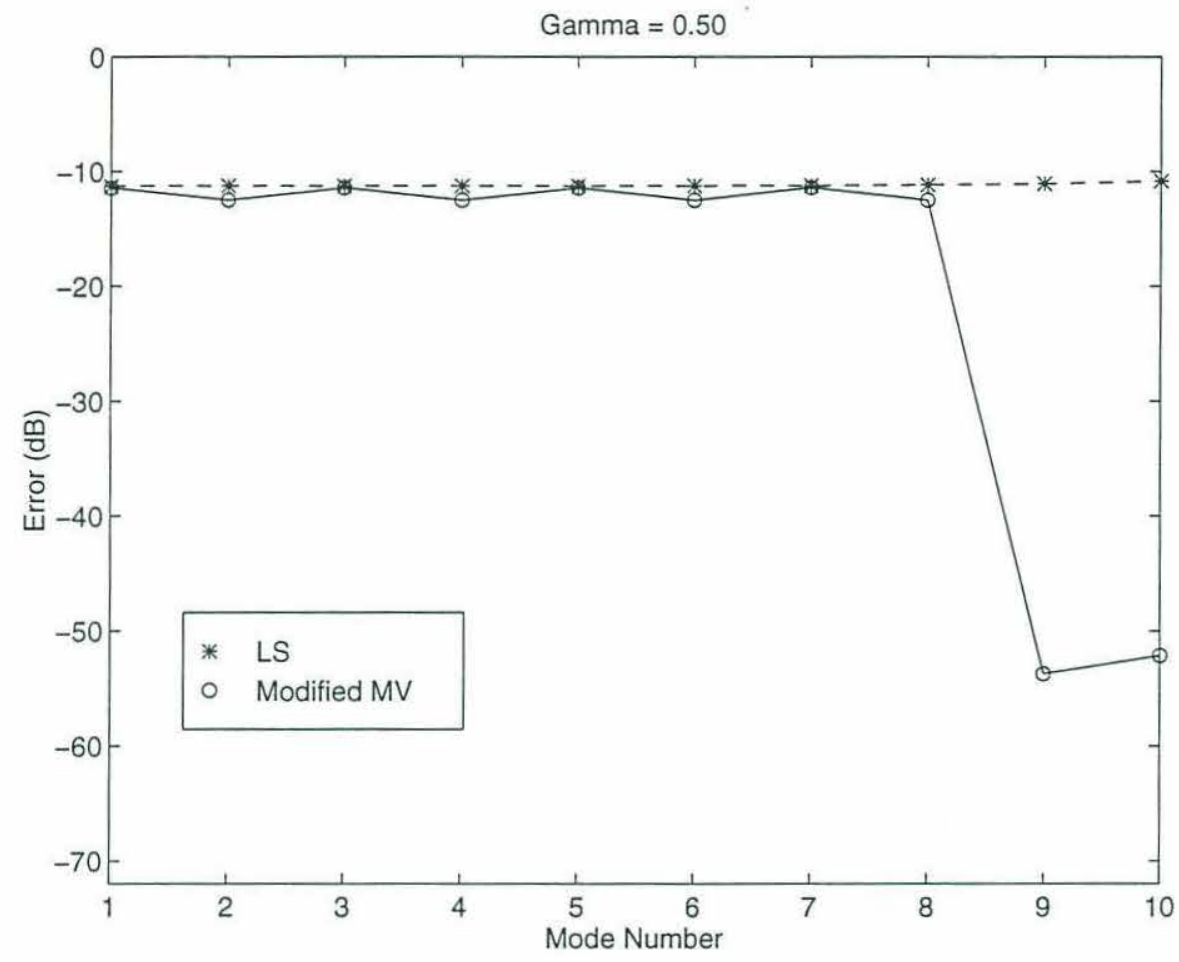

Figure 5-1: Estimation Error for Partially Incoherent Mode Example

absolute value of the differences in the true and estimated powers. The plot displays the error on a $\log$ scale $\left(10 \log _{10} \mathbf{e}_{j}\right)$. For the modes that are present in the signal (i.e., 1-8), the modified MV algorithm incurs approximately the same error as the LS processor. For modes 9 and 10 (not contained in the signal), the extended Ferrara/Parks formulation has much smaller errors than the least squares method. As noted in Chapter 3, LS has difficulty maintaining nulls in the spectrum due to bias error. The modified MV formulation does not appear to have this problem.

In order to understand the behavior of the modified minimum variance estimator, it is useful to derive analytical expressions for the eigenvalues and eigenvectors of $\mathrm{E}^{\dagger} \mathrm{S}^{-1} \mathrm{E}$. For an incoherent signal plus additive white noise, the ideal sensor covariance is shown below

$$
\mathrm{S}=\mathrm{E}\left[\mathrm{T} \Xi \mathrm{T}^{\dagger}\right] \mathrm{E}^{\dagger}+\sigma_{w}^{2} \mathrm{I}
$$

Application of the Woodbury identity to Equation 5.7 yields the following expression for 
the inverse of $\mathbf{S}$

$$
\mathbf{S}^{-1}=\frac{1}{\sigma_{w}^{2}}\left[\mathbf{I}-\mathbf{E T D T}^{\dagger} \mathbf{E}^{\dagger}\right]
$$

where $\mathrm{D}$ is a diagonal matrix

$$
\mathrm{D}=\left[\begin{array}{ccc}
\frac{\xi_{1}}{\beta \xi_{1}+\sigma_{w}^{2}} & & \\
& \ddots & \\
& & \frac{\xi_{M}}{\beta \xi_{M}+\sigma_{w}^{2}}
\end{array}\right]
$$

Using Equation 5.8, the matrix $\mathrm{E}^{\dagger} \mathrm{S}^{-1} \mathrm{E}$ becomes

$$
\mathrm{E}^{\dagger} \mathrm{S}^{-1} \mathrm{E}=\frac{1}{\sigma_{w}^{2}}\left[\mathrm{E}^{\dagger} \mathrm{E}-\mathrm{E}^{\dagger} \mathrm{ETDT}^{\dagger} \mathrm{E}^{\dagger} \mathrm{E}\right]
$$

Finding an analytical form for the eigenvalues and eigenvectors of the above matrix requires a simplifying assumption identical to the one made in the analysis of the coherent MV estimator. Specifically, assume that the sampled modeshapes are orthogonal and that the modes are scaled equally, i.e., $\mathbf{E}^{\dagger} \mathbf{E}=\beta \mathbf{I}$. After some tedious matrix algebra, Equation 5.10 reduces to a form which explicitly gives the desired eigenvector decomposition

$$
\mathbf{E}^{\dagger} \mathbf{S}^{-1} \mathbf{E}=\mathrm{T}\left[\begin{array}{ccc}
\frac{1}{\xi_{1}+\frac{\sigma_{u j}^{2}}{\beta}} & & \\
& \ddots & \\
& & \frac{1}{\xi_{M}+\frac{\sigma_{u}^{2}}{\beta}}
\end{array}\right] \mathrm{T}^{\dagger}
$$

Under the aforementioned assumption, the eigenvectors of $\mathbf{E}^{\dagger} \mathbf{S}^{-1} \mathbf{E}$ correspond to the true eigenvectors of the mode correlation matrix. The eigenvalues of $\mathrm{E}^{\dagger} \mathrm{S}^{-1} \mathrm{E}$ are a function of both the eigenvalues of $\mathrm{S}_{\mathrm{M}}$ and the white noise power: $\nu_{i}=\frac{1}{\xi_{i}+\sigma_{w}^{2} / \beta}$. The Ferrara/Parks estimate shown in Equation 5.4 uses the $K$ smallest values of $\nu_{i}$ which, based on the observation above, are associated with the $K$ largest eigenvalues of $\xi_{i}$. Substituting this information into Equation 5.4 results in an expression for the modified MV estimate of $S_{M}$,

$$
\mathrm{S}_{\mathrm{M} \_M V \_ \text {mod }}=\underbrace{\sum_{i=1}^{K} \xi_{1} \mathrm{t}_{i} \mathrm{t}_{i}^{\dagger}}_{\mathbf{S}_{\mathrm{M}}}+\underbrace{\frac{\sigma_{w}^{2}}{\beta} \sum_{i=1}^{K} \mathrm{t}_{i} \mathrm{t}_{i}^{\dagger}}_{\text {noise terms }} .
$$

The above expression provides valuable insights into the behavior of the modified estimator. 
First, note that Equation 5.12 assumes that $K$ has been estimated correctly. In practice, the summation indices range from 1 to $\widehat{K}$. If the estimate of $K$ is less than the true value, then part of the signal is lost. Accurate estimation of the rank of $\mathbf{S}_{\mathrm{M}}$ is clearly essential for the success of the modified formulation. A second property of the modified estimator is that the noise terms only span the basis of the signal. This explains the processor's ability to maintain nulls in the modal spectrum.

It is useful to contrast the result in Equation 5.12 with the LS estimator. From Chapter 2 the LS estimate of $\mathbf{S}_{\mathrm{M}}$ (using the ideal sensor covariance) is shown below

$$
\mathbf{S}_{\mathrm{M} \_L S}=\mathbf{S}_{\mathrm{M}}+\sigma_{w}^{2}\left(\mathbf{E}^{\dagger} \mathbf{E}\right)^{-1}
$$

Assuming that the modeshapes are orthogonal $\left(\mathbf{E}^{\dagger} \mathbf{E}=\beta \mathbf{I}\right)$, the second term on the righthand side of Equation 5.13 is a multiple of the identity matrix. Expanding $\mathbf{I}$ in terms of the orthonormal basis defined by the eigenvectors of $\mathbf{S}_{\mathrm{M}}$ leads to the following expression for $\mathrm{S}_{\mathrm{M} \_L S}$

$$
\mathrm{S}_{\mathrm{M} \_\mathrm{S}}=\underbrace{\sum_{i=1}^{M} \xi_{i} \mathrm{t}_{i} \mathrm{t}_{i}^{\dagger}}_{\mathrm{S}_{\mathrm{M}}}+\underbrace{\frac{\sigma_{w}^{2}}{\beta} \sum_{i=1}^{M} \mathrm{t}_{i} \mathrm{t}_{i}^{\dagger}}_{\text {noise terms }}
$$

where $M$ is the number of modes to estimate. The key difference between the LS method and the modified MV method is in the number of eigenvectors to include in the summation. The least squares formulation always includes $M$ vectors in the sum, hence all of the modes are biased. If $K<M$, then the modified minimum variance method provides some reduction of the noise. Note that if $\mathrm{S}_{\mathrm{M}}$ is full rank $(K=M)$, then the least squares and modified minimum variance formulations are equivalent. Recall that this equivalence depends on one key assumption: the orthogonality of the sampled modeshapes.

Now consider the performance of the modified algorithm as the coherence level changes. The simulation environment is identical to the previous example, with the exception that $\gamma$ varies from 0 to 1 . Figure 5-2 is a plot of the total error versus $\gamma$ for the modified MV algorithm. The total error is defined (see Chapter 2) as $\operatorname{TR}\left\{\left|\widehat{\mathbf{S}}_{M}-\mathrm{S}_{M}\right|\right\}$. Error curves associated with the LS and the coherent MV formulations are displayed for the purpose of comparison. According to the plot, the total error for the modified MV algorithm is always below the constant error associated with the LS estimator, regardless of the coherence level. This is expected since the signal used in the example contains fewer modes than are being 


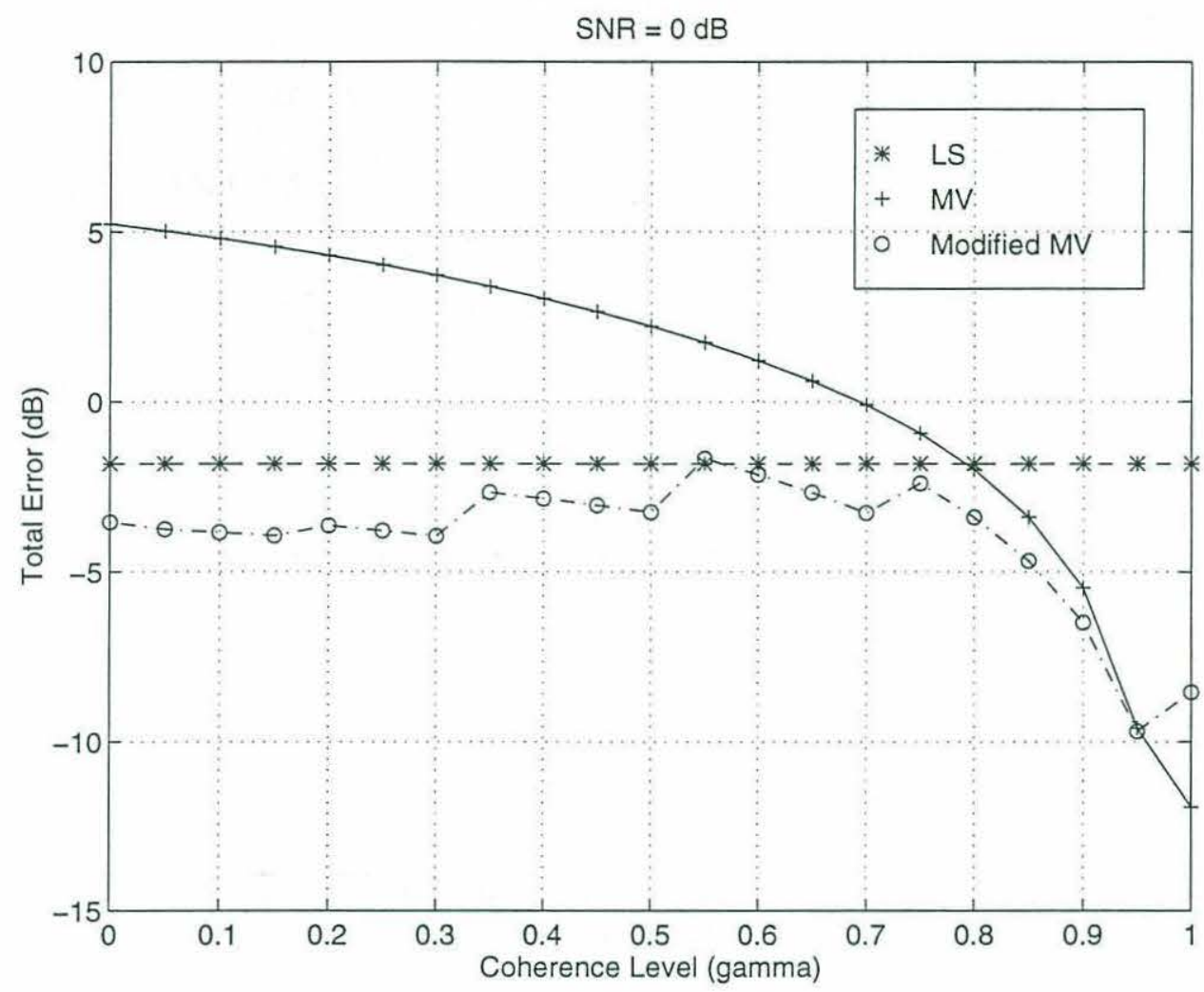

Figure 5-2: Total Error vs. Coherence for the Modified MV Algorithm. SNR=0 dB.

estimated. As derived above, the subspace decomposition used in the modified MV method offers gain over LS, provided that the true $S_{M}$ is not full rank. The coherent MV algorithm becomes useful only when $\gamma$ exceeds 0.9 . Note that the error for the modified MV algorithm is larger than the coherent MV error for $\gamma=1$. This is due to the fact that the number of vectors to include in the estimate $(K)$ is estimated incorrectly for this point. Based on this plot, the modified minimum variance formulation appears to be useful for estimating incoherent modes.

In order to avoid seeming overly optimistic about the modified algorithm, however, consider another example. The simulation environment is identical; the only difference is that the noise level is reduced so that the average SNR is now $10 \mathrm{~dB}$. A plot of the total error vs. coherence for this case is shown in Figure 5-3. As expected the modified algorithm performs better than the coherent MV formulation, but the constant error associated with the least squares processor is always below the errors for both Ferrara/Parks methods unless $\gamma$ exceeds 0.95 . This example demonstrates that for higher SNR's, the added complexity of using the modified Ferrara/Parks algorithm does not necessarily result in better estimates. 


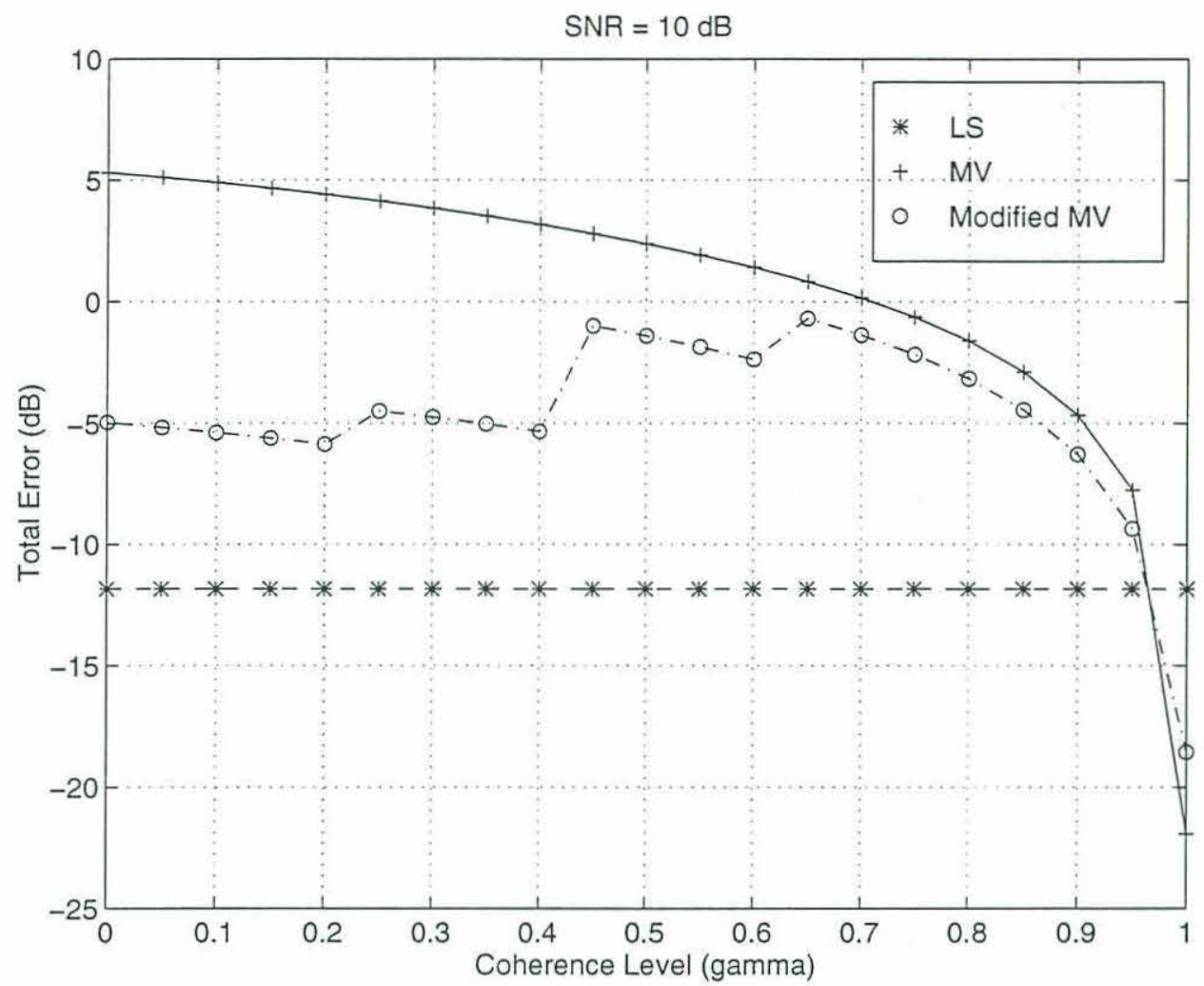

Figure 5-3: Total Error vs. Coherence for the Modified MV Algorithm. SNR=10 dB.

Further study is necessary to thoroughly evaluate the potential of the modified scheme. The next section indicates several directions for future research.

\subsection{Summary}

This chapter has outlined a method for extending the Ferrara/Parks technique to the more general problem of random mode estimation. Specifically, a modified minimum variance processor has been derived. The preliminary analysis suggests that the modified MV algorithm can reduce the effects of noise by implementing a subspace decomposition of the mode correlation matrix. A simple example has shown that the new method has some advantages over the conventional least squares formulation, but many issues remain unresolved.

Further research is necessary to assess the effects of noise and modal orthogonality on the estimates. In addition note that the examples of Section 5.1 were generated using ideal covariance matrices. The snapshot issues surrounding the estimation of the sensor covariance matrix may affect the performance of the algorithm significantly. In particular, 
the number of data snapshots available may affect the estimate of $K$. As indicated in the previous section, accurate estimation of the rank of $S_{M}$ is essential for the modified MV method. It may be worthwhile to consider using an information theoretic measure such as Akaike Information Criterion (AIC) or the Minimum Description Length (MDL) criterion in estimating $K$ [43]. Future studies of the extended Ferrara/Parks method should also include a MUSIC formulation for comparison. Also, while this thesis is primarily concerned with power estimation (diagonal terms of $S_{M}$ ), the off-diagonal terms provide valuable information about the signal. In theory the extended version of the Ferrara/Parks algorithm generates estimates of these terms, but the accuracy of these estimates has not been investigated. 


\section{Chapter 6}

\section{Conclusion}

\subsection{Summary}

This thesis has examined the problem of estimating powers in the normal modes of an acoustic signal. The research focuses on array processing methods for the low order modes because they are the most useful at long ranges from the source. Vertical line arrays can resolve these modes based on differences in their spatial distributions.

Chapter 2 reviewed modal propagation in deterministic and random channnels and defined the mode estimation problem within a general framework. Four important issues in modal array processing were discussed: (i) ambient noise, (ii) modal orthogonality, (iii) data snapshots, and (iv) signal coherence.

Chapter 3 considered conventional mode estimation techniques. Most standard methods compute the mode amplitudes by minimizing a squared error criterion. Since least squares theory essentially ignores the noise components in the received signal, these methods perform poorly in low SNR environments. A numerical example demonstrated that bias errors can severely affect the accuracy of the LS estimator in high noise environments. The effects of mode orthogonality were studied, and it was shown that the degrees of freedom in the modeshape correlation matrix is a useful measure of the number of modes that can be estimated using least squares. Bias in the LS estimate is not affected by the number of data snapshots $(L)$, but the variance is a function of $\frac{1}{L}$. Signal coherence is not an issue for conventional estimators.

The poor performance of the least squares methods in noisy environments motivated the search for a new algorithm. Chapter 4 developed a new approach to the mode estimation 
problem based on a method proposed by Ferrara and Parks. Several examples showed that both Ferrara/Parks formulations, minimum variance and MUSIC, offer significant improvements over the least squares algorithm. In particular, it was shown that the new methods do not suffer the bias error that plagues the LS estimator at low SNR. Also the adaptive estimators appeared to be less sensitive to modal orthogonality than conventional methods. It was indicated that, after a correction, the MV estimator bias is independent of the number of data snapshots available. To first order, the MUSIC estimator bias does not depend on the number of snapshots either. The variances associated with MV and MUSIC are functions of $\frac{1}{L-N+1}$ and $\frac{1}{L}$, respectively.

Although the results for the new algorithms are quite favorable, they depend on the coherence of the received signal. It was shown that performance degrades significantly as signal coherence is lost. Chapter 5 presented an extension of the Ferrara/Parks method which addresses this problem. Two examples and a brief analysis indicated that the modified algorithm may have some advantages.

\subsection{Suggestions for Further Research}

This thesis has developed a new adaptive modal estimator and has demonstrated some of its adrantages, but much work remains to be done. First, it would be useful to obtain an analytical form for the eigenvalues and eigenvectors used in the Ferrara/Parks estimate for the case where the modes are not assumed to be orthogonal. Having analytical results might lend insight into the global localization problem exhibited by the MV estimator. In addition. it would allow for more accurate bias and variance predictions.

With respect to the estimation of random modes, the work presented in Chapter 5 is clearly preliminary. A thorough study of the modified Ferrara/Parks algorithm is necessary to determine whether it is a viable solution. The estimation the number of eigenvectors

in the modal signal subspace is a problem that has not been adequately addressed yet. In addition, it is important to weigh the added complexity of the Ferrara/Parks method against its advantages to see if this approach is practical.

Finally there is the issue of resolving multiple modal sources. Recall from Chapter 3 that estimating the power distribution for multiple sources is not possible with conventional techniques. The Ferrara/Parks adaptive estimator has the potential to solve this problem 
since multiple sources (with non-identical power distributions) should correspond to multiple peaks in the received power spectrum. This is an interesting area for research because of the potential applications for multiple source localization using MMP. 


\section{Appendix A}

\section{Fisher Information Matrix for}

\section{Modal Estimation}

The Cramer Rao bound expresses a limit on an estimator's error variance in terms of the inverse of the Fisher information matrix. The purpose of this appendix is to derive the Fisher information matrix $\mathbf{J}$ for the mode estimation problem. This discussion follows the developement by Baggeroer et. al. [45]. The elements of $J$ are defined as follows

$$
\mathbf{J}_{i j}=-\mathcal{E}\left\{\frac{\partial^{2}}{\partial g_{i} \partial g_{j}} \ln f_{\mathbf{r} \mid \mathbf{g}}(\mathbf{R} \mid \mathbf{G})\right\}
$$

where $\mathrm{g}$ is the vector of parameters to estimate, $\mathrm{r}$ is the vector of observations, and $f_{\mathbf{r} \mid \mathrm{g}}$ is the conditional probability density for the observations. Assuming that the observations are the output of a zero-mean Gaussian process with covariance $\mathrm{K}(\mathrm{g})$, Baggeroer et.al. determine the following expression for the elements of the Fisher information matrix, ${ }^{1}$

$$
\mathrm{J}_{i j}=\mathrm{TR}\left\{\mathrm{K}^{-1}(\mathrm{~g}) \frac{\partial \mathrm{K}(\mathrm{g})}{\partial g_{i}} \mathbf{K}^{-1}(\mathrm{~g}) \frac{\partial \mathrm{K}(\mathrm{g})}{\partial g_{j}}\right\}
$$

\footnotetext{
${ }^{1}$ Due to a misprint in the paper, Baggeroer has a minus sign in the formula, but Equation A.2 is correct.
} 
where TR is the matrix trace operator. For modal array processing the vector of observables contains $L$ data snapshots, partitioned as follows,

$$
\mathrm{r}=\left[\begin{array}{c}
\mathrm{p}_{1} \\
\mathrm{p}_{2} \\
\vdots \\
\mathrm{p}_{L}
\end{array}\right]
$$

Since the snapshots are independent, the covariance $\mathrm{K}$ is a block diagonal matrix,

$$
\mathrm{K}=\left[\begin{array}{lll}
\mathrm{S} & & 0 \\
& \ddots & \\
0 & & \mathrm{~S}
\end{array}\right]
$$

where $\mathrm{S}$ is the sensor covariance matrix. Thus Eq. A.2 becomes

$$
\mathbf{J}_{i j}=L \cdot \mathrm{TR}\left\{\mathrm{S}^{-1}(\mathrm{~g}) \frac{\partial \mathrm{S}(\mathrm{g})}{\partial g_{i}} \mathbf{S}^{-1}(\mathrm{~g}) \frac{\partial \mathrm{S}(\mathrm{t})}{\partial g_{j}}\right\} .
$$

The average mode powers are the desired parameters to estimate in this thesis. In other words the vector $\mathrm{g}$ contains the diagonal terms of the $\mathbf{S}_{\mathrm{M}}$ matrix. For coherent mode estimation $g_{i}=\mathcal{P}_{T} a_{T_{i}}^{2}$ and routine math leads to a relatively simple expression for the derivative matrix, e.g., for $g_{1}=\mathcal{P}_{T} a_{T_{1}}^{2}$

$$
\frac{\partial \mathrm{S}}{\partial g_{1}}=\mathcal{P}_{T} \mathbf{E}\left[\begin{array}{cccc}
1 & \frac{a_{T_{2}}}{2 a_{T_{1}}} & \cdots & \frac{a_{T_{1 V}}}{2 a_{T_{1}}} \\
\frac{a_{T_{2}}}{2 a_{T_{1}}} & 0 \cdots & 0 & \\
\vdots & & \ddots & \vdots \\
\frac{a_{T_{2}}}{2 a_{T_{1}}} & 0 \cdots & 0 &
\end{array}\right] \mathbf{E}^{\dagger}
$$




\section{Appendix B}

\section{Mean and Variance of the Least Squares Power Estimates}

The purpose of this appendix is to compute the mean and variance of the least squares modal power estimates. The discussion below assumes that the incident field consists of the modal signal plus white noise and that the number of modes estimated is greater than the highest mode present in the signal.

Consider the least squares estimate of the mode correlation matrix based on the sample covariance matrix $\hat{\mathrm{S}}$

$$
\widehat{\mathrm{S}}_{\mathrm{M} \_L S}=\left(\mathrm{E}^{\dagger} \mathrm{E}\right)^{-1} \mathrm{E}^{\dagger} \hat{\mathrm{S} E}\left(\mathrm{E}^{\dagger} \mathrm{E}\right)^{-1}
$$

Assuming zero-mean data, $\hat{\mathbf{S}}$ is an unbiased estimate of $\mathbf{S}$, therefore the expected value of the LS estimate is identical to the ideal covariance result. This leads to the following expression for the expected power in mode $j$ in terms of the true power $P_{j}$

$$
\mathcal{E}\left\{\widehat{P}_{j \_\mathrm{s}}\right\} \equiv \bar{P}_{j \_\mathrm{ls}}=P_{j}+\sigma_{w}^{2}\left[\left(\mathrm{E}^{\dagger} \mathrm{E}\right)^{-1}\right]_{j j} .
$$

The LS bias depends only on the white noise and the modeshape correlation; it is unaffected by the number of snapshots used to form $\hat{\mathrm{S}}$.

The statistics of the sample covariance matrix determine the variance of the least squares power estimate. According to Goodman [20], the matrix $L \widehat{\mathrm{S}}$ follows a Complex Wishart distribution of order $N$ with $L$ degrees of freedom, where $N$ is the number of sensors and $L$ is the number of snapshots. This is often abbreviated: $L \widehat{\mathrm{S}} \sim \mathrm{CW}(L, N ; \mathrm{S}) . \mathrm{S}$ is the ideal 
(true) $N \times N$ sensor covariance matrix. The variance derivation makes use of the following theorem, due to Muirhead. [21]

Theorem 1 If $\mathrm{B}$ is a $K \times N$ matrix with rank $K$ and $\mathrm{A}$ is $\mathrm{CW}(L, N ; \mathrm{S})$, then $\mathrm{C}=\mathrm{BAB}^{\dagger}$ is $\mathrm{CW}\left(L, K ; \mathrm{BSB}^{\dagger}\right)$.

Based on Theorem 1, $L \widehat{\mathrm{S}}_{\mathrm{M} \perp \mathrm{SS}}$ is $\mathrm{CW}\left(L, M ;\left(\mathrm{E}^{\dagger} \mathrm{E}\right)^{-1} \mathrm{E}^{\dagger} \mathrm{SE}\left(\mathrm{E}^{\dagger} \mathrm{E}\right)^{-1}\right)$, provided that $\mathrm{E}$ has $M$ linearly independent columns where $M$ is equal to number of modes estimated. Suppose that $\mathrm{B}_{j}$ is a $1 \times M$ vector with a one in the $j$ th column and zeros everywhere else e.g., $\mathbf{B}_{1}=\left[\begin{array}{llll}1 & 0 & \cdots & 0\end{array}\right]$. This leads to the distribution shown below

$$
L \widehat{P}_{j \_l s}=\mathbf{B}_{j}\left(L \widehat{\mathbf{S}}_{\mathrm{M} \_L \mathrm{~S}}\right) \mathbf{B}_{j}^{\dagger} \sim \mathrm{CW}\left(L, 1 ; \bar{P}_{j \_l s}\right) .
$$

Recall that $\bar{P}_{j \_s}$ is the ideal covariance estimate of the power in mode $j$. According to the definition of the Wishart distribution, $\mathrm{CW}\left(L, 1 ; \bar{P}_{j\lrcorner \mathrm{s}}\right)$ denotes a multiple of a complex chi-squared random variable. The PDF for a complex chi-squared random variable $y$ with $L$ degrees of freedom is given below

$$
f_{y}(Y)=\frac{Y^{L-1} e^{-Y}}{(L-1) !}
$$

Integration by parts reveals that both the mean and variance of $y$ are equal to $L$. The least squares estimate of the power in mode $j$ is a multiple of a chi-squared random variable

$$
\widehat{P}_{j\lrcorner \mathrm{s}}=\frac{\bar{P}_{j \_\mathrm{s}}}{L} \chi_{L}^{2}
$$

Therefore, the variance of the LS estimate becomes

$$
\operatorname{Var}\left\{\widehat{P}_{j \_\mathrm{s}}\right\}=\left(\frac{\bar{P}_{j \_\mathrm{s}}}{L}\right)^{2} \cdot L=\frac{\left(\bar{P}_{j \_\mathrm{s}}\right)^{2}}{L}
$$




\section{Appendix $\mathrm{C}$}

\section{Mean and Variance of the MV}

\section{Power Estimates}

This appendix contains the mean and variance calculations for the minimum variance modal power estimates. According to Chapter 4, the MV method uses estimates of the relative modal distribution and the associated signal power to form the mode correlation matrix

$$
\widehat{\mathrm{S}}_{\mathrm{M} \_\mathrm{MV}}=\widehat{\mathcal{P}}_{\mathrm{mv}} \widehat{\mathrm{a}}_{\mathrm{mv}} \widehat{\mathrm{a}}_{\mathrm{mv}}^{\dagger} \text {. }
$$

Determining an exact distribution for the modal powers (diagonal elements of $\widehat{\mathrm{S}}_{\mathrm{M} \_\mathrm{MV}}$ ) requires a thorough analysis of the optimization problem used in the Ferrara/Parks approach. In general, this is a difficult task because of the complicated interaction of noise, orthogonality, and snapshot issues. Fortunately, a simplifying assumption leads to a useful approximation for the distribution. The discussion below assumes that $\widehat{\mathrm{a}}_{\mathrm{mv}}$ corresponds to the true mode coefficient vector $\mathrm{a}_{\mathrm{T}}$, therefore any fluctuations in the estimate are due to $\widehat{\mathcal{P}}_{\text {mv }}$. Recall from Chapter 4 that this is a reasonable assumption provided that the sampled modeshapes are relatively orthogonal.

The statistics of the minimum variance power estimate are well-understood $[22,19]{ }^{1}$ According to Capon and Goodman [22], $\widehat{\mathcal{P}}_{\mathrm{mv}}$ is a multiple of a complex chi-squared random

\footnotetext{
${ }^{1}$ Steinhardt [19] provides an excellent review of this topic.
} 
variable with $L-N+1$ degrees of freedom, i.e.,

$$
\widehat{\mathcal{P}}_{\mathrm{mv}} \sim \mathcal{P}_{\mathrm{mv}}\left(\frac{1}{L}\right) \chi_{L-N+1}^{2}
$$

$L$ is the number of data snapshots used to estimate the covariance matrix, $\mathrm{S}$, and $N$ is the number of sensors in the array. $\mathcal{P}_{\mathrm{mv}}$ is the power estimate based on the ideal sensor covariance. See Appendix B for the probability density function of a complex chi-squared random variable. Based on the $\mathrm{PDF}$, the mean and variance of $\chi_{L-N+1}^{2}$ are both equal to $L-N+1$. As a result the MV power estimate is biased

$$
\mathcal{E}\left\{\widehat{\mathcal{P}}_{\mathrm{mv}}\right\}=\left(\frac{L-N+1}{L}\right) \mathcal{P}_{\mathrm{mv}}
$$

Although the estimate is asymptotically unbiased, the number of snapshots must be large in order to make the bias negligible. For this reason, some MV formulations correct the estimate by multiplying the $\widehat{\mathcal{P}}_{\mathrm{mv}}$ by $\frac{L}{L-N+1}$. The rest of this development and the results in Chapter 4 assume that this bias correction has been implemented.

Consider an incident field consisting of a modal signal plus white noise. The ideal MV power estimate is shown below

$$
\mathcal{P}_{\mathrm{mv}}=\mathcal{P}_{\mathrm{T}}+\frac{\sigma_{w}^{2}}{\beta}
$$

assuming that the mode correlation matrix is equal to $\beta \mathrm{I}$. This leads to an expression for the expected power in mode $j$

$$
\mathcal{E}\left\{\widehat{P}_{j_{-\mathrm{mv}}}\right\} \equiv \bar{P}_{j \_\mathrm{mv}}=P_{j}+\frac{\sigma_{w}^{2}}{\beta}\left[\mathrm{a}_{\mathrm{T}} \mathbf{a}_{\mathrm{T}}^{\dagger}\right]_{j j}
$$

where $P_{j}$ is the true power in mode $j$.

Accounting for the bias correction, the variance of the power estimate for mode $j$ becomes

$$
\operatorname{Var}\left\{\widehat{P}_{j \_\mathrm{mv}}\right\}=\frac{\left(\bar{P}_{j \_\mathrm{mv}}\right)^{2}}{L-N+1} .
$$




\section{Appendix D}

\section{Mean and Variance of the MUSIC}

\section{Power Estimates}

This appendix derives the mean and variance associated with the MUSIC modal power estimator shown below

$$
\widehat{\mathrm{S}}_{\text {M_MUSIC }}=\widehat{\mathcal{P}}_{\text {music }} \widehat{\mathrm{a}}_{\text {music }} \widehat{\mathrm{a}}_{\text {music }}^{\dagger} .
$$

As in Appendix C, a simplifying assumption facilitates these calculations. Specifically, the following development assumes that $\widehat{a}_{\text {music }}$ corresponds to the true modal distribution, $\mathbf{a}_{\mathrm{T}}$. This is equivalent to assuming that the algorithm locates the correct peak, thus any errors are due to the estimate of the signal power, $\widehat{\mathcal{P}}_{\text {music }}$. The assumption is reasonable, provided that the sampled modeshapes are approximately orthogonal.

The MUSIC power estimate is based on the maximum eigenvalue of the sample covariance matrix i.e.,

$$
\widehat{\mathcal{P}}_{\text {music }}=\frac{\widehat{\lambda}_{1}}{\widehat{\mathrm{a}}_{\text {music }}^{\dagger} \mathrm{E}^{\dagger} \mathrm{E} \hat{\mathrm{a}}_{\text {music }}} .
$$

The sample covariance matrix $\widehat{\mathrm{S}}$, formed from $L$ snapshots, follows a complex Wishart distribution: $\mathrm{CW}(L, N ; \mathrm{S})$. According to Kaveh and Wang [46] and references cited therein, the eigenvalues of a complex Wishart matrix with $L$ degrees of freedom have the mean and variance shown below

$$
\mathcal{E}\left\{\hat{\lambda}_{i}\right\}=\lambda_{i}+\mathcal{O}\left\{L^{-1}\right\}
$$


and

$$
\operatorname{Var}\left\{\widehat{\lambda}_{i}\right\}=\frac{\lambda_{i}^{2}}{L}+\mathcal{O}\left\{L^{-2}\right\}
$$

Ignoring the $\mathcal{O}\left\{L^{-1}\right\}$ term, the expected value of $\widehat{\mathcal{P}}_{\text {music }}$ is identical to the ideal covariance result derived in Equation 4.41. Thus, for a signal plus white noise, the expected power in mode $j$ is given below

$$
\mathcal{E}\left\{\widehat{P}_{j \text { music }}\right\} \equiv \bar{P}_{j \_ \text {music }}=P_{j}+\frac{\sigma_{w}^{2}}{\beta}\left[\mathrm{a}_{\mathrm{T}} \mathbf{a}_{\mathrm{T}}^{\dagger}\right]_{j j}
$$

where $P_{j}$ is the true power. The above expression implicitly assumes that the number of modes in the estimate exceeds the highest mode present in the signal.

Ignoring the $\mathcal{O}\left\{L^{-2}\right\}$ term, the variance expression reduces to a simple form, e.g., for mode $j$,

$$
\operatorname{Var}\left\{\widehat{P}_{j_{\text {_music }}}\right\}=\frac{\left(\bar{P}_{j \text { _music }}\right)^{2}}{L} .
$$




\section{Bibliography}

[1] C. Clay and H. Medwin, Acoustical Oceanography. New York: John Wiley and Sons, 1977.

[2] S. Jesus, "Normal-mode mathching localization in shallow water: Environmental and system effects," J. of the Acoustical Soc. of America, vol. 90, pp. 2034-2041, October 1991.

[3] E. Shang, "An efficient high-resolution method of source localization processing in mode space," J. of the Acoustical Soc. of America, vol. 86, pp. 1960-1967, November 1989.

[4] T. Yang, "A method of range and depth estimation by modal decomposition," J. of the Acoustical Soc. of America, vol. 82, pp. 1736-1745, November 1987.

[5] A. Baggeroer, W. Kuperman, and P. Mikhalevsky, "An overview of matched field methods in ocean acoustics," IEEE J. of Oceanic Engineering, vol. 18, pp. 401-424, October 1993.

[6] A. B. Baggeroer. Personal communications: Discussion of Heard Island Feasibility Test results.

[7] W. H. Munk, R. C. Spindel, A. B. Baggeroer, and T. G. Birdsall, "The Heard Island Feasibility Test." Submitted to J. Acoustical Soc. of America.

[8] A. Voronovich, V. Goncharov, A. Nikol'tsev, and Y. Chepurin, "Comparative analysis of methods for the normal mode decomposition of a sound field in a waveguide: numerical simulation and full-scale experiment," Soviet Physics. Acoustics, vol. 38, pp. 365-370, July-Aug. 1992.

[9] J. Polcari, Acoustic Mode Coherence in the Arctic Ocean. PhD thesis, Massachusetts Inst. of Technology/Woods Hole Oceanographic Inst., May 1986.

[10] L. Brekhovskikh and Y. Lysanov, Fundamentals of Ocean Acoustics. New York: Springer-Verlag, 1982.

[11] G. Frisk, Ocean and Seabed Acoustics. Englewood Cliffs, NJ: Prentice Hall, 1994.

[12] F. Jensen, W. Kuperman, M. Porter, and H. Schmidt, Computational Ocean Acoustics. New York, NY: American Institute of Physics Press, 1994.

[13] W. Munk, "Sound channel in an exponentially stratified ocean, with application to SOFAR," J. of the Acoustical Soc. of America, vol. 55, pp. 220-226, February 1974. 
[14] A. B. Baggeroer, "A numerical approach to the solution of acoustic wave equations." Unpublished.

[15] L. Dozier and F. Tappert, "Statistics of normal mode amplitudes in a random ocean. I. Theory," J. of the Acoustical Soc. of America, vol. 63, pp. 353-365, February 1978.

[16] L. Dozier and F. Tappert, "Statistics of normal mode amplitudes in a random ocean. II. Computations," J. of the Acoustical Soc. of America, vol. 64, pp. 533-547, August 1978.

[17] A. Baggeroer and W. Kuperman, "Stochastic matched field processing," J. of the Acoustical Soc. of America(Supl), vol. 91(s), May 1992.

[18] B. J. Sperry. Personal communications.

[19] A. Steinhardt, "Adaptive Multisensor Detection and Estimation," in Adaptive Radar Detection and Estimation (S. Haykin and A. Steinhardt, eds.), pp. 91-160, New York: John Wiley and Sons, 1992.

[20] N. Goodman, "Statistical analysis based on a certain multivariate complex Gaussian distribution," Ann. of Math. Stat., vol. 34, pp. 152-177, March 1963.

[21] R. J. Muirhead, Aspects of Multivariate Statistical Theory. New York, NY: John Wiley and Sons, 1982.

[22] J. Capon and N. Goodman, "Probability distributions for estimators of the frequencywavenumber spectrum," Proceedings of the IEEE, vol. 58, pp. 1785-1786, October 1970.

[23] C. Therrien, Discrete Random Signals and Statistical Signal Processing. Englewood Cliffs, NJ: Prentice-Hall, 1992.

[24] R. H. Ferris, "Comparison of Measured and Calculated Normal-Mode Amplitude Functions for Acoustics Waves in Shallow Water," J. of the Acoustical Soc. of America, vol. 52, no. 3, pp. 981-988, 1972.

[25] C. Clay, "Use of arrays for acoustics transmission in a noisy ocean," Review of Geophysics, vol. 4, no. 4, pp. 475-507, 1966.

[26] M. Hinich, "Maximum likelihood signal processing for a vertical array," J. of the Acoustical Soc. of America, vol. 54, no. 2, pp. 499-503, 1973.

[27] G. Wilson, R. Koch, and P.J.Vidmar, "Matched mode localization," J. of the Acoustical Soc. of America, vol. 84, pp. 310-320, July 1988.

[28] B. J. Sperry, "Modal analysis of vertical array receptions for the heard island feasiblity test," Master's thesis, Massachusetts Inst. of Technology/Woods Hole Oceanographic Inst., May 1994.

[29] C. Lawson and R. Hanson, Solving Least Squares Problems. Englewood Cliffs, NJ: Prentice-Hall, 1974.

[30] W. Menke, Geophysical Data Analysis: Discrete Inverse Theory. Orlando, FL: Academic Press, 1984. 
[31] K. Aki and P. Richards, Quantitative Seismology: Thoery and Methods, ch. 12. Freeman, 1980.

[32] S. Kay, Modern Spectral Estimation. Englewood Cliffs, NJ: Prentice Hall, 1988.

[33] S. Marple, Digital Spectral Analysis. Englewood Cliffs, NJ: Prentice Hall, 1987.

[34] D. Johnson and D. Dudgeon, Array Signal Processing. Englewood Cliffs, NJ: PrenticeHall, 1993.

[35] J. Capon, "High-resolution frequency-wavenumber spectrum analysis," Proc. IEEE, vol. 54, pp. 1400-1418, August 1969.

[36] R. Schmidt, Signal subspace approach to emitter location and spectral estimation. $\mathrm{PhD}$ thesis, Stanford Univ., November 1981.

[37] R. Roy and T. Kailath, "ESPRIT - Estimation of Signal Parameters Via Rotational Invariance Techniques," IEEE Trans. on ASSP, vol. 37, pp. 984-995, July 1989.

[38] E. Ferrara and T. Parks, "Direction finding with an array of antennas having diverse polarizations," IEEE Trans. on Antennas and Propagation, vol. AP-31, pp. 231-236, March 1983.

[39] G. Strang, Linear Algebra and Its Applications. Harcourt Brace Jovanovich, 3 ed., 1988.

[40] F. Gantmacher, Matrix Theory, vol. I. New York, NY: Chelsea Publishing Co., 1959.

[41] W. H. Press, S. A. Teukolsky, W. T. Vetterling, and B. P. Flannery, Numerical Recipes in Fortran: The Art of Scientific Computing. Cambridge University Press, 2 ed., 1992.

[42] V. Pisarenko, "The retrieval of harmonics from a covariance function," Geophysical Journal of Royal Astronomical Society, vol. 33, pp. 347-366, 1973.

[43] M. Wax and T. Kailath, "Detection of signals by information theoretic criteria," IEEE Trans. on ASSP, vol. ASSP-33, pp. 387-392. April 1985.

[44] M. Wax and I. Ziskind, "Detection of the Number of Coherent Signals by the MDL Principle," IEEE Trans. on ASSP, vol. 37, pp. 1190-1196, August 1989.

[45] A. Baggeroer, W. Kuperman, and H. Schmidt, "Matched field processing: Source localization in correlated noise as an optimum parameter estimation problem," J. of the Acoustical Soc. of America, vol. 83, pp. 571-587, February 1988.

[46] M. Kaveh and H. Wang, "Threshold Properties of Narrow-Band Signal-Subspace Array Processing Methods," in Advances In Spectrum Analysis and Array Processing, vol. 2, ch. 5, pp. 173-220, Englewood Cliffs, NJ: Prentice Hall, 1991. 